Verification of High Temperature Free Atom Thermal Scattering in MERCURY Compared to TART

D. E. Cullen, S. McKinley, C. Hagmann

November 27, 2006 
This document was prepared as an account of work sponsored by an agency of the United States Government. Neither the United States Government nor the University of California nor any of their employees, makes any warranty, express or implied, or assumes any legal liability or responsibility for the accuracy, completeness, or usefulness of any information, apparatus, product, or process disclosed, or represents that its use would not infringe privately owned rights. Reference herein to any specific commercial product, process, or service by trade name, trademark, manufacturer, or otherwise, does not necessarily constitute or imply its endorsement, recommendation, or favoring by the United States Government or the University of California. The views and opinions of authors expressed herein do not necessarily state or reflect those of the United States Government or the University of California, and shall not be used for advertising or product endorsement purposes.

This work was performed under the auspices of the U.S. Department of Energy by University of California, Lawrence Livermore National Laboratory under Contract W-7405-Eng-48. 
UCRL-TR-226340

\title{
Verification of High Temperature Free Atom Thermal Scattering in MERCURY Compared to TART
}

\author{
by \\ Dermott E. Cullen, LLNL \\ Scott McKinley, LLNL \\ Christian Hagmann, LLNL \\ Contact \\ Dermott E. Cullen \\ University of California \\ Lawrence Livermore National Laboratory \\ P.O.Box 808/L-159 \\ Livermore, CA 94550
}

Tele: 925-423-7359

E.Mail: cullen1@llnl.gov

Website: http://www.llnl.gov/cullen1

August 12006

\section{Overview}

This is part of a series of reports verifying the accuracy of the relatively new MERCURY [1] Monte Carlo particle transport code by comparing its results to those of the older TART [2] Monte Carlo particle transport code. In the future we hope to extend these comparisons to include deterministic (Sn) codes [3]. Here we verify the accuracy of the free atom thermal scattering model [4] by using it over a very large temperature range.

We would like to be able to use these Monte Carlo codes for astrophysical applications, where the temperature of the medium can be extremely high compared to the temperatures we normally encounter in our terrestrial applications [5]. The temperature is so high that is it often defined in $\mathrm{eV}$ rather than Kelvin. For a correspondence between the two scale 293.6 Kelvin (room temperature) corresponds to $0.0253 \mathrm{eV} \sim 1 / 40 \mathrm{eV}$. So that $1 \mathrm{eV}$ temperature is about 12,000 Kelvin, and $1 \mathrm{keV}$ temperature is about 12 million Kelvin.

Here we use a relatively small system measured in $\mathrm{cm}$, but by using $\rho R$ scaling [6] our results are equally applicable to systems measured in $\mathrm{Km}$ or thousands of $\mathrm{Km}$ or any size that we need for astrophysical applications. The emphasis here is not on modeling any 
given real system, but rather in verifying the accuracy of the free atom model to represent theoretical results over a large temperature range.

\section{Objectives}

There are two primary objectives of this report,

1) Verify agreement between MERCURY and TART results, both using continuous energy cross sections. In particular we want to verify the free atom scattering treatment in MERCURY as used over an extended temperature range; by comparison to many other codes for TART this has already been verified over many years $[4,7]$.

2) Demonstrate that this agreement depends on using continuous energy cross sections. To demonstrate this we also present TART using the Multi-Band method $[8,9]$, which accounts for resonance self-shielding, and Multi-Group method, without self-shielding [9].

\section{Conclusions}

Our conclusions are,

1) MERCURY and TART, both using the same continuous energy cross sections and physical models, are in excellent agreement. In particular the shift of the thermal Maxwellian produced by MERCURY is in excellent agreement with that produced by TART.

2) We still expect large differences for codes using approximate methods, such as multi-group. In particular for a deterministic code, e.g., Sn, even one using 616 groups, but not accounting for self-shielding, can expect large differences from the MERCURY and TART continuous energy results shown here - CAVEAT EMPTOR!!! 


\section{Definition of the Problem}

This report is related to earlier reports comparing code results for a theoretical cylindrical uranium pin centered in a surrounding square of water, modeled as infinitely long along the axis of the cylinder and infinitely repeating in the other two dimensions, i.e., an infinite lattice of pin-cells, similar to a water moderated, uranium fueled thermal reactor [4].

In the hope that we can obtain solutions from more computer codes, particularly deterministic (Sn) codes [3], here we have simplified the geometry even further, to be only one dimensional, spherically symmetric. For this study we have two concentric spheres,

The inner sphere $7.62 \mathrm{~cm}$ in radius The outer sphere $15.24 \mathrm{~cm}$ in radius

The inner sphere contains uranium: 75\% U235, 25\% U238 (atom ratios) at an overall density of 18.8 grams/cc.

The outer sphere contains water: 2 atoms of $\mathrm{H} 1$ to 1 atom of $\mathrm{O} 16$ at an overall density of 1 grams/cc.

The outer limit of the outer sphere is a non-return, leakage boundary. This geometry is the same for all temperatures; this is not realistic, but this is a completely theoretical problem, designed only to allow us to compare neutron transport code results.

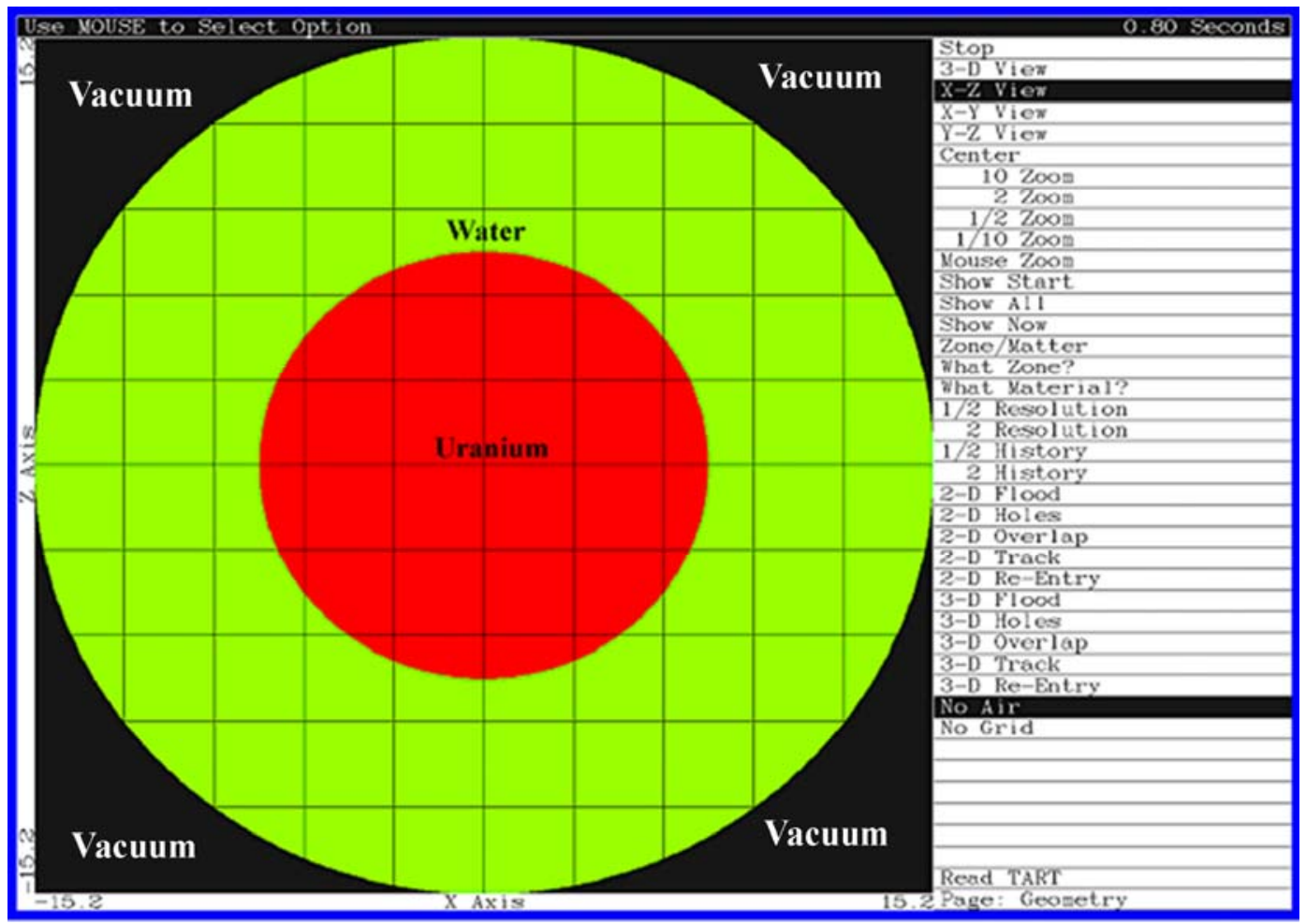


The dimensions and the composition were selected to make the room temperature (293.6 Kelvin) problem close to critical. Here we compare both integral parameters and differential spectra related to criticality. Specifically,

K-eff $=$ Production/[Absorption + Leakage $]$

In this equation production, absorption and leakage is each a single quantity integrated over the entire problem in space, energy and direction. For example, we can think of the production as first integrated over space and direction, and then written explicitly defining the integral over neutron energy, see Appendix $\mathrm{C}$ for details,

Production $=\int[v(E) \Sigma f(E)+2 \Sigma n, 2 n(E)+3 \Sigma n, 3 n(E) \ldots] \Phi(E) d E$

K-eff only depends on ratios of these quantities, therefore we normalize our results to one neutron produced, so that the other quantities are neutrons absorbed and leakage per neutron produced.

We first present the integral results: the single integral terms that appear in the definition of K-eff. We next present differential results: the energy dependent spectra that contribute to the integral results; here we present the flux, production, absorption and leakage spectra. It is important to understand that producing the same simple integral parameters, such as K-eff is necessary, but not sufficient to verify that codes actually agree in detail. By presenting detailed differential results we more strongly verify the agreement between our codes.

Both MERCURY and TART use continuous energy cross sections and the same physical models for these calculations. Both use the same TART 2005 nuclear data [10], which is based on ENDF/B-VI evaluations. To simplify comparisons all differential results use the TART 616 tally bins, which is 50 equally spaced bins per energy decade between $10^{-11}$ $\mathrm{MeV}$ and $20 \mathrm{MeV}$, i.e., equal lethargy intervals.

We are interested in results from other codes, particularly deterministic codes (Sn), and presumably these codes will use multi-group cross sections. Therefore in addition to the MERCURY and TART results using continuous energy cross sections, we also present TART results using the TART 616 group structure, using both multi-group and multiband methods.

By here comparing TART continuous, multi-band and multi-group results we can predict how well a deterministic code using multi-group cross sections can perform. Indeed given that deterministic codes use additional approximations, it is safe to say that we should expect the agreement between deterministic codes and our continuous energy Monte Carlo codes not to be any better than the results presented here comparing TART continuous energy and multi-group results. 


\section{Interpretation of Figures}

In this report we make extensive use of figures to compare results; here is a brief introduction to the format of these figures and how to interpret them. In each figure the upper $2 / 3$ present the data being compared, and the lower $1 / 3$ presents the ratio of all data to the first set of data, e.g., on the first plot the Ratio is the Mercury result divided by the TART result. Below we show two examples to illustrate why both data and ratio are needed.

In the first example the data agree so closely that it is difficult to tell there is more than one set of results shown, i.e., they agree to within the width of the line used to draw them. Based strictly on looking at the data they appear to exactly agree.

However, when we look at the ratio it looks like there are major differences between the two sets of data. Actually there are no major differences. Note the scale of the ratio is from 0.99 to 1.01 , in other words from $1 \%$ lower to $1 \%$ higher, with most of the differences being noise fluctuating near the $+/-0.1 \%$ level. For Monte Carlo code results we expect small random statistical differences such as this. In our calculations each code used $10^{8}$ source neutrons for each run. Spread over 616 groups we expect random differences near the $0.1 \%$ level, so that what we see here is acceptable, and does not indicate any significant difference between the results. Here the TART and MERCURY results are in excellent agreement.

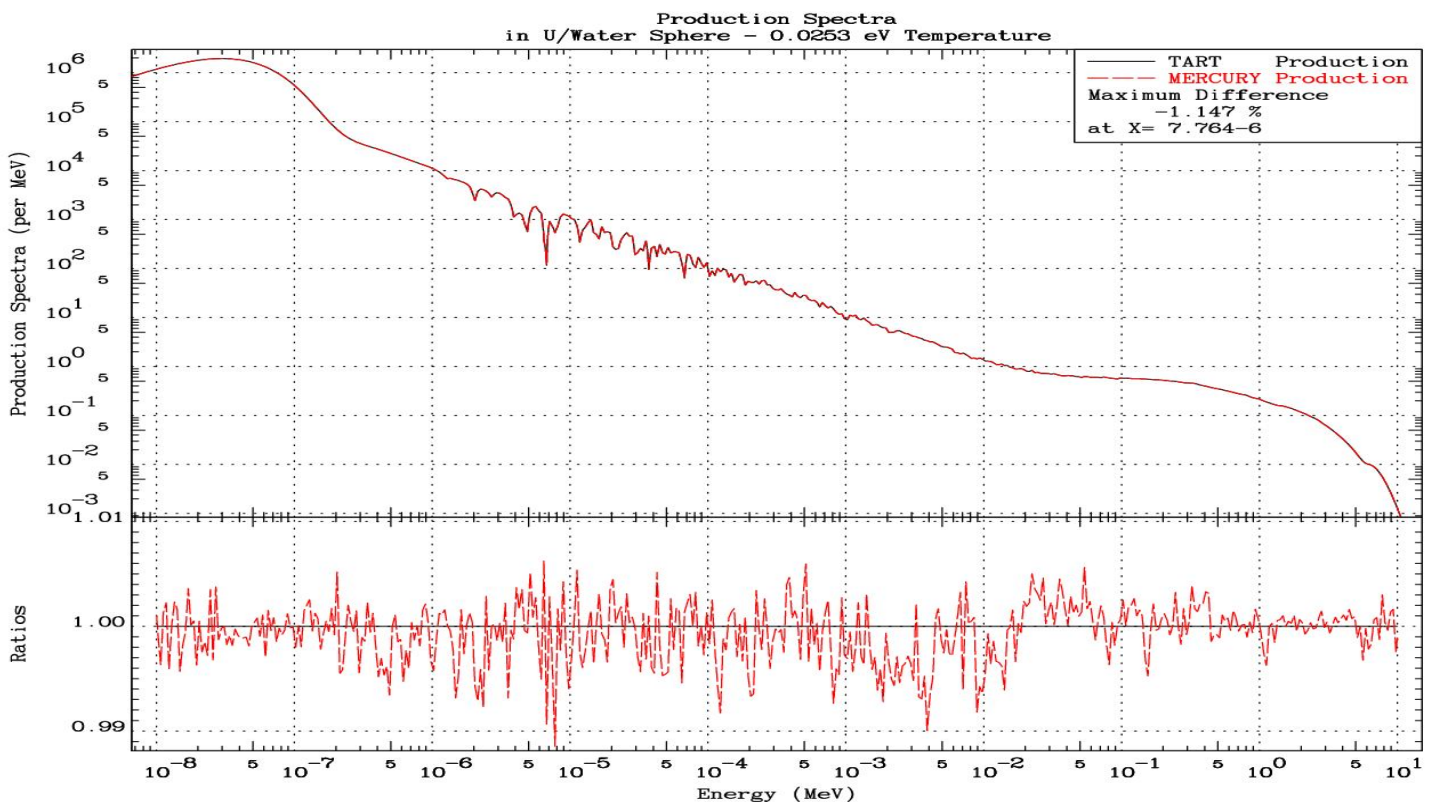


In the next example we use three TART results using various models. The data again agree so closely that it is difficult to tell there is more than one set of results shown, i.e., they agree to within the width of the line used to draw them. Based strictly on looking at the data they appear to exactly agree.

However, when we look at the ratio we now see real major differences between the three sets of data. Again, note the scale of the ratio which is now from about 0.96 to 1.01, in other words from $4 \%$ lower to $1 \%$ higher. Unlike the above first example where we only saw noise near the $+/-0.1 \%$ level, we now see real differences with the multi-group results differing from the continuous energy results over major portions of the energy range. This does indicate significant differences between the results. Here the multigroup results significantly differ from the continuous energy results. It is more difficult to see from the figure below that the multi-band results are in excellent agreement with the continuous energy results. The difference between multi-band and multi-group results are because the multi-band method accounts for self-shielding and the multi-group used here does not [9].

The bottom line in interpreting these figures is that you should consider both the data and the ratio to look for agreement - or disagreement,

1) We consider random noise near the $+/-0.1 \%$ level to be acceptable.

2) Larger differences, particularly where the difference lies above or below unity over an extend energy range, indicates unacceptable differences.

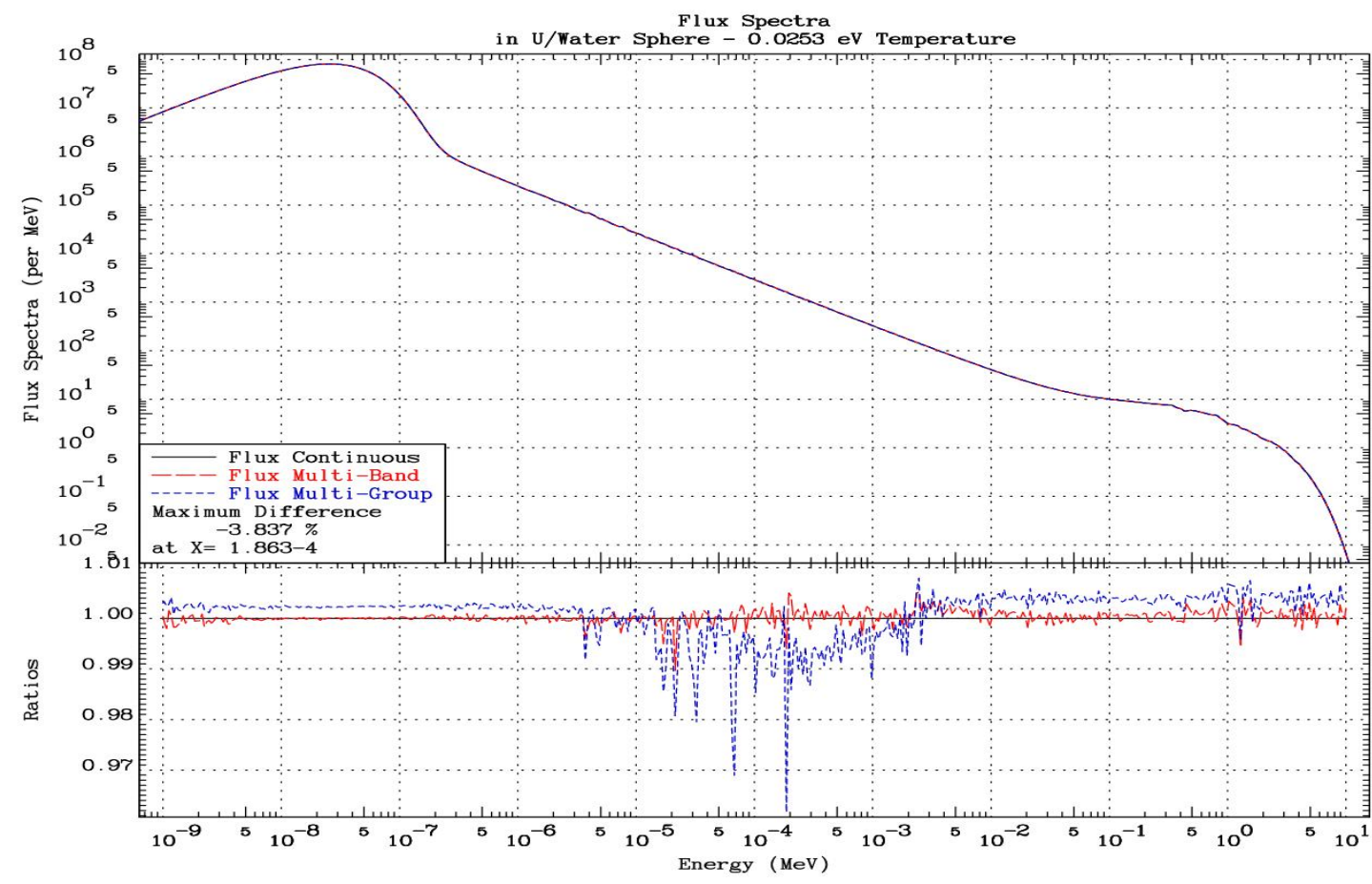




\section{The Effects of Temperature}

Temperature has a number of important effects on any neutron transport problems. Here we will only attempt to model some of these effects, namely those that we consider nuclear. For example, we will not model thermal expansion, i.e., the system is assumed to have exactly the same dimensions, density, etc., at all temperatures. All Doppler broadened cross section used in this study were produced using the standard SIGMA1 method $[9,11,12]$.

What we will attempt to model are three important effects: two effects on laboratory frame of reference cross sections, and one on the neutron spectrum. The effects are,

1) Doppler broadening of resonances: below is a comparison of $0.0253 \mathrm{eV}$ and $1 \mathrm{eV}$ uranium cross sections showing the Doppler broadening of the resonances with increasing temperature; resonances become smoother and wider.

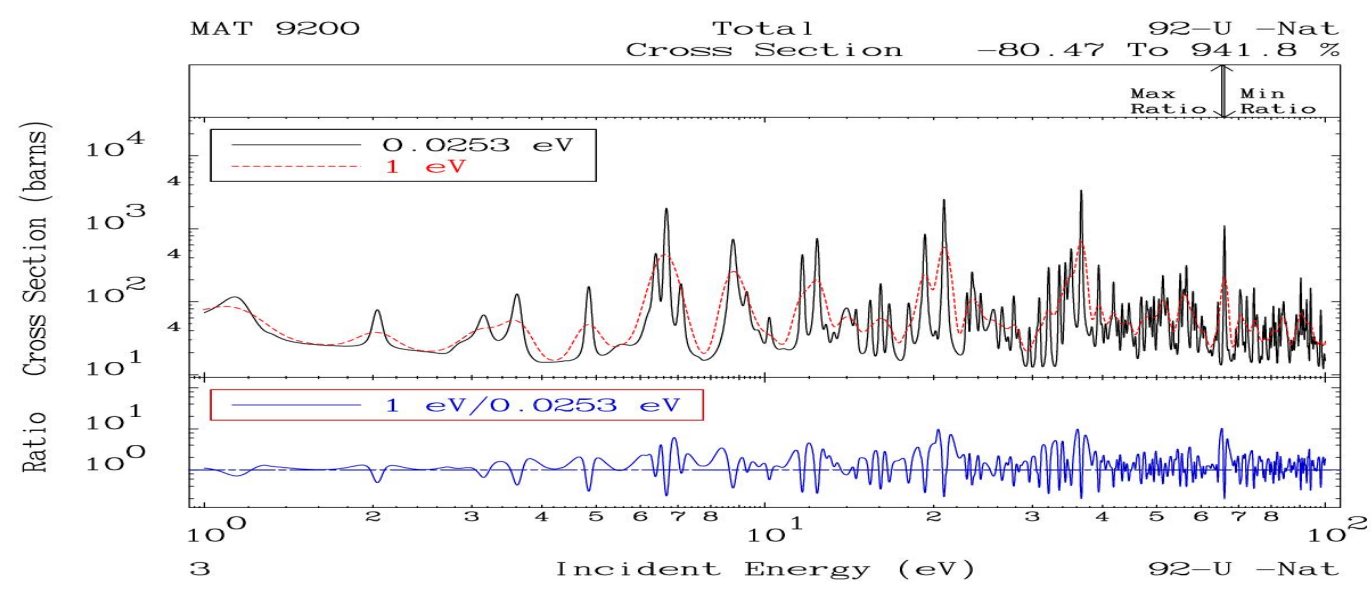

\section{Uranium Resonance cross sections Doppler broadening}

2) The often overlooked effect that temperature has on low energy elastic cross sections: below we see the effect that Doppler broadening has on the low energy hydrogen elastic cross section, causing the cross section at thermal energy to be $50 \%$ higher than its "cold" value of 20 barns.

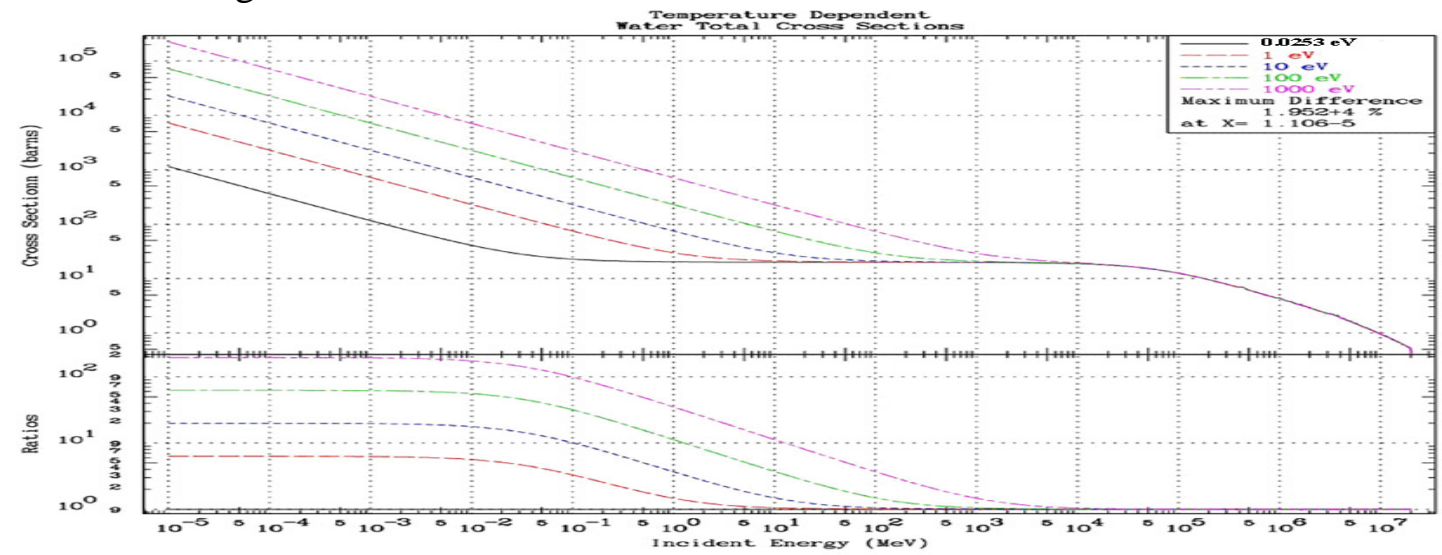

Hydrogen Elastic cross section Doppler broadening 
3) The most important effect is the shift in the thermal spectrum with energy. Even from the integral results we will see the dramatic effect that this has on parameters, such as the neutron removal time that varies by 3 orders of magnitude over the temperature range we consider: at $0.0253 \mathrm{eV}$ temperature (room) the neutron removal time is about $23.8 \mu$ second, whereas at $1000 \mathrm{eV}$ temperature it is only about $0.30 \mu$ second. What this means is that everything is happening a thousands times faster.

Below we show the neutron scalar flux integrated over the entire system at the five temperatures used in this study. Basically the fast fission neutrons slow down and approach thermal equilibrium at an energy defined by the local temperature. Naturally a neutron that reaches thermal equilibrium due to a $1 \mathrm{keV}$ temperature is moving much faster than one that thermalizes at room temperature. The temperature range here is from roughly $1 / 40 \mathrm{eV}$ to $1000 \mathrm{eV}$, a factor of 40,000 times different in neutron energy, or 200 times different in neutron speed. Since the neutrons move 200 times faster, no wonder everything is happening so much faster.

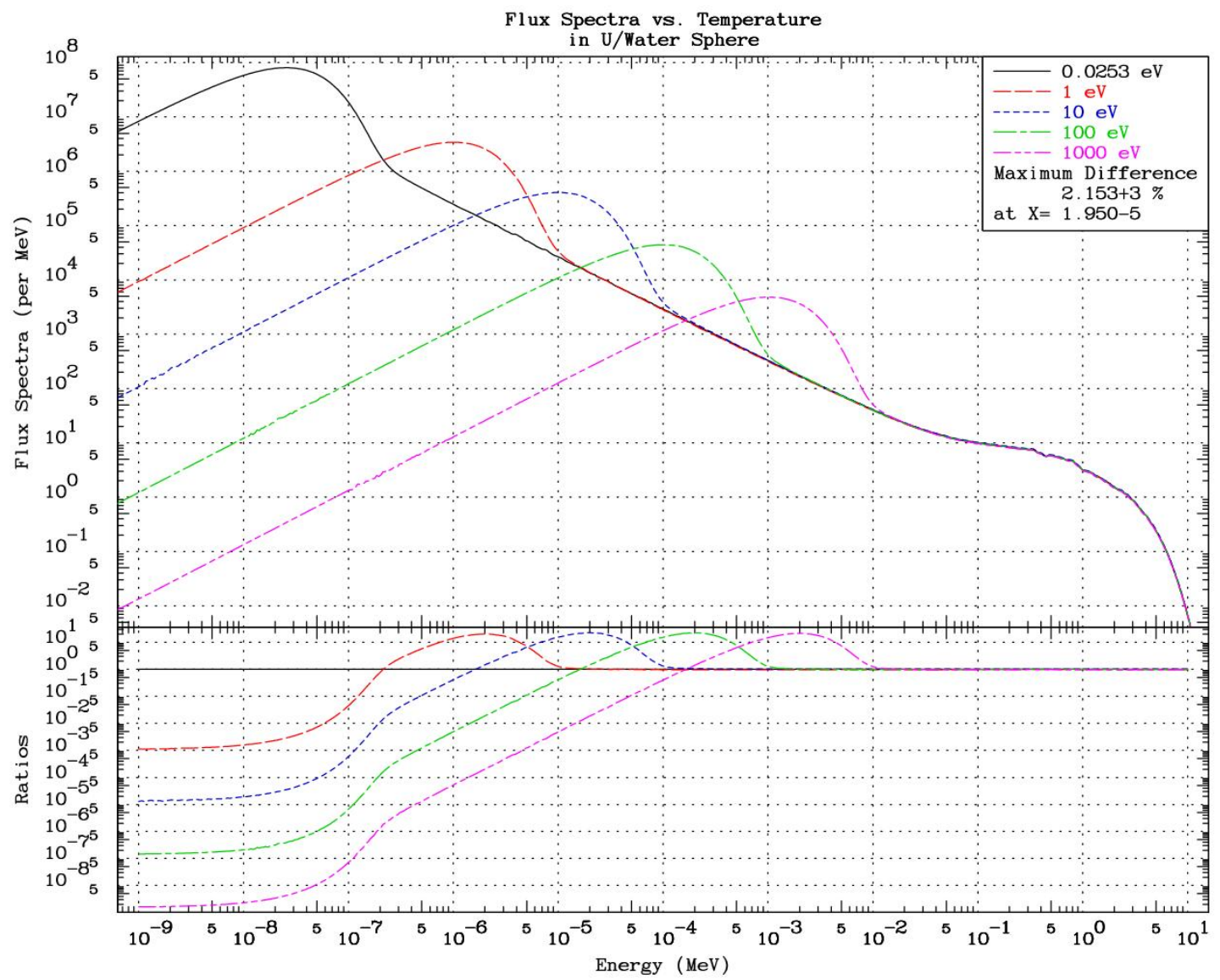

Flux Spectra vs. Temperature 


\section{Uranium Total Cross Section (75\% U235, 25\% U238)}

Below we show the uranium total cross sections at the five (5) temperatures used in this report [13]. In each figure we show the cross sections at two successive temperatures, to illustrate the change in cross section with temperature. Between $0.0253 \mathrm{eV}$ (room) and 1 $\mathrm{eV}$ there is an enormous smoothing of the cross section in the resonance energy range (about $1 \mathrm{eV}$ to $10 \mathrm{keV}$ ). At progressively higher temperatures the cross section is smoother, and by $1 \mathrm{keV}$ temperature the resonance region has disappeared and all we see is a broad bump across the resonance energy range. Note that above about $10 \mathrm{keV}$ there is virtually no change in the cross section for temperatures between $0.0253 \mathrm{eV}$ and $1 \mathrm{keV}$, so we expect little effect on higher energy neutrons.

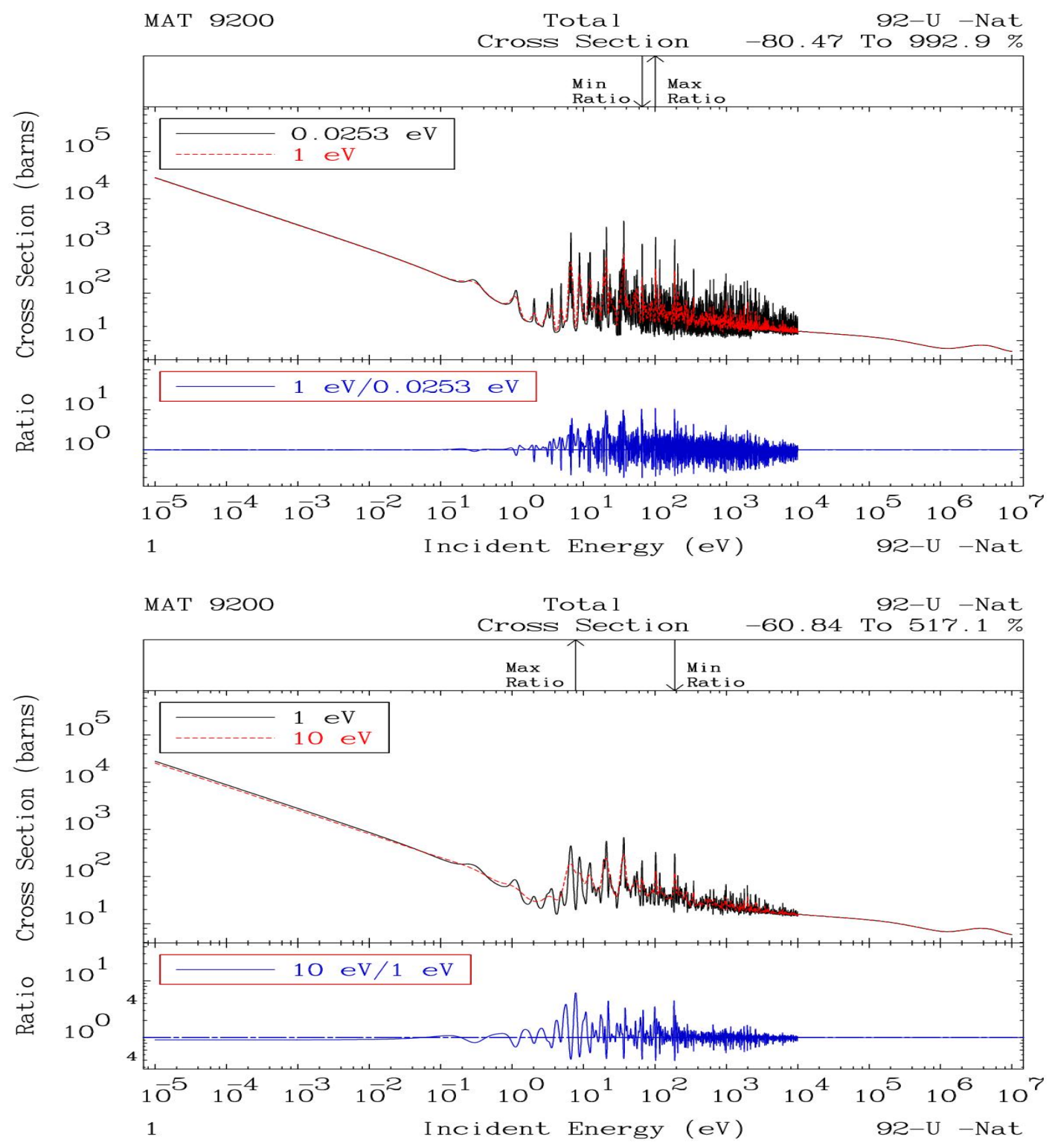




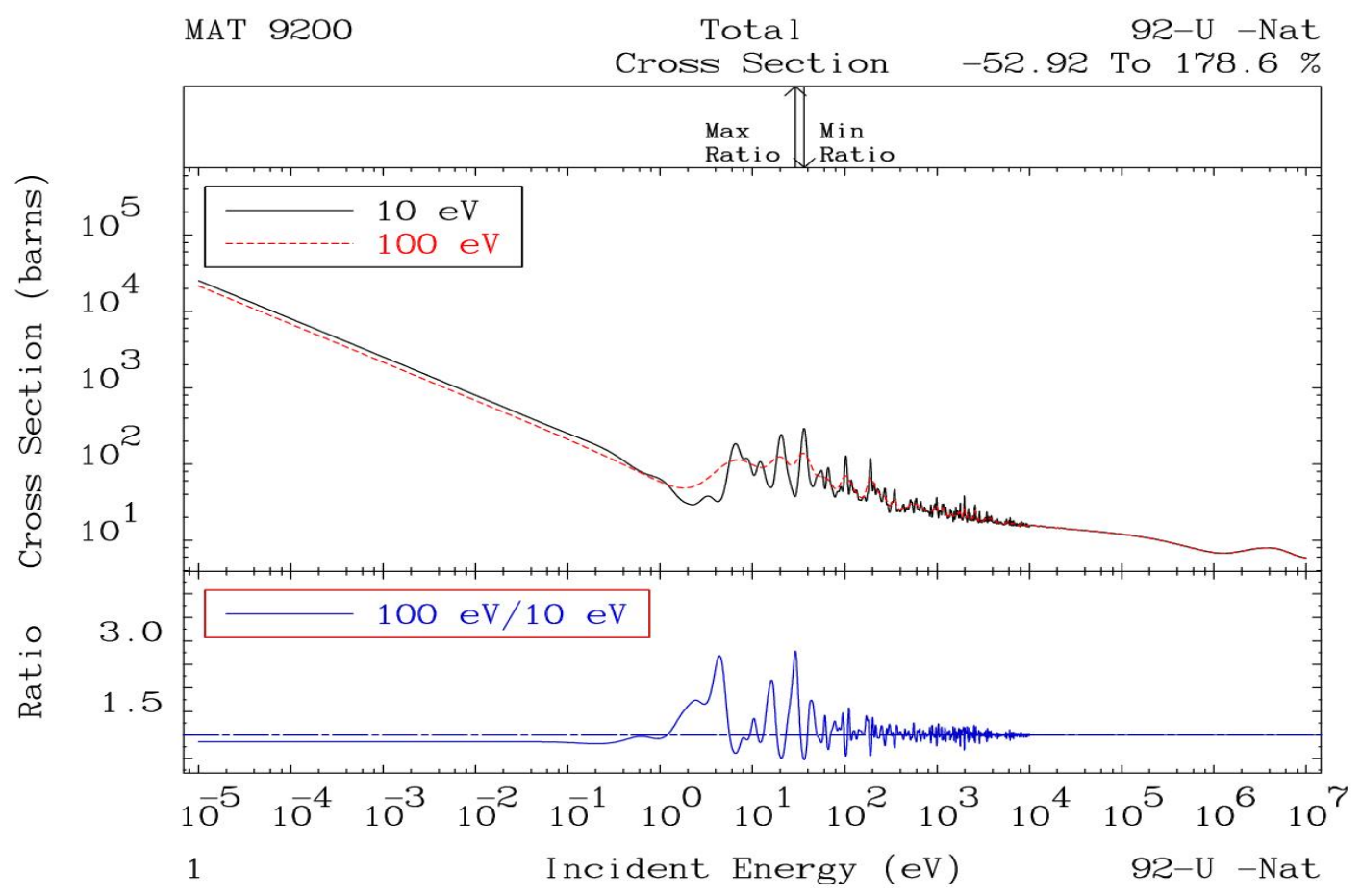




\section{Water Total Cross Section}

Below we show the water total cross sections at the five (5) temperatures used in this report [13]. A single figure shows the cross sections at all five temperatures. Basically what we see is Doppler broadening of the initially constant elastic scatter cross section. Doppler broadening "smoothes" the reaction rate $\mathrm{v} \sigma$, so that as this becomes "smoother" the low energy cross sections increase as $1 / \mathrm{v}$. This is an important effect in that the Doppler broadened hydrogen cross section at the energy corresponding to the temperature is roughly 50\% larger than the "cold" (0 Kelvin) cross section, i.e., 30 barns rather than 20 barns [this is true at all temperatures; at $1 \mathrm{eV}$ temperature the cross sections is 30 barns at $1 \mathrm{eV}$ incident neutron energy and at $1 \mathrm{keV}$ temperature the cross section is 30 barns at $1 \mathrm{keV}$ incident neutron energy]. The net effect as far as scattering is concerned is to make it appear that there is $50 \%$ more hydrogen present. Note that above about $10 \mathrm{keV}$ there is virtually no change in the cross section for temperatures between $0.0253 \mathrm{eV}$ and $1 \mathrm{keV}$, so we expect little effect on higher energy neutrons.

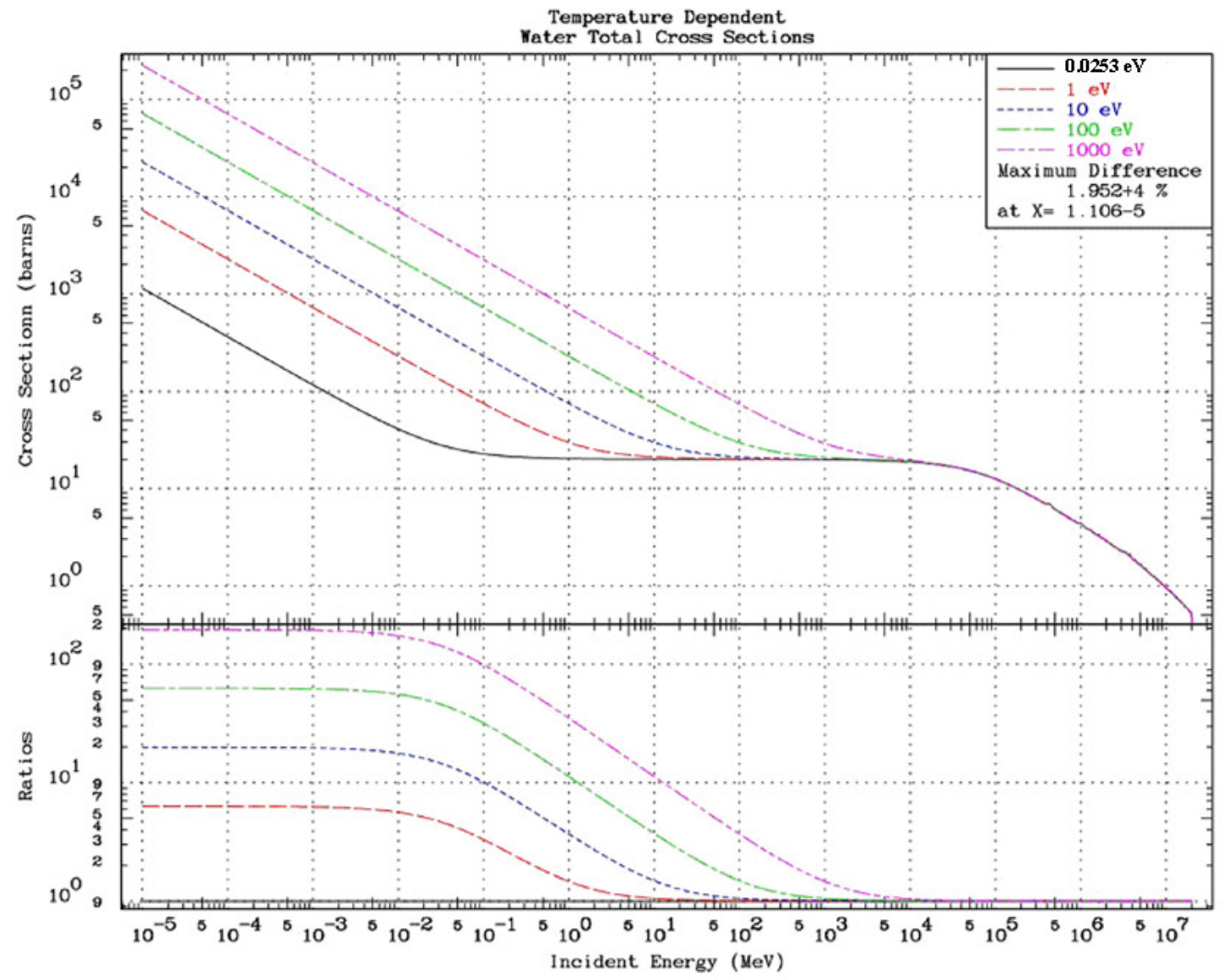




\section{What do we Expect for Results?}

Geometrically this is a very simple problem and we can qualitatively predict what we expect the answers to be. Below the upper figure shows the volume integrated flux in the uranium and water, and the lower figure shows the surface integrated current from uranium to water, water to uranium, and water to vacuum (the outside). The flux in the water is what we expect: the high energy fission emission, 1/E slowing down and Maxwellian at successively lower energies. The flux in the uranium differs from this because of the strong absorption during slowing down. The current spectra show the current from uranium to water driving the system and being dominant above about 30 $\mathrm{keV}$. Below this point slowing down in the water avoids absorption in the uranium, and allows the neutrons to survive down to lower energies where we can see the strong current from water back into the uranium, closing the cycle by causing fission in the uranium. From the current plot we can also see that at low energies not only is there a strong current flowing from water to uranium, but also a strong leakage current escaping from the system, i.e., this system is very leaky with about $40 \%$ of produced neutrons leaking.

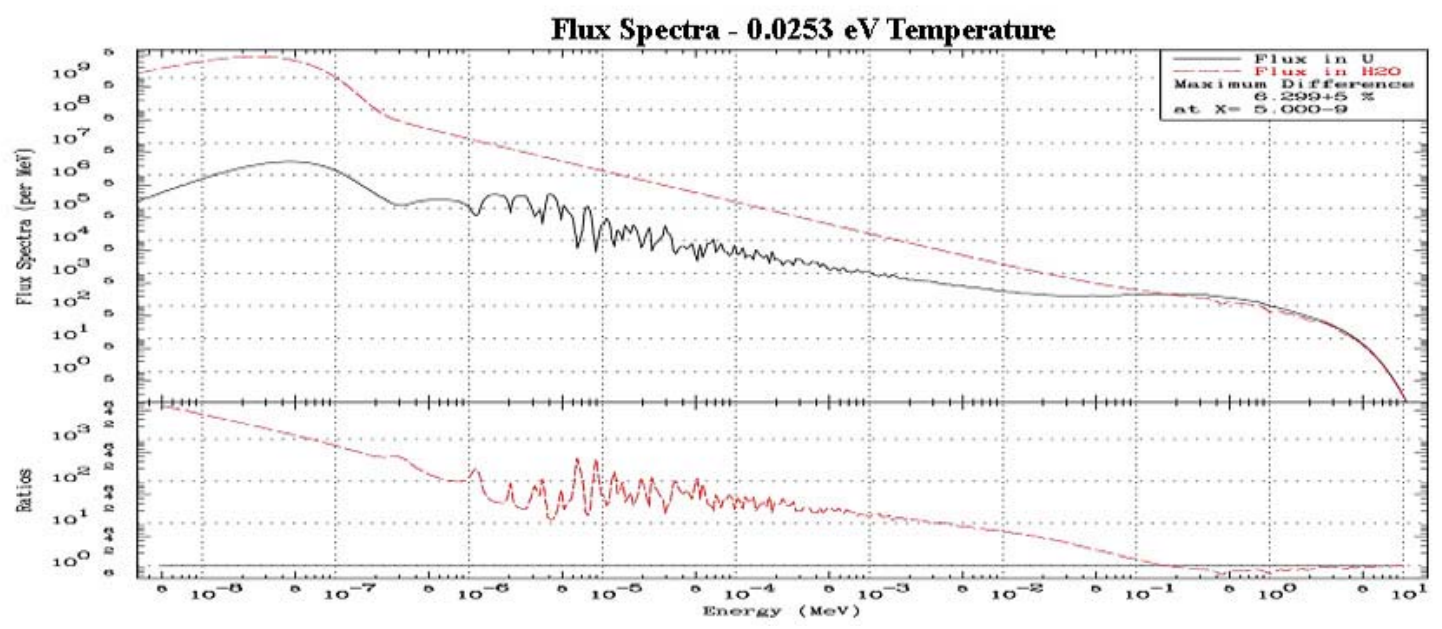

Flux Spectra

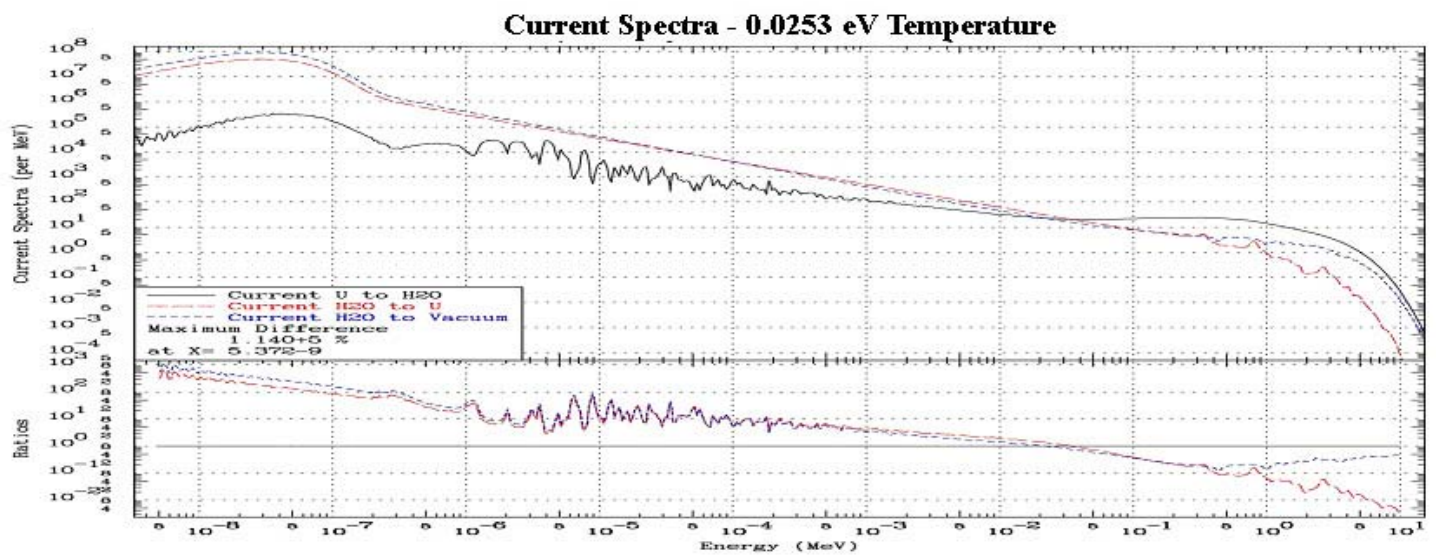

Current Spectra 
Besides the flux and current we can also look at the components that define K-eff, namely the spectra for production, absorption and leakage. Below we show these spectra in differential and integral form. The differential data are each normalized per neutron produced. The integral data are each normalized to unity when integrated over all energy.

Unlike a thermal reactor where production dominates only at high and low energy, here the high U235 (75\%) content makes production dominate over most of the energy range. The notable exceptions being near strongly absorbing resonances in the eV range and at very low energy, where absorption in water becomes dominant.

The above flux and current results and the below differential results make it appear that the spectra is dominated by a large thermal Maxwellian. However, from the below integral results we can see that most of the "action" (production, absorption and leakage) is actually happening at higher energies. For example, from the below integral plot we can see that the median fission energy (where the production integral crosses 0.5 ) is about $400 \mathrm{keV}$. This can be compared to a well thermalized reactor where most of the "action" is at thermal energies and the median fission energy is near $(3 / 2) \mathrm{KT} \sim 0.038 \mathrm{eV}$.

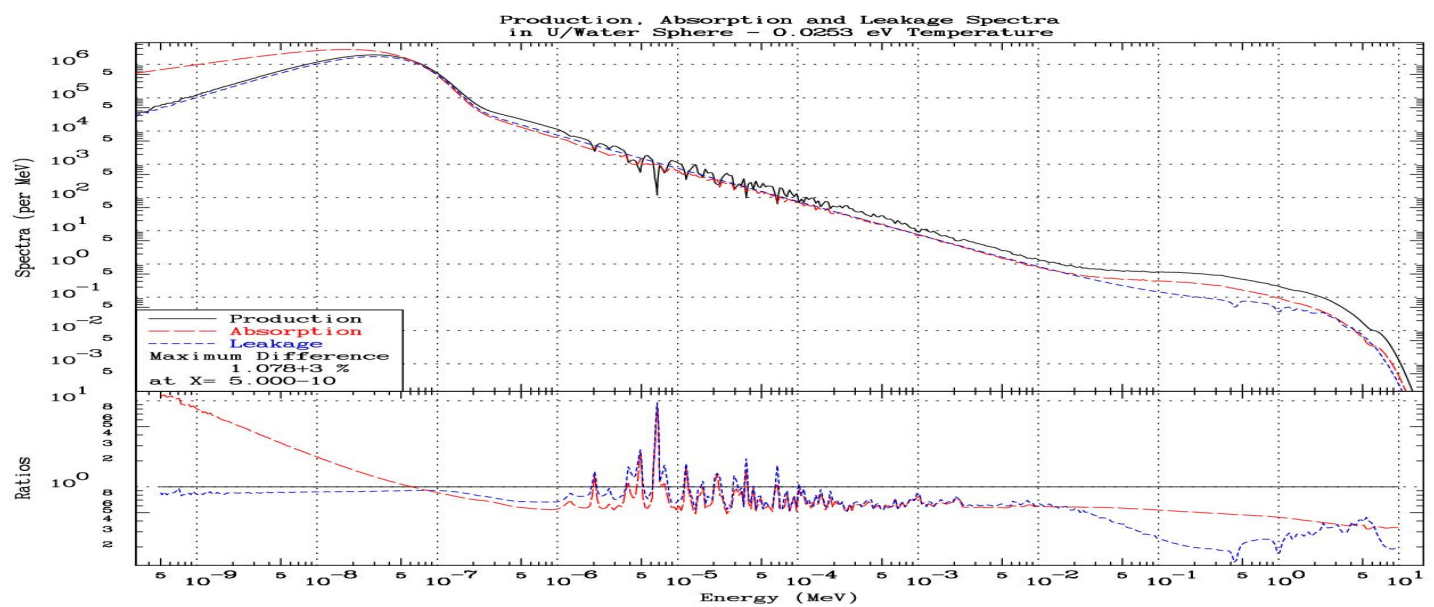

Differential Spectra

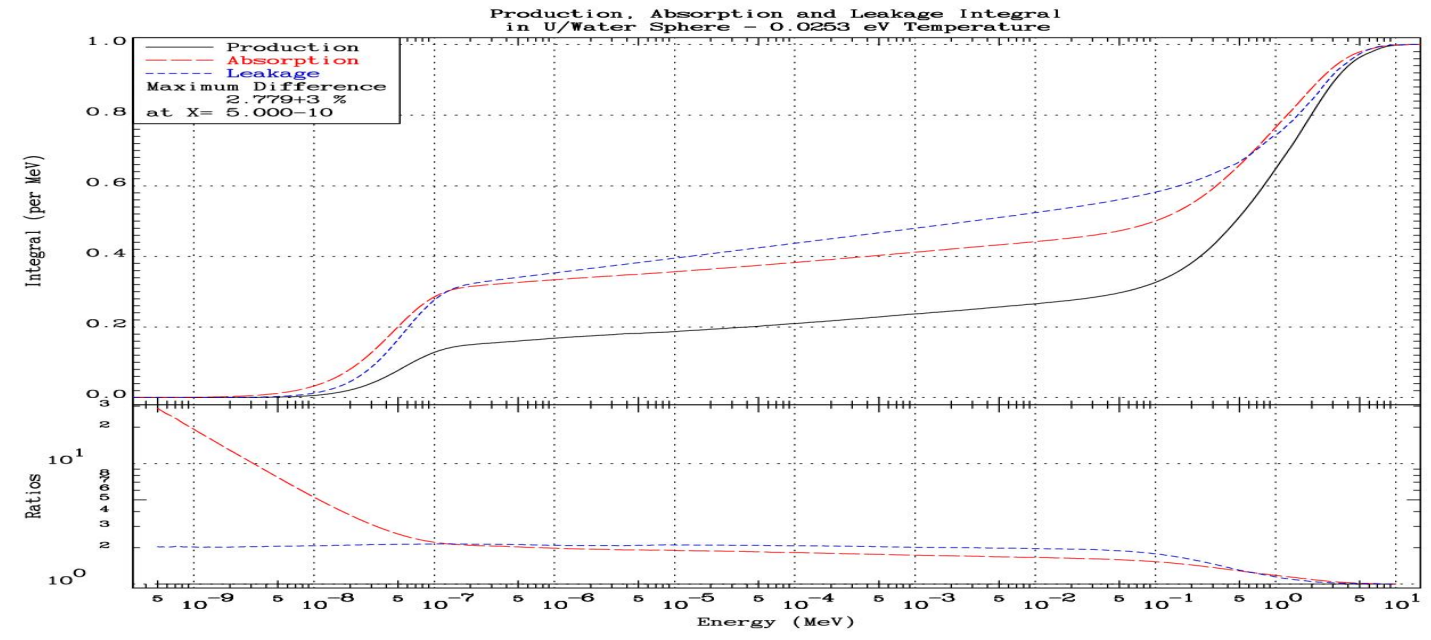

Integral Spectra 


\section{Continuous Energy v. Multi-Group}

MERCURY and TART can perform calculations using continuous energy cross sections or multi-group cross sections. The standard TART multi-group structure uses 616 energy groups, which is defined by 50 groups per energy decade between $10^{-11} \mathrm{MeV}$ to $20 \mathrm{MeV}$, i.e., equal lethargy intervals. You might think that 616 is a lot of groups and using continuous energy cross sections is overkill. This is not at all true. For these calculations the U238 cross sections use over 67,000 tabulated energy points. The 616 group TART structure does not come anywhere near accurately representing the energy dependence of the continuous energy cross sections. To illustrate this fact the figures below compare the continuous [13] and 616 group U238 cross sections [14]. The upper plot compares the 1 $\mathrm{eV}$ to $10 \mathrm{keV}$ energy range; these 4 decades of energy corresponds to 200 of the 616 groups. The lower plot compares the 1 to $2 \mathrm{keV}$ energy range; this energy range corresponds to about 16 groups. We can see the enormous difference between the continuous energy and multi-group cross sections; not small differences - ENORMOUS differences. Based on these differences unless the multi-group method accounts for selfshielding we should not be surprised to find large differences between continuous energy and 616 group results.
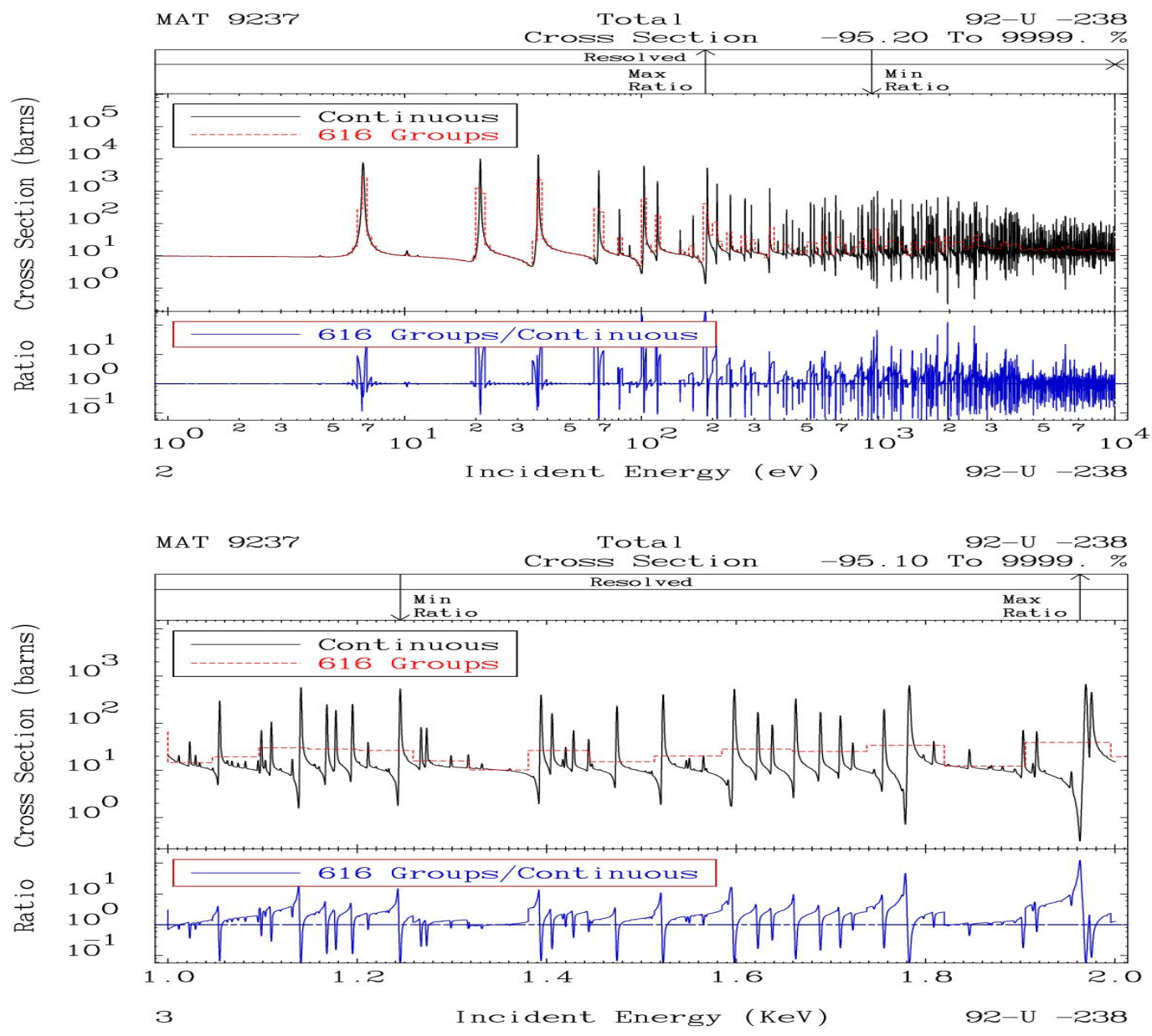


\section{Integral Results}

How accurate do we expect the results to be? In earlier studies comparing results from a variety of neutron transport codes each using whatever neutron data it felt to be appropriate, we found a spread in even the simple integral parameters, such as K-eff, in excess of $1 \%$ [4]. In earlier comparisons of MERCURY and TART we found that by having both codes use the same neutron data, namely TART's data, the differences in Keff could be reduced to less than $0.1 \%$ [7], indicating that most of the differences between codes is due to differences in nuclear data. In this study both codes are again using the same TART data and same physics models, so we expect results to be at the same $0.1 \%$ or better level.

In the following tables we should distinguish between qualities that are directly calculated and those that are derived from other quantities. For example, production, absorption, leakage, and removal lifetime are directly calculated (all then normalized per neutron produced), whereas K-eff and the system time constant $(\alpha)$ are derived as follows; see the Appendices for details,

K-eff $=$ Production $/$ Removal $=$ Production/[Absorption + Leakage $]$

For K-eff all of the quantities defining the ratio are positive and no differences are involved, so we expect the derived quantity, K-eff, to be as accurate as the individual quantities.

$$
\begin{aligned}
\alpha & =[\text { Production Rate }]-[\text { Removal Rate }]=\left[\frac{\text { Pr oduction }}{\text { Re moval }}-1\right] *[\text { Removal Rate }] \\
& =[\text { K-eff }-1] / \operatorname{Tr} \quad \operatorname{Tr}=\text { Removal Time }=1 /[\text { Removal Rate }]
\end{aligned}
$$

For $\alpha$ we have the difference [K-eff - 1], so we expect less accuracy, particularly when ever K-eff is close to unity. 
The tables below show what we expect, namely excellent agreement between MERCURY and TART, with differences in the directly calculated quantities and K-eff only in the fourth digit, i.e., less than $0.1 \%$. However, because in all cases K-eff is close to unity, differences in $\alpha$ are larger. In summary, in all cases we see excellent agreement between MERCURY and TART integral results. Therefore from the viewpoint of integral parameters these results verify the temperature dependent free atom scattering model used in MERCURY.

TART Continuous Energy Results

\begin{tabular}{|c|c|c|c|c|c|}
\hline $\begin{array}{c}\text { Temperature } \\
(\mathrm{eV})\end{array}$ & Absorption & Leakage & K-eff & $\begin{array}{c}\text { Removal Time } \\
(\mu \text { sec })\end{array}$ & $\begin{array}{c}\text { Alpha } \\
(1 / \text { usec })\end{array}$ \\
\hline $0.0253 \mathrm{eV}$ & $\mathbf{0 . 5 8 2 7 2}$ & $\mathbf{0 . 4 1 4 1 8}$ & 1.00311 & 23.767 & $1.3081 \mathrm{~d}-4$ \\
\hline $1 \mathrm{eV}$ & 0.51703 & 0.45835 & 1.02528 & 6.3769 & $3.9638 \mathrm{~d}-3$ \\
\hline $10 \mathrm{eV}$ & 0.53127 & 0.48648 & 0.98255 & 2.3003 & $-7.5832 \mathrm{~d}-3$ \\
\hline $100 \mathrm{eV}$ & 0.50891 & 0.47603 & 1.01523 & $\mathbf{0 . 8 2 7 4 7}$ & $1.8481 \mathrm{~d}-2$ \\
\hline $1000 \mathrm{eV}$ & $\mathbf{0 . 4 9 3 6 8}$ & $\mathbf{0 . 4 6 1 3 7}$ & 1.04707 & $\mathbf{0 . 2 9 9 5 3}$ & $\mathbf{1 . 5 7 1 4 d - 1}$ \\
\hline
\end{tabular}

MERCURY Continuous Energy Results

Red indicates difference from TART Continuous Energy results

\begin{tabular}{|c|c|c|c|c|c|}
\hline $\begin{array}{c}\text { Temperature } \\
(\mathbf{e V})\end{array}$ & Absorption & Leakage & K-eff & $\begin{array}{c}\text { Removal Time } \\
(\mu \text { sec })\end{array}$ & $\begin{array}{c}\text { Alpha } \\
(1 / \text { usec })\end{array}$ \\
\hline $0.0253 \mathrm{eV}$ & $\mathbf{0 . 5 8 2 6 5}$ & $\mathbf{0 . 4 1 4 5 1}$ & 1.00285 & 23.752 & $1.2016 \mathrm{~d}-4$ \\
\hline $1 \mathrm{eV}$ & $\mathbf{0 . 5 1 7 0 1}$ & $\mathbf{0 . 4 5 8 2 6}$ & 1.02536 & $\mathbf{6 . 3 7 6 3}$ & $3.9776 \mathrm{~d}-3$ \\
\hline $10 \mathrm{eV}$ & $\mathbf{0 . 5 3 1 2 4}$ & $\mathbf{0 . 4 8 6 5 5}$ & $\mathbf{0 . 9 8 2 5 3}$ & 2.2982 & $-7.6029 \mathrm{~d}-3$ \\
\hline $100 \mathrm{eV}$ & $\mathbf{0 . 5 0 8 8 8}$ & $\mathbf{0 . 4 7 6 1 0}$ & 1.01524 & $\mathbf{0 . 8 2 7 1 0}$ & $\mathbf{1 . 8 4 2 4 d - 2}$ \\
\hline $1000 \mathrm{eV}$ & $\mathbf{0 . 4 9 3 6 4}$ & $\mathbf{0 . 4 6 1 4 5}$ & 1.04701 & $\mathbf{0 . 2 9 9 3 7}$ & $\mathbf{1 . 5 7 0 4 d - 1}$ \\
\hline
\end{tabular}




\section{Why Agreement in K-eff is not enough}

In an earlier study we found good agreement between the K-eff values produced by TART and MCNP [4], but based on comparing spectra we can see that this is not good enough to guarantee that both codes are using the best physics. Below we show a detailed comparison of the neutron production spectra for the $1 / 2$ " radius pin cell as calculated by TART and MCNP. In the left figure we see excellent agreement over most of the energy range, except for at very low energy. The right figure shows a detail of the thermal energy range across what we expect to be a Maxwellisan-like thermal spectrum. From this figure we can see that TART is modelling the thermal scattering law as it is defined in ENDF/B-VI as continuous in secondary energy, whereas MCNP is modelling it as a series of discrete secondary energies for hydrogen bound in water, that does not look anything like the expected Maxwellian-like spectrum. The only reason that the MCNP spectrum does not drop even lower between the discrete energies, is because of free atom scattering in oxygen and to a lesser degree uranium.

The MCNP spectra shown below is not due to a code error; the code designers say this is what they intend, i.e., they admit that they are not using the continuous ENDF/B-VI thermal scattering law data, but rather a discrete approximation to it, and they admit that this approximation yields completely unphysical energy dependent spectra results, as shown below. Obviously we cannot use these unrealistic MCNP results for verification. MCNP results for ALL ENDF/B-VI thermal scattering law data show the same unrealistic "spikes", and are now available on-line at, http://www.llnl.gov/cullen1/therm2.htm
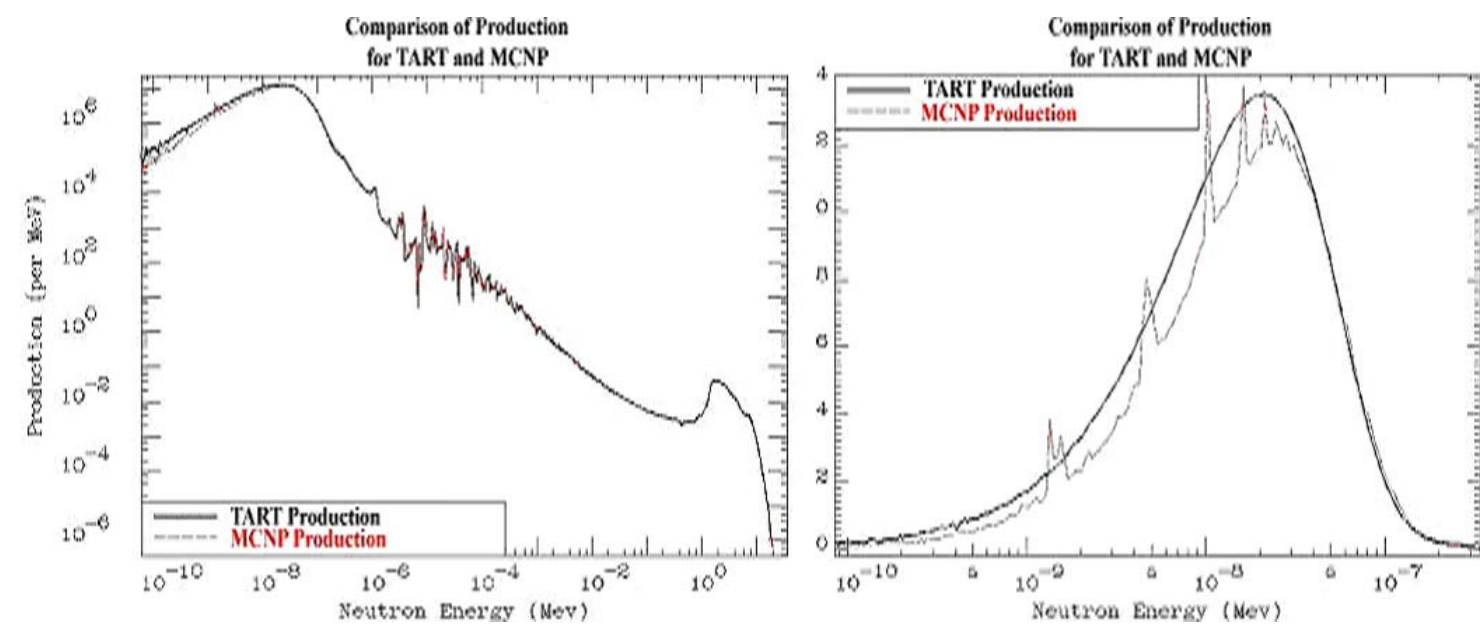

The bottom line is that today when we require highly accurate results, it is not good enough to merely insure that codes produce the same integral parameters; that is necessary, but not sufficient. Today we can, and should, go the extra step to insure that code differential results also agree. Therefore below we show comparisons of MERCURY and TART differential results. 


\section{Differential Comparisons}

Below we show comparisons of MERCURY and TART results at the five (5) temperatures included in this study [13]. At each temperature we show four figures, each figure comparing one type of spectra: flux, production, absorption, or leakage.

Interpretation of these figures does not require much comment. It is sufficient to point out that in ALL cases the scale of ratio (lower $1 / 3$ of each plot) is 0.99 to 1.01 , a narrow +/$1 \%$ band about unity. Based on the data (upper $2 / 3$ of each plot) the results agree to within the thickness of the lines, and the ratio (lower $1 / 3$ of each plot) show acceptable noise near the $0.1 \%$ level. In summary, in all cases we see excellent agreement between MERCURY and TART differential results. Therefore from the viewpoint of differential spectra these results verify the temperature dependent free atom scattering model used in MERCURY.

In terms of verifying the free atom scattering model used by MERCURY, what this model predicts is a neutron density (not flux) in a Maxwellian shape,

\section{$\mathrm{N}(\mathrm{E}) \mathrm{dE} \sim \sqrt{E} \quad \operatorname{Exp}[-\mathrm{E} / \mathrm{KT}] \mathrm{dE}$}

The flux is merely the neutron density times its speed, so we expect,

$\Phi(E) d E=\mathrm{v} N(E) \mathrm{dE} \sim \sqrt{E} \mathrm{~N}(\mathrm{E}) \mathrm{dE}$

$\Phi(E) d E=\mathrm{E} \operatorname{Exp}[-\mathrm{E} / \mathrm{KT}] \mathrm{dE}$

In ALL cases this is exactly the energy dependent shape of the flux produced by MERCURY and TART. The production, absorption, and leakage can then be predicted, for example, the absorption is: Absorption $=\Sigma a(E) \Phi(E) d E$

However, a word of caution: ALL of the spectra shown below are integrated over the entire system, i.e., both uranium and water spheres. The flux shown is dominated by the large volume and flux in the water, whereas above the thermal energy range the production and absorption spectra are defined solely upon what is happening in the uranium, e.g., there is no production in the water, and only very low energy absorption in the water. So that the spectra should be more clearly written to indicate spatial dependence, R: Absorption $=\Sigma a(E, R) \Phi(E, R) d E$

Because of this spatial dependence do not expect to see a clear correspondence between the system integrated flux and the production, absorption, and leakage spectra shown below.

With the above as background, below we present the MERCURY to TART comparison results without further comment. 


\section{MERCURY vs. TART: Thermal $(0.0253 \mathrm{eV})$ Temperature}

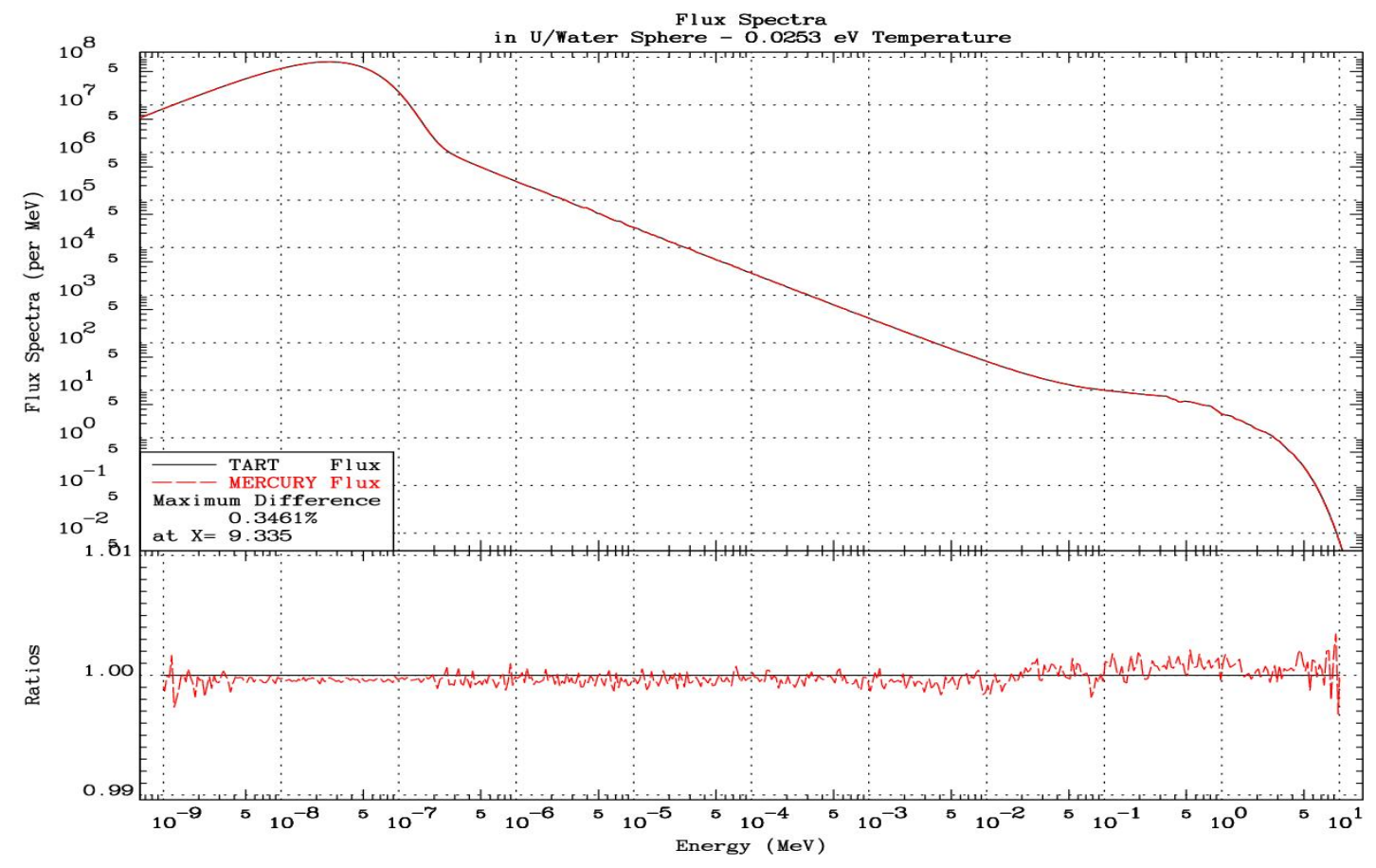

Flux Spectra

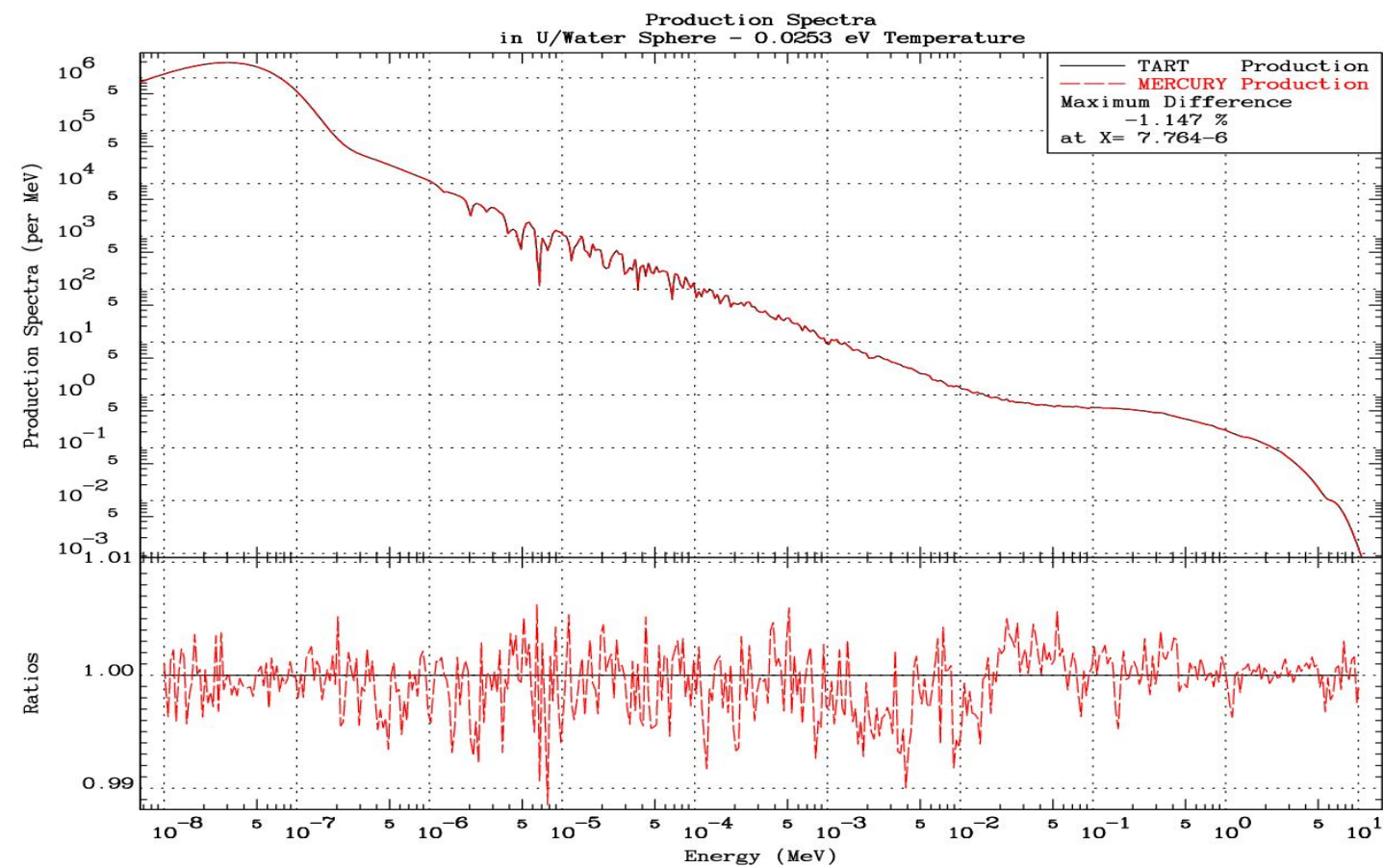

Production Spectra 


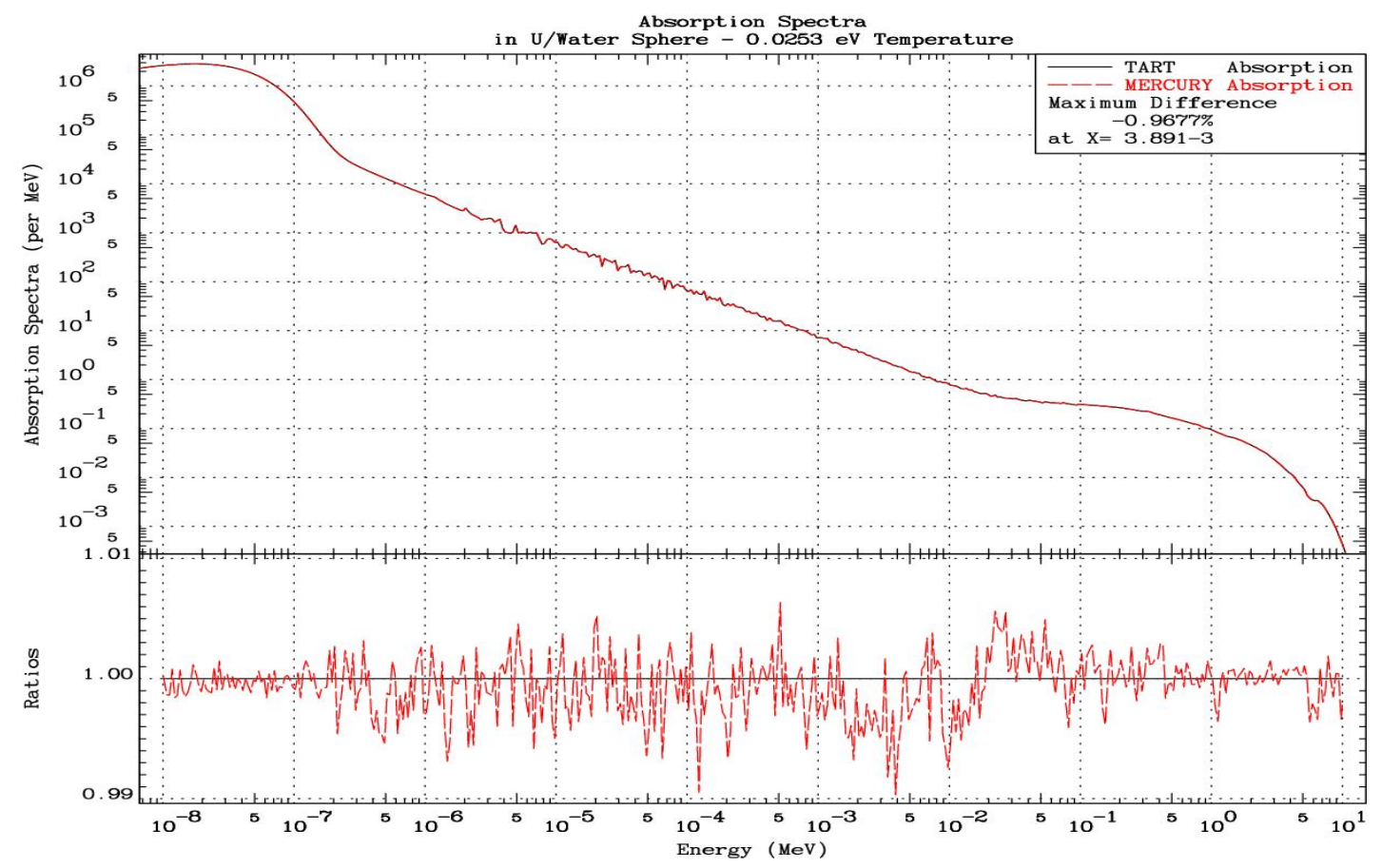

Absorption Spectra

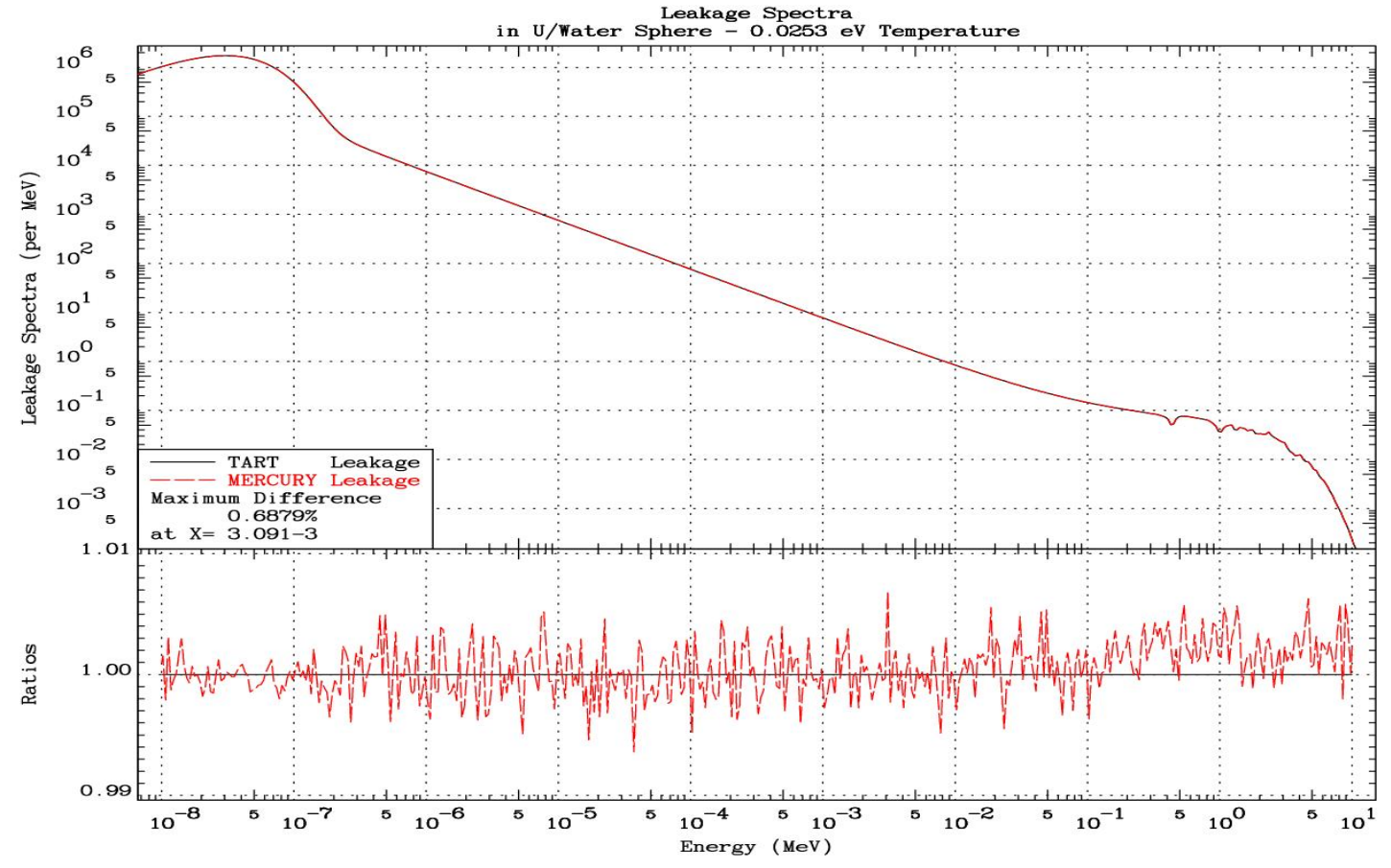

Leakage Spectra 


\section{MERCURY vs. TART: 1 eV Temperature}

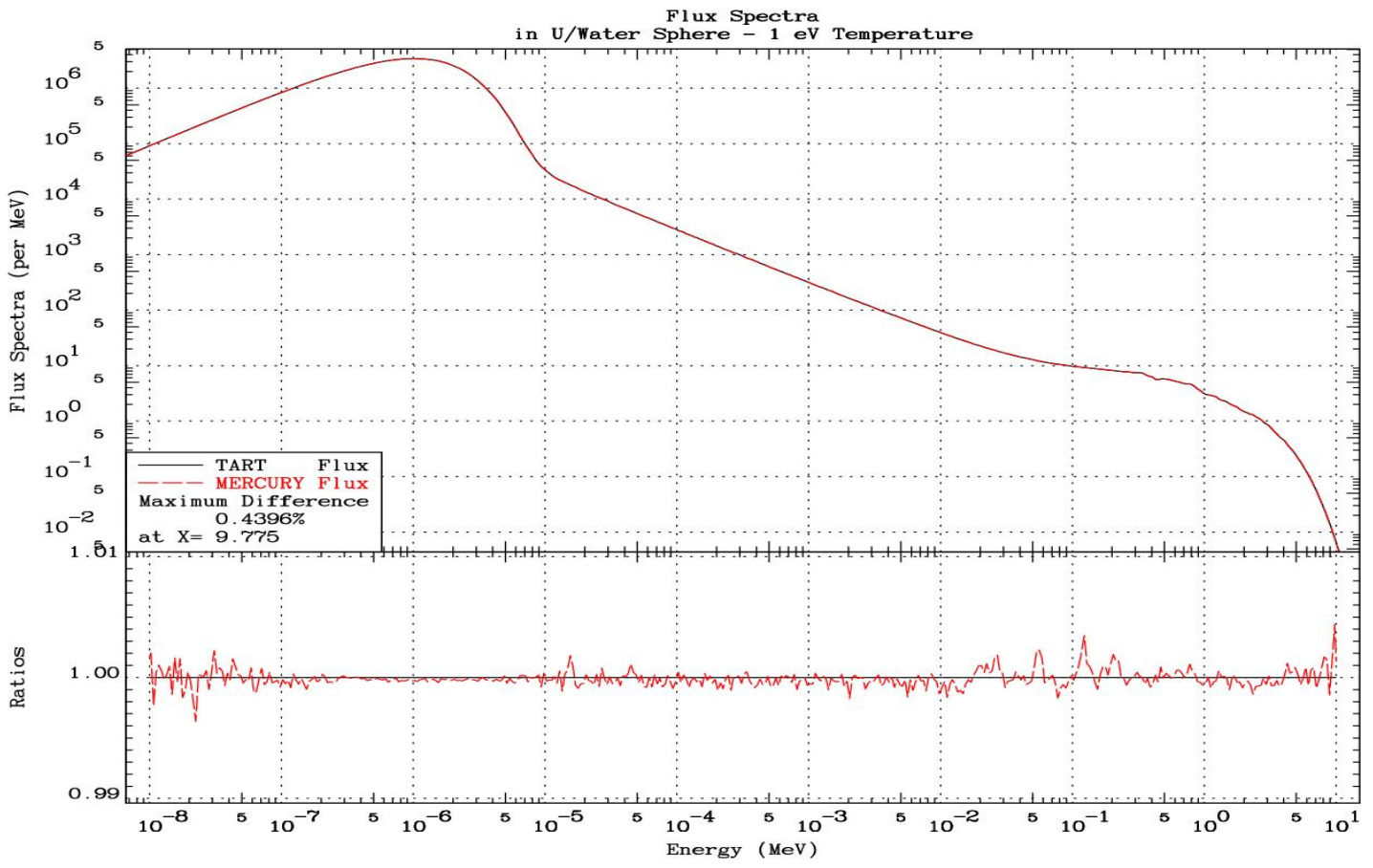

Flux Spectra

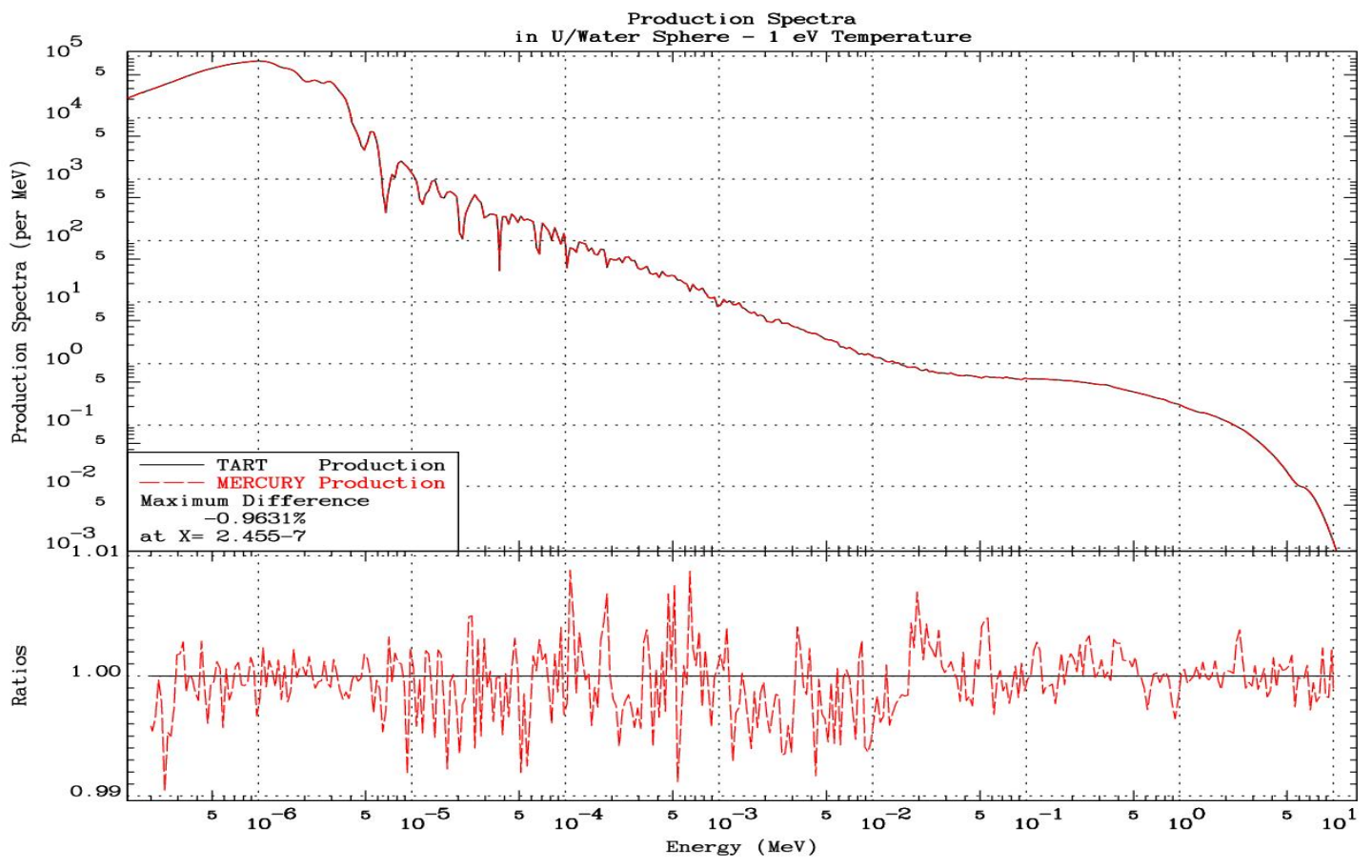

Production Spectra 


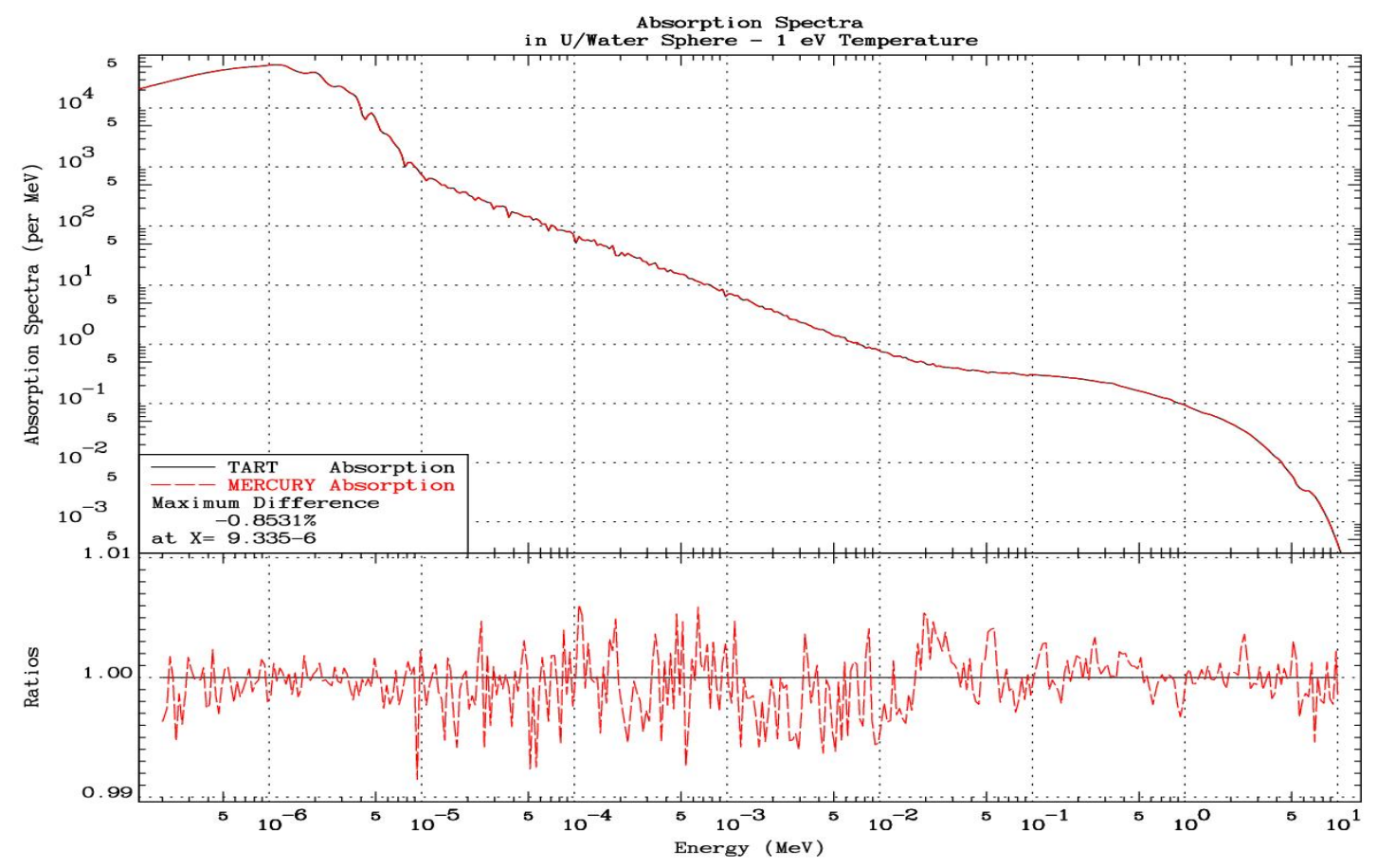

Absorption Spectra

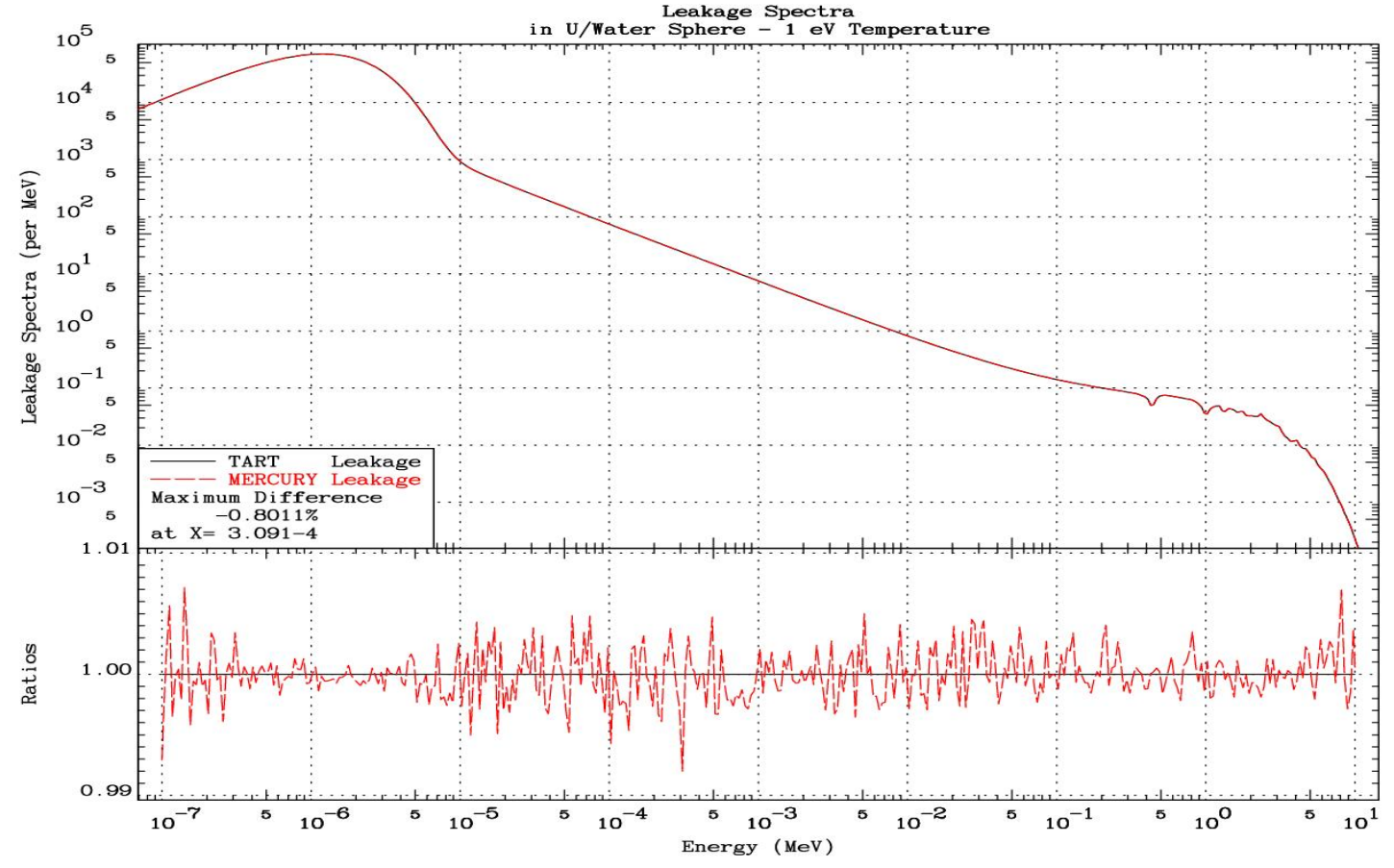

Leakage Spectra 


\section{MERCURY vs. TART: 10 eV Temperature}

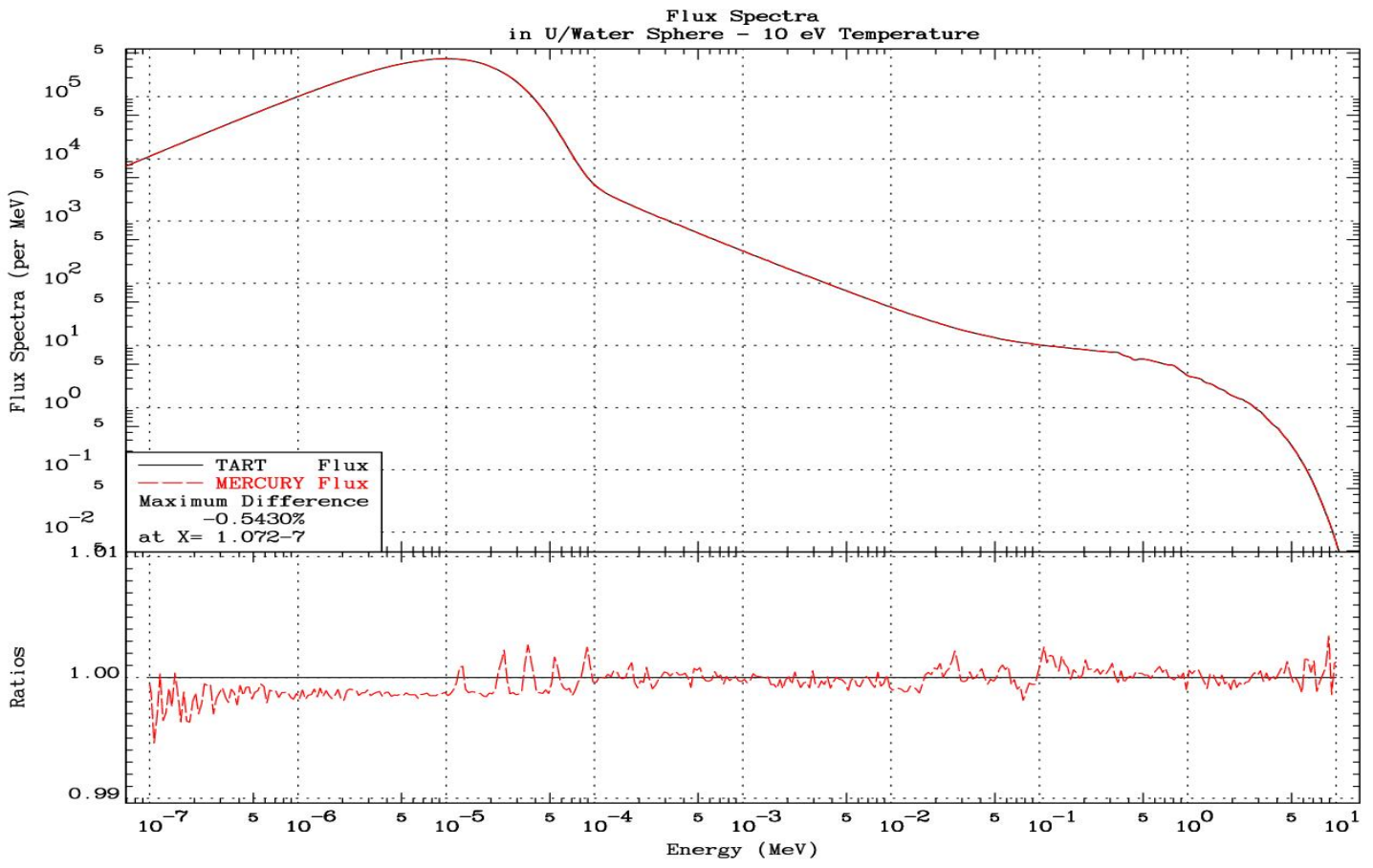

Flux Spectra

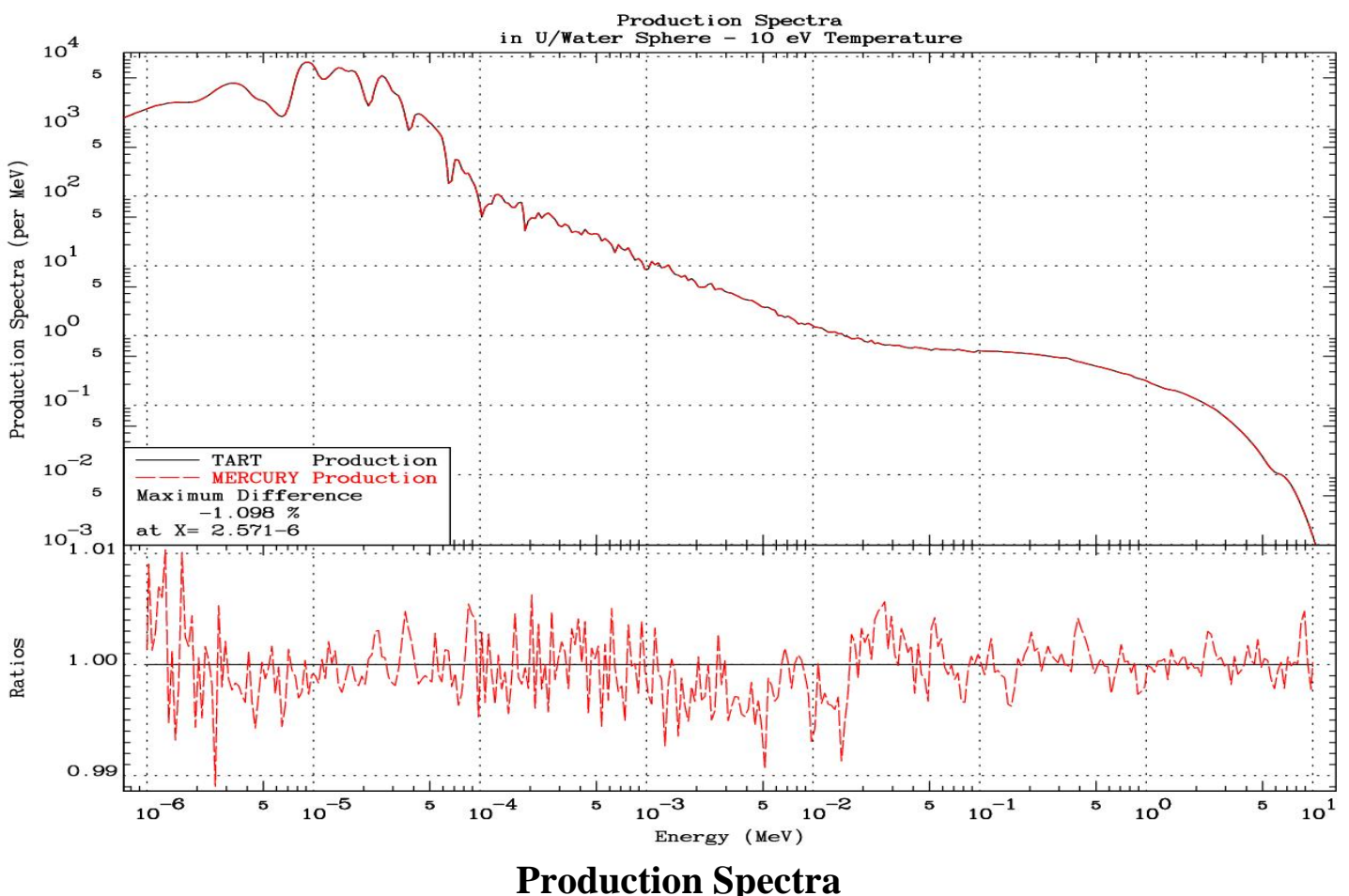




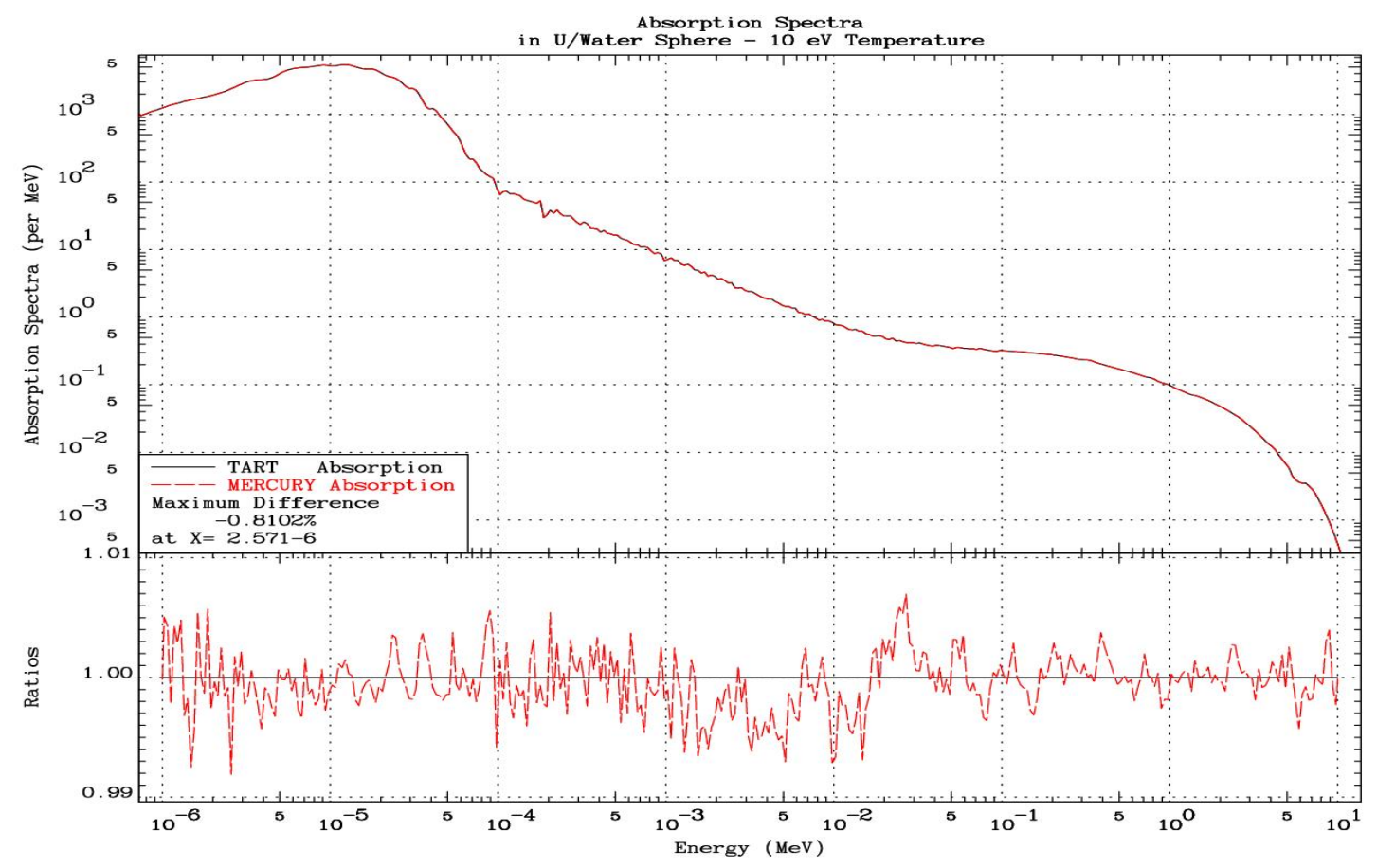

Absorption Spectra

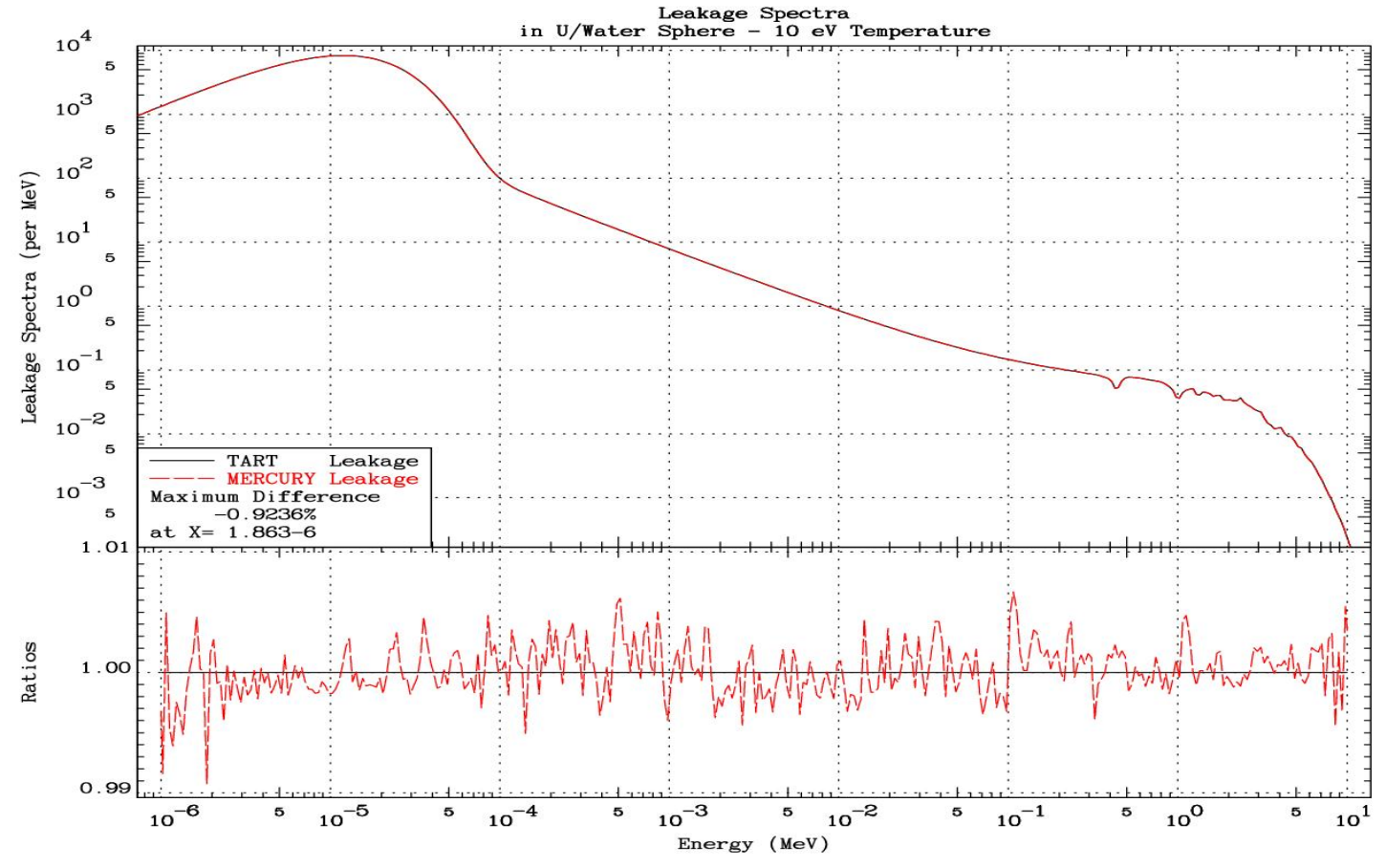

Leakage Spectra 


\section{MERCURY vs. TART: $100 \mathrm{eV}$ Temperature}

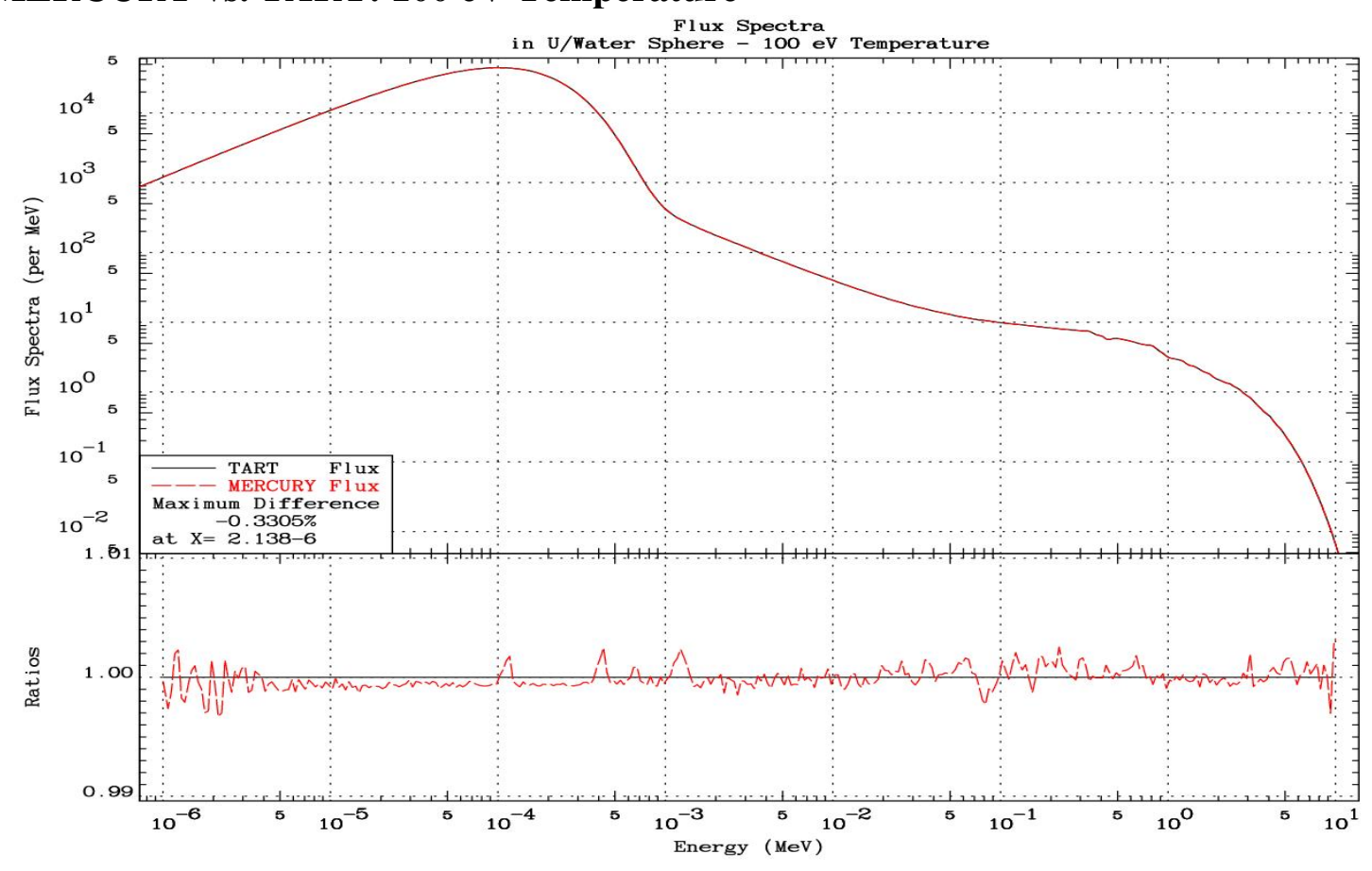

Flux Spectra

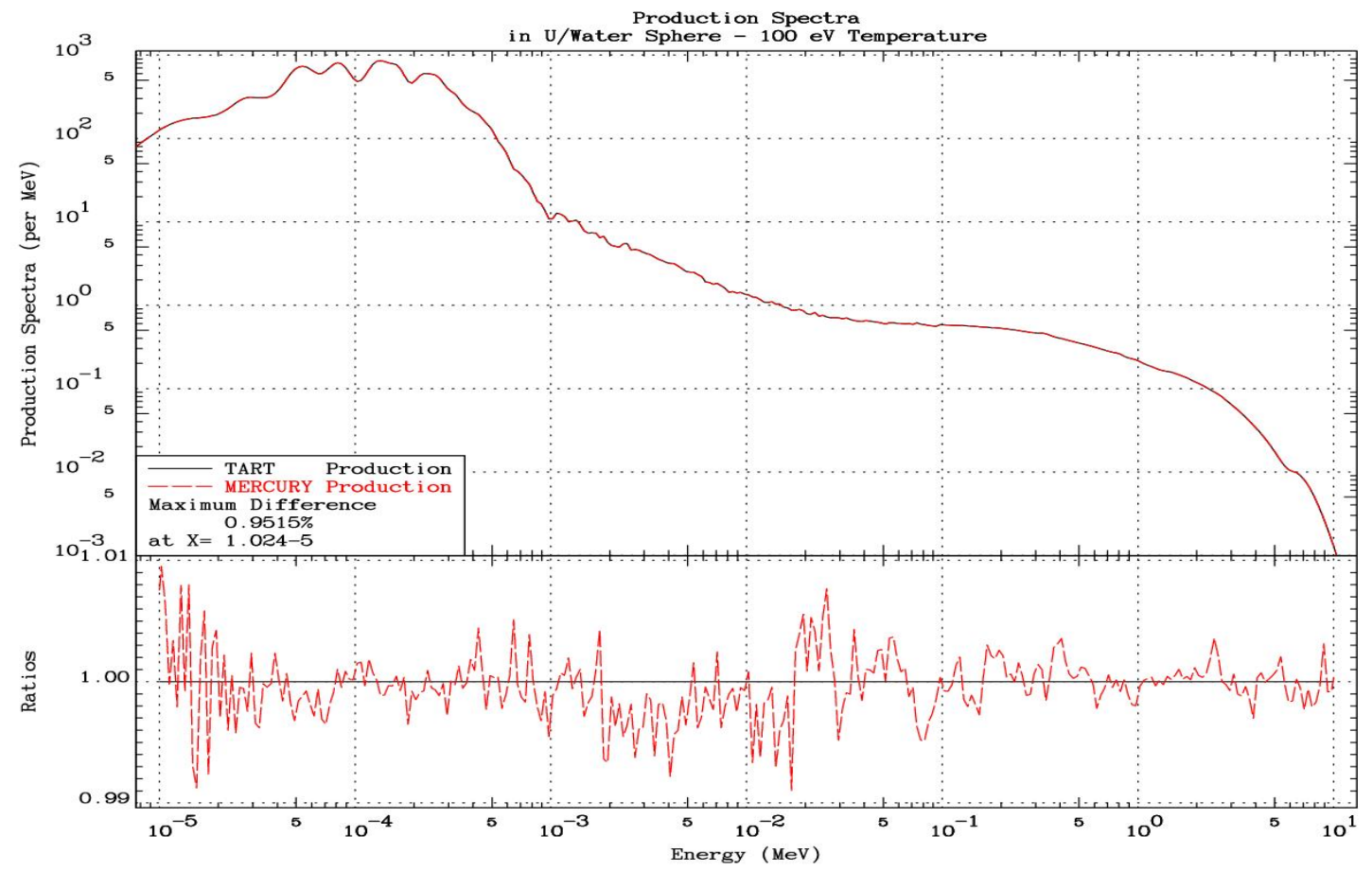

Production Spectra 


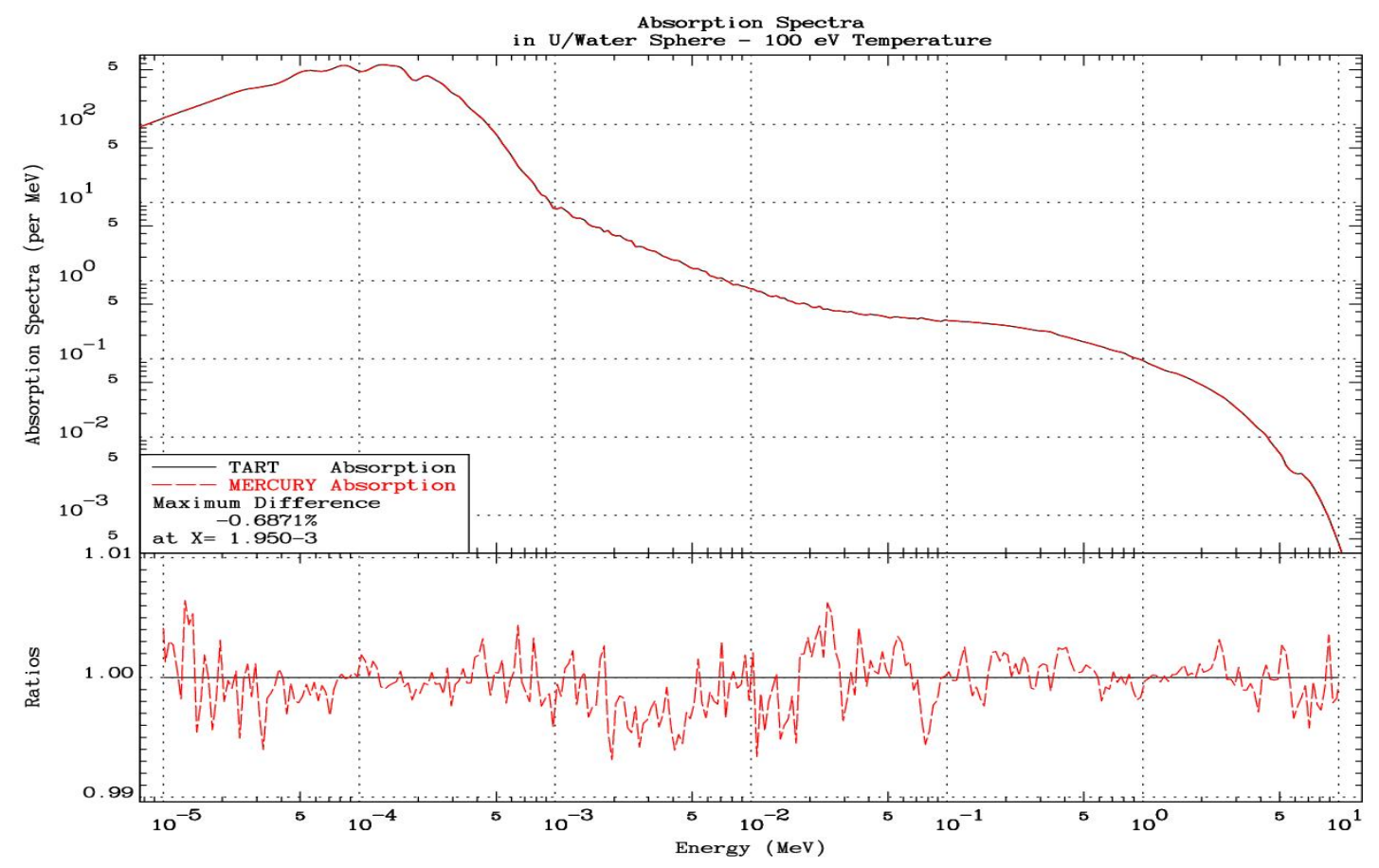

Absorption Spectra

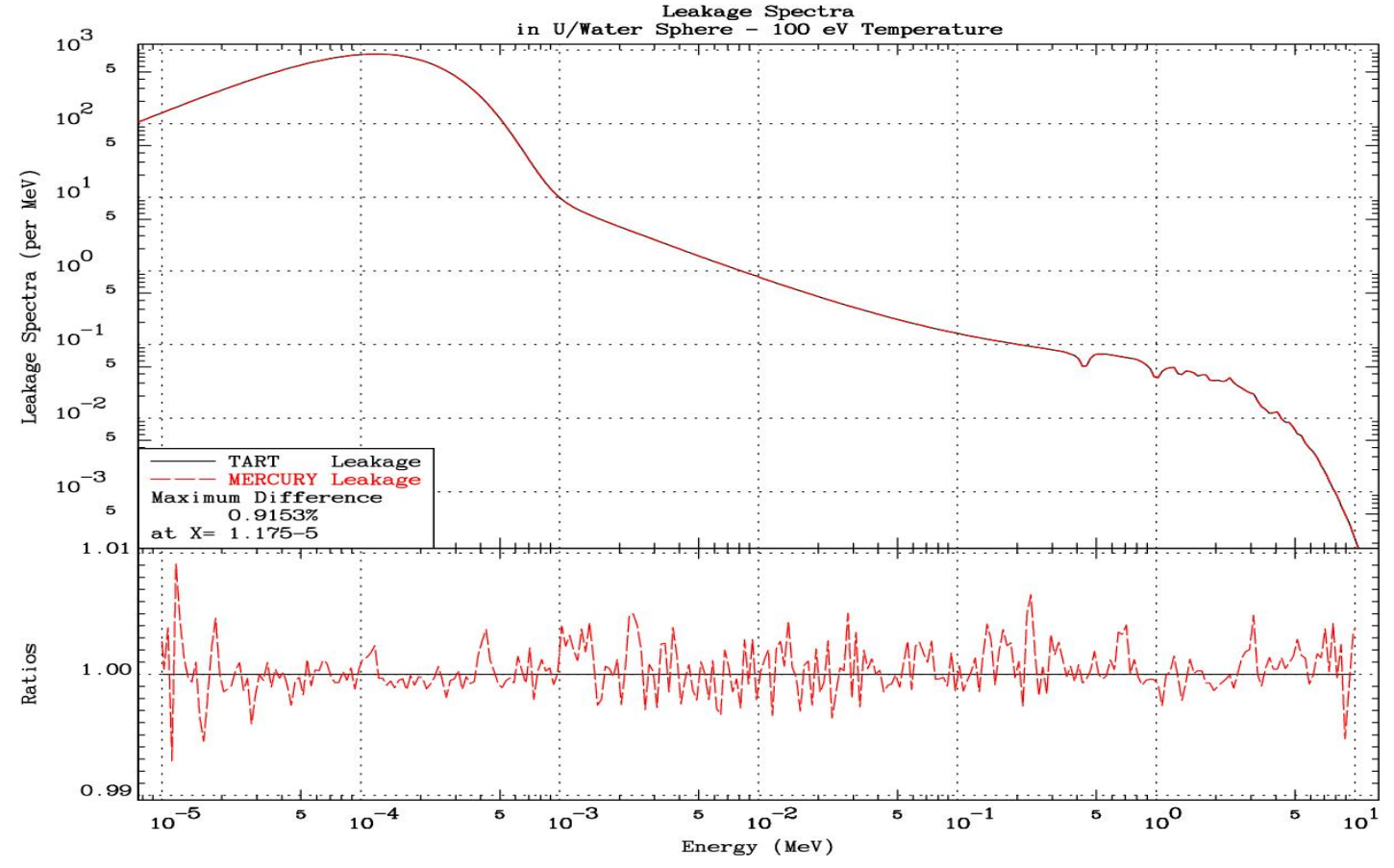

Leakage Spectra 


\section{MERCURY vs. TART: 1000 eV Temperature}

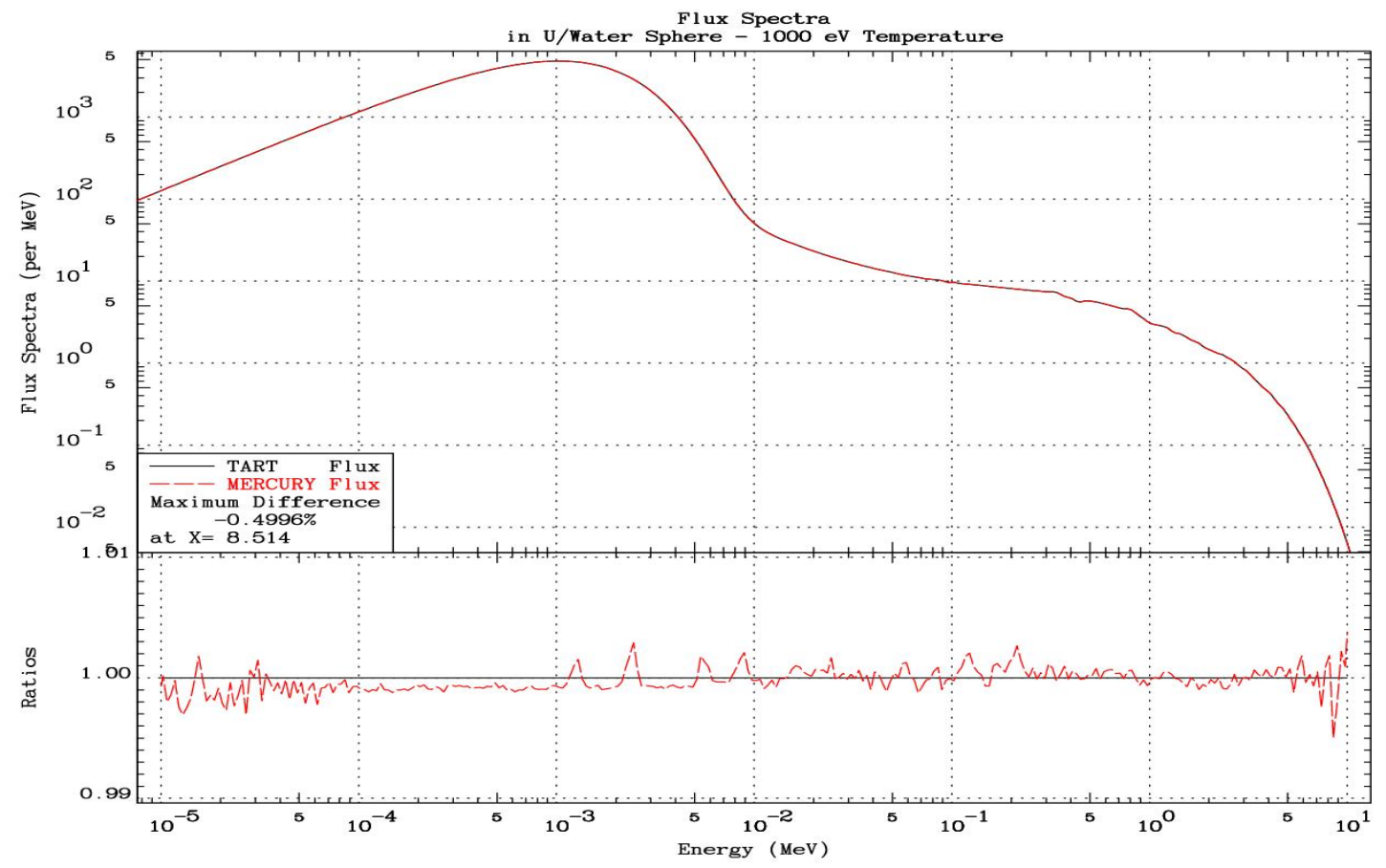

Flux Spectra

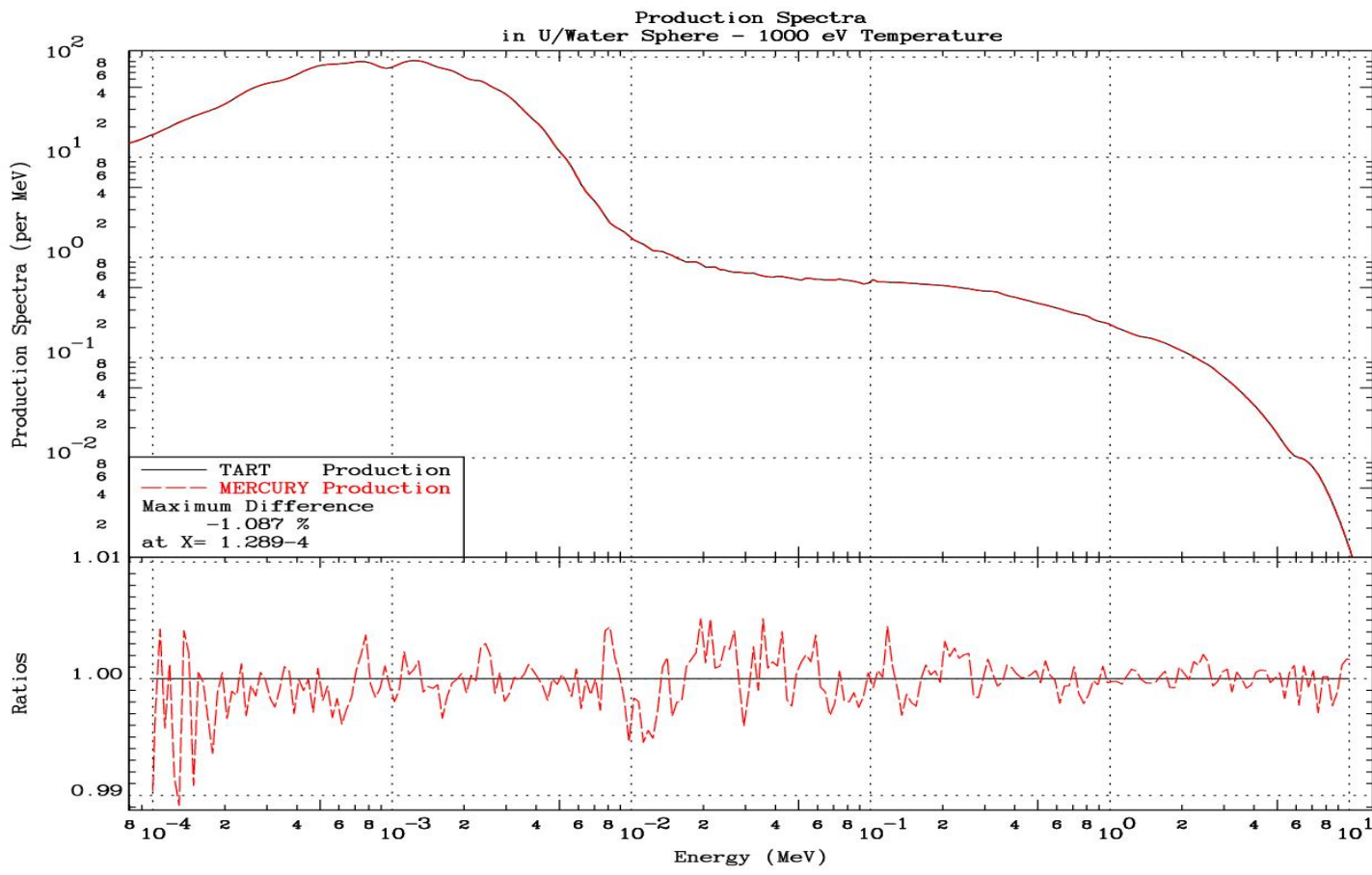

Production Spectra 


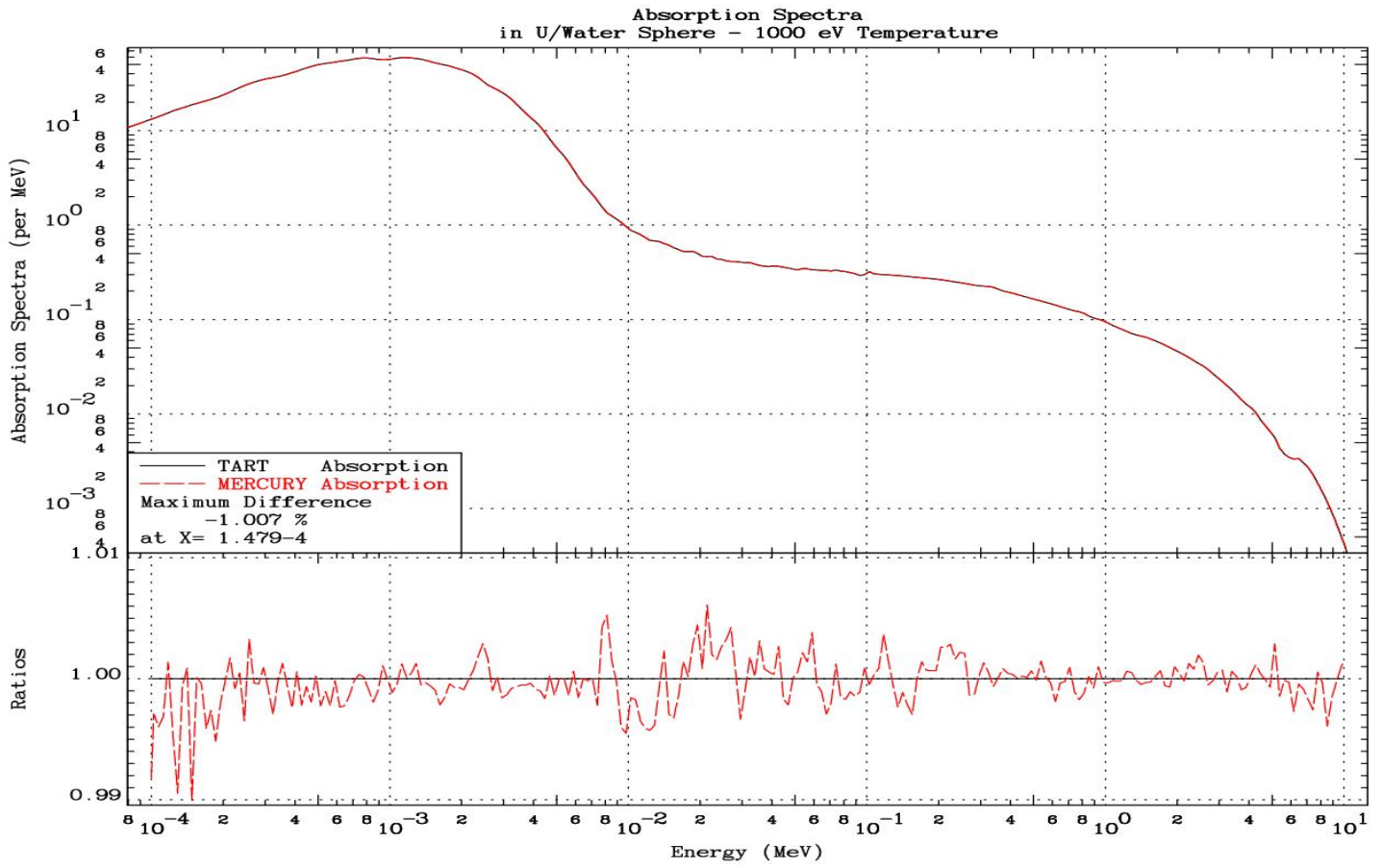

Absorption Spectra

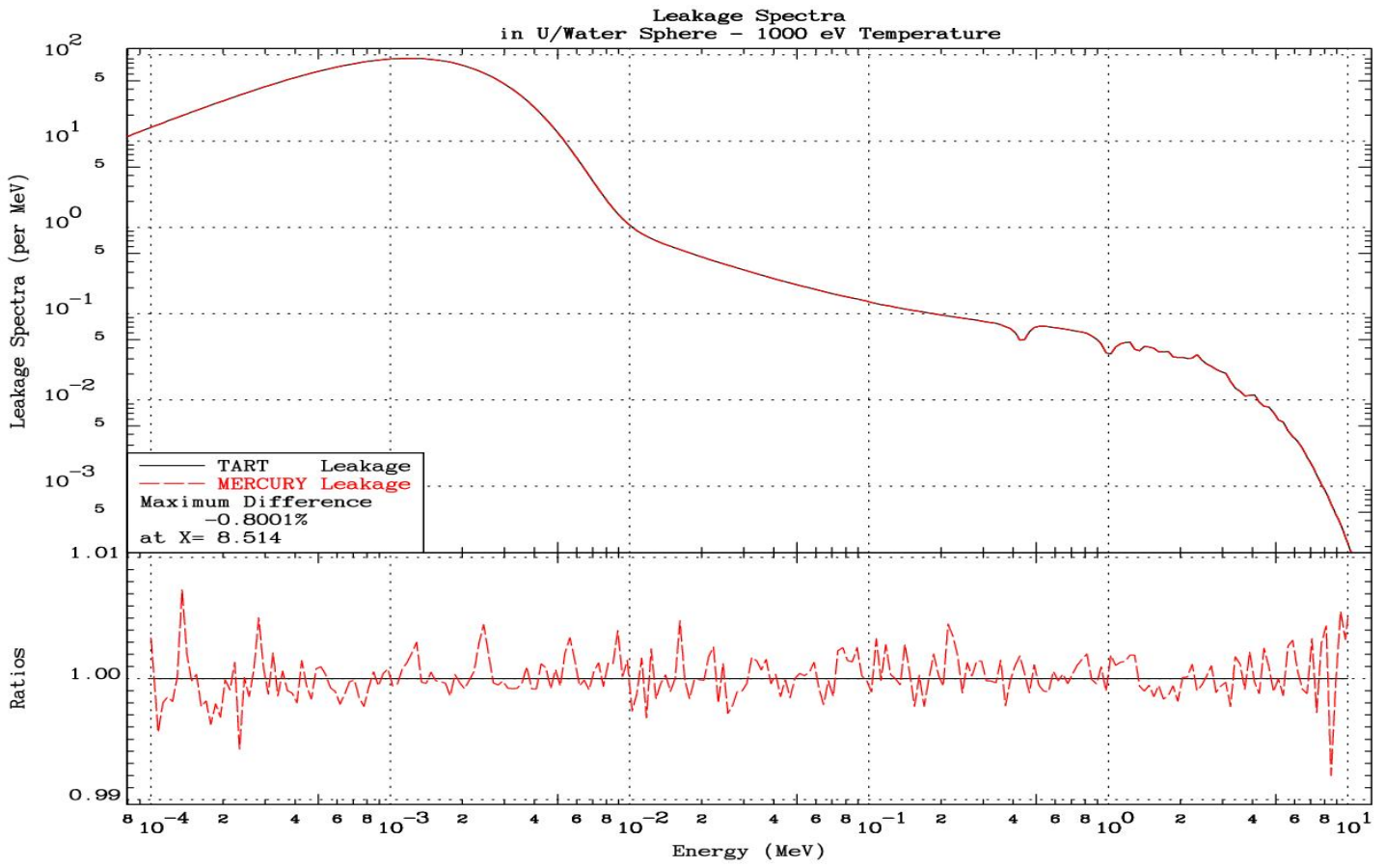

Leakage 


\section{Comparison of Methods: Integral Results}

The above results verify excellent agreement between MERCURY and TART both using exactly the same continuous energy neutron cross sections and methods. But it is important for the reader to understand that this agreement depends crucially on using continuous energy cross sections. In particular do not expect a deterministic code (Sn) [3] using multi-group cross sections to produce the same results as shown above for MERCURY and TART using continuous energy cross sections.

In order to prove this point below we present TART results using several different cross section models: 1) the multi-band method [8,9], that includes the effect of spatial and directional self-shielding, 2) the multi-group method [9], that as used here does not include the effect of self-shielding; see appendix B for details of self-shielding. In all of these cases the calculations used the TART 616 group structure.

We can summarize the integral results by stating that the multi-band results are in just as good agreement with the TART continuous energy results as are the MERCURY continuous energy results, i.e., the multi-band method is accurately re-producing the important self-shielding effects. In contrast the multi-group results show differences more than an order of magnitude bigger. Note in particular that for the room temperature case $(0.0253 \mathrm{eV})$ the multi-group results predict that this system is slightly sub-critical, whereas all of the other results predict it is super-critical; here the difference in K-eff, is near $0.3 \%$, about three times what we consider to be an acceptable difference.

But it is important to note that with increasing temperature the agreement between continuous energy and multi-group results improve, as the cross sections become smoother and self-shielding becomes less important.

TART Multi-Band Results

Red indicates difference from TART Continuous Energy results

\begin{tabular}{|c|c|c|c|c|c|}
\hline $\begin{array}{c}\text { Temperature } \\
(\mathbf{e V})\end{array}$ & Absorption & Leakage & K-eff & $\begin{array}{c}\text { Removal Time } \\
(\mu \text { sec })\end{array}$ & $\begin{array}{c}\text { Alpha } \\
(1 / \text { usec })\end{array}$ \\
\hline $0.0253 \mathrm{eV}$ & $\mathbf{0 . 5 8 2 6 7}$ & $\mathbf{0 . 4 1 4 6 3}$ & 1.00271 & 23.757 & $1.1409 \mathrm{~d}-4$ \\
\hline $1 \mathrm{eV}$ & $\mathbf{0 . 5 1 7 0 0}$ & $\mathbf{0 . 4 5 8 2 7}$ & 1.02535 & $\mathbf{6 . 3 7 5 0}$ & $\mathbf{3 . 9 7 7 2 d - 3}$ \\
\hline $10 \mathrm{eV}$ & $\mathbf{0 . 5 3 1 2 2}$ & $\mathbf{0 . 4 8 6 5 0}$ & $\mathbf{0 . 9 8 2 5 8}$ & 2.2980 & $-7.5790 \mathrm{~d}-3$ \\
\hline $100 \mathrm{eV}$ & $\mathbf{0 . 5 0 8 8 6}$ & $\mathbf{0 . 4 7 6 1 1}$ & $\mathbf{1 . 0 1 5 2 6}$ & $\mathbf{0 . 8 2 7 0 8}$ & $\mathbf{1 . 8 4 5 5 d - 2}$ \\
\hline $1000 \mathrm{eV}$ & $\mathbf{0 . 4 9 3 6 4}$ & $\mathbf{0 . 4 6 1 4 4}$ & $\mathbf{1 . 0 4 7 0 4}$ & $\mathbf{0 . 2 9 9 3 2}$ & $\mathbf{1 . 5 7 1 5 d - 1}$ \\
\hline
\end{tabular}

TART Multi-Group Results

Red indicates difference from TART Continuous Energy results

\begin{tabular}{|c|c|c|c|c|c|}
\hline $\begin{array}{c}\text { Temperature } \\
(\mathrm{eV})\end{array}$ & Absorption & Leakage & K-eff & $\begin{array}{c}\text { Removal Time } \\
(\mu \mathrm{sec})\end{array}$ & $\begin{array}{l}\text { Alpha } \\
\text { (1/usec) }\end{array}$ \\
\hline $0.0253 \mathrm{eV}$ & 0.58477 & 0.41580 & 0.99944 & 23.734 & $-2.3686 d-5$ \\
\hline $1 \mathrm{eV}$ & 0.51764 & 0.45862 & 1.02438 & 6.3788 & $3.8222 \mathrm{~d}-3$ \\
\hline $10 \mathrm{eV}$ & 0.53149 & 0.48646 & 0.98237 & 2.2993 & $-7.6662 d-3$ \\
\hline $100 \mathrm{eV}$ & 0.50889 & 0.47581 & 1.01553 & 0.82735 & $1.8773 d-2$ \\
\hline $1000 \mathrm{eV}$ & 0.49365 & 0.46127 & 1.04720 & 0.29945 & $1.5763 d-1$ \\
\hline
\end{tabular}




\section{Comparison of Methods: Differential Results}

As stated above, these days it is not sufficient to only reproduce integral parameters such as K-eff, we should also compare differential results. When we do this we can better understand the difficulty that the multi-group method has reproducing continuous energy results. The plots below show a comparison of the spectra for the three methods. Note the problems that the multi-group method has in the resolved resonance region of uranium. In our continuous energy MERCURY to TART comparisons we found excellent agreement between differential results, with little more than noise fluctuating about the $+/-0.1 \%$ level. Here we see substantial differences that differ over considerable energy ranges. We now see LARGE differences, particularly for the absorption spectra: for the room temperature $(0.0253 \mathrm{eV})$ case we see absorption ratios that are up to $20 \%$ too high and $30 \%$ too low. We can see from the above integral results that these effects do not cancel out, and the overall effect is for the multi-group method to over-predict the absorption, leading to the prediction that this system is sub-critical.

As temperature increases and the cross sections become smoother and the agreement improves. By $100 \mathrm{eV}$ temperature the agreement through the resonance region is very good.

A separate point to note is that at all temperatures we see differences at high energy, particularly in the leakage. This indicates that the 50 groups per decade that we are using here is not adequate to accurately represent the high energy oxygen cross sections in the water. The so called oxygen resonances shown in the below figure are at such high energies that temperature has little effect on them, so that this problem is seen in our results at all temperatures.

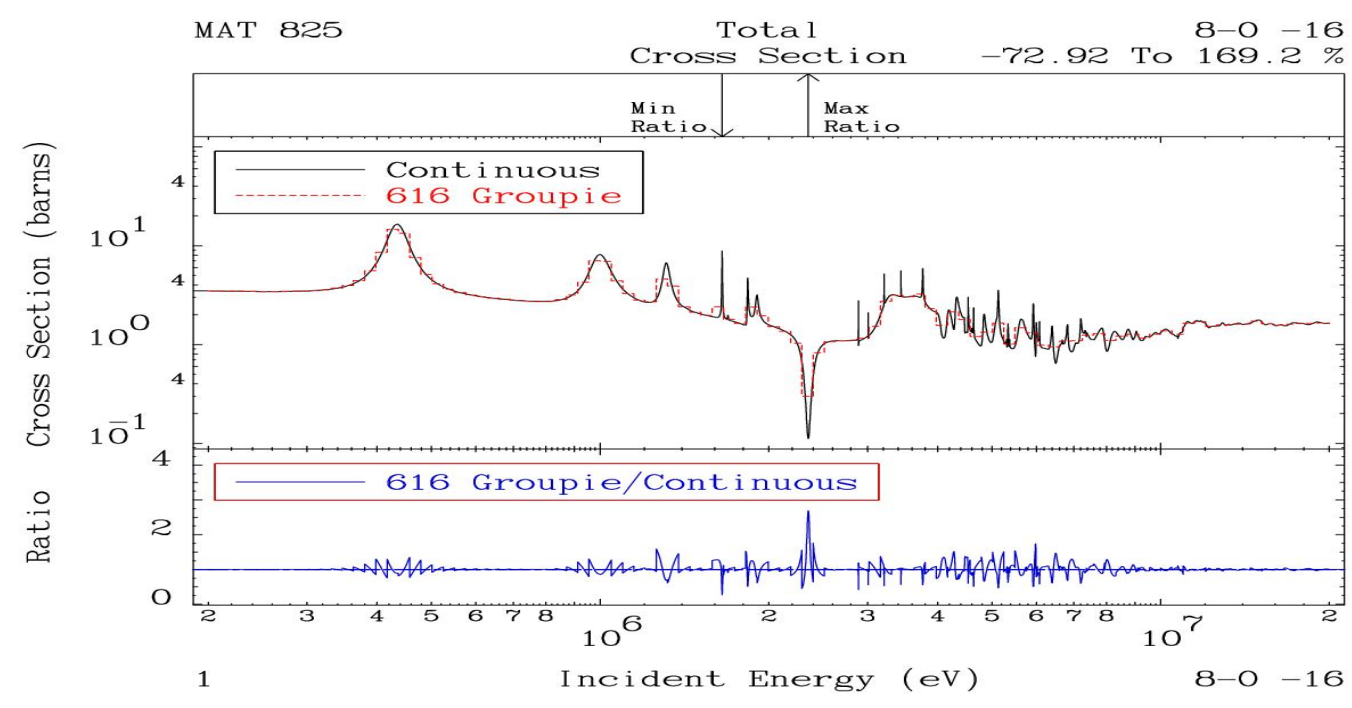

Below are the TART differential results using three different methods. By comparing these results to the above TART vs. MERCURY comparisons you can see the difficulty that the multi-group method is having. 


\section{Comparison of Methods : Thermal $(0.0253 \mathrm{eV})$ Temperature}

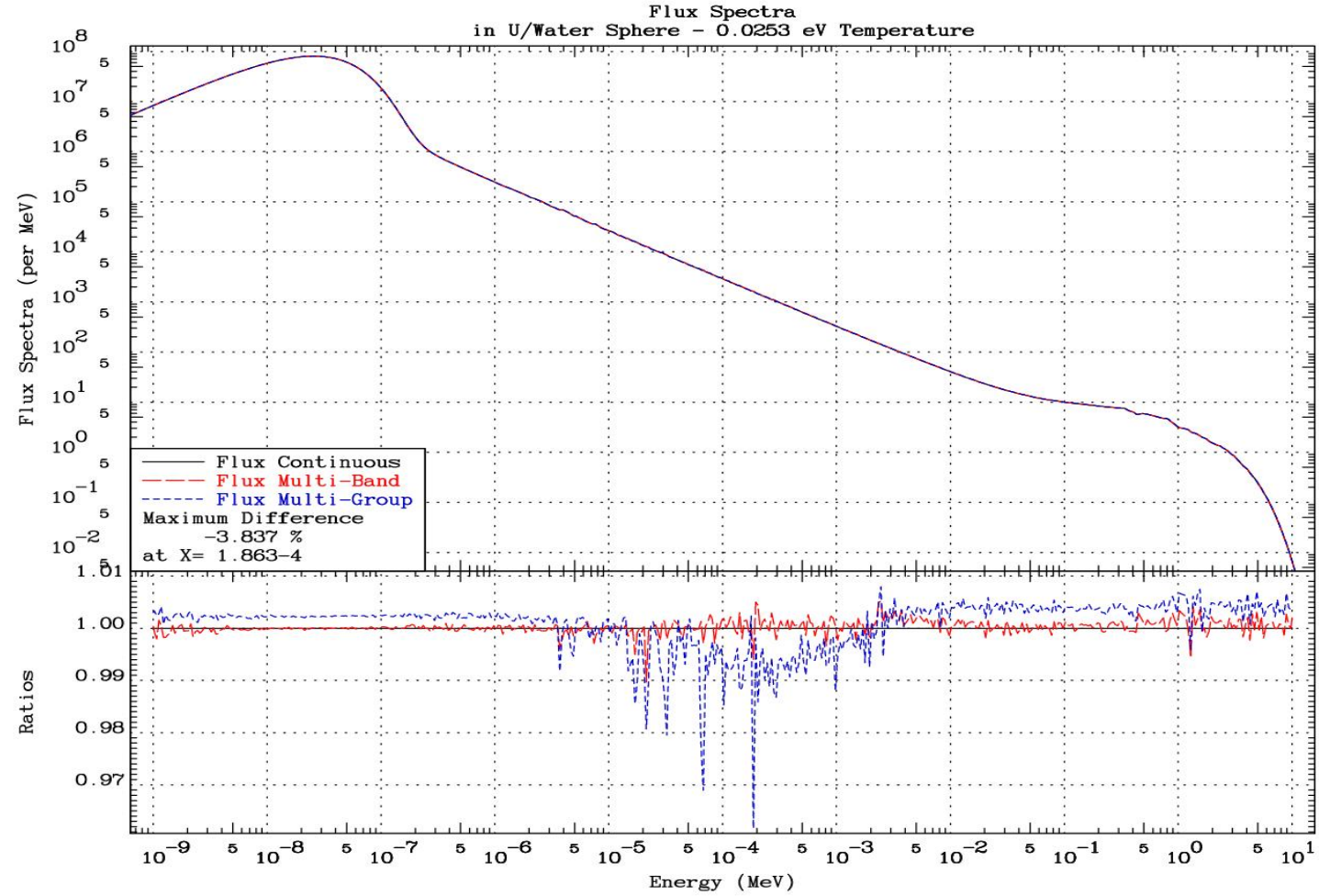

Flux Spectra

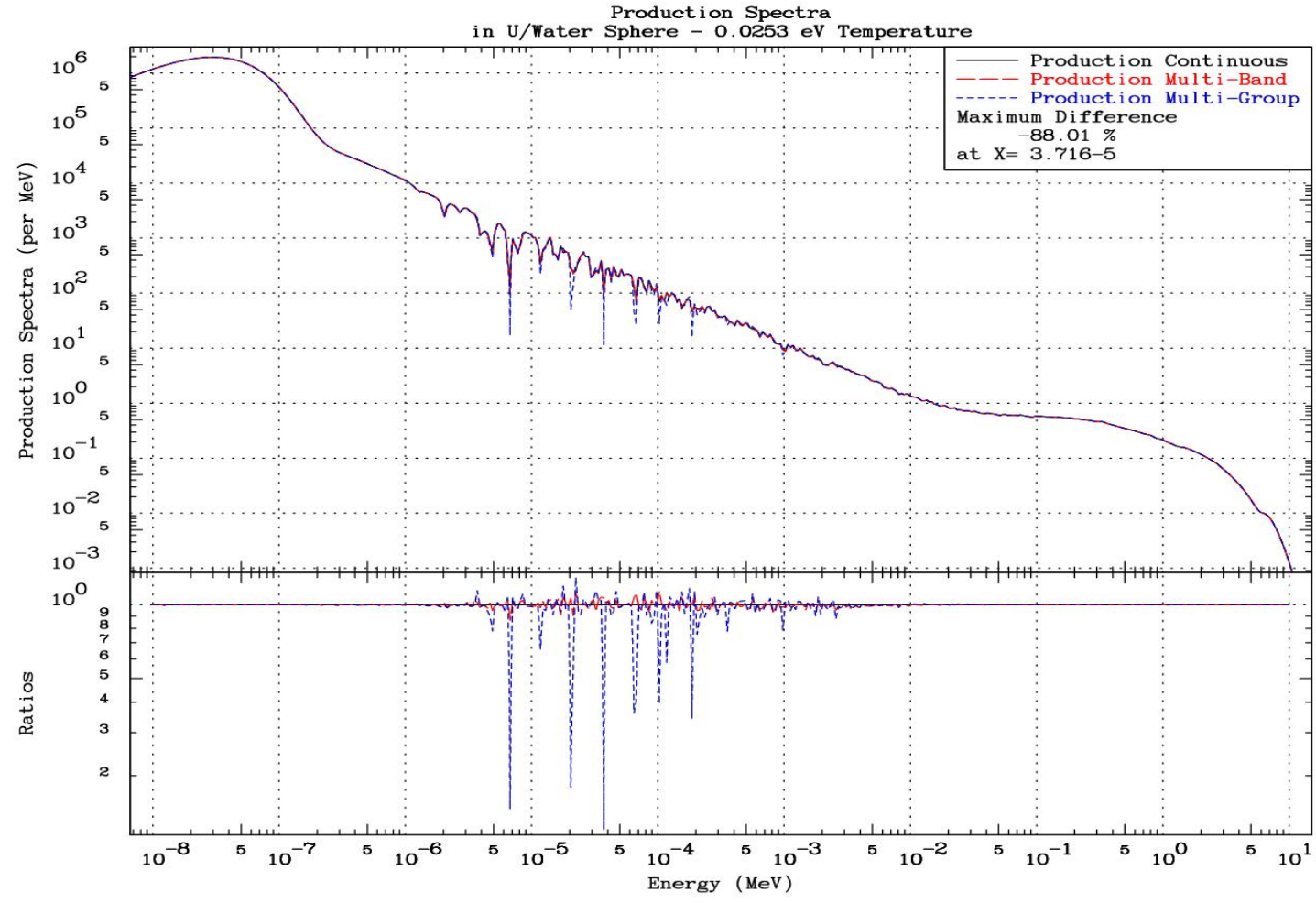

Production Spectra 


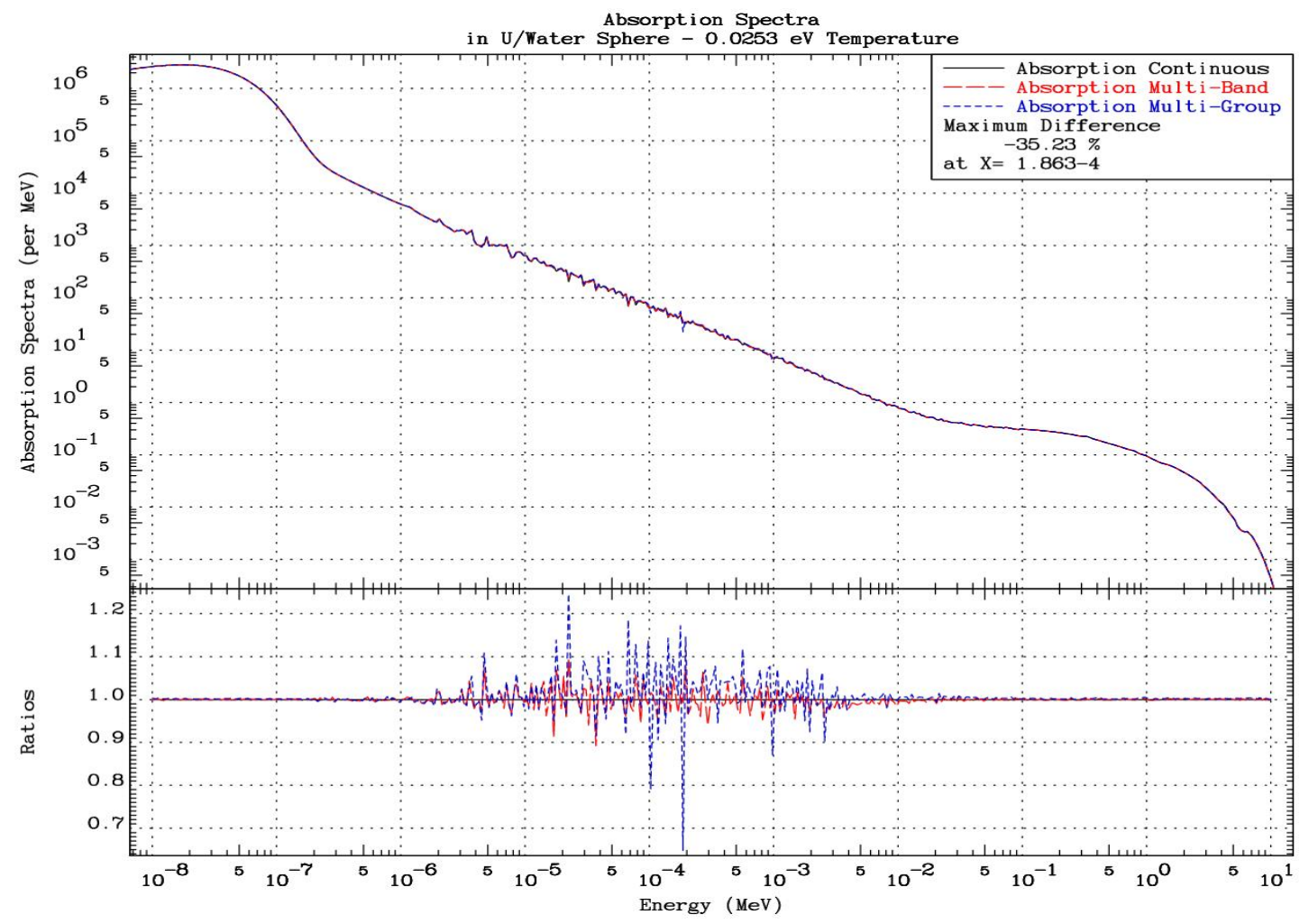

Absorption Spectra

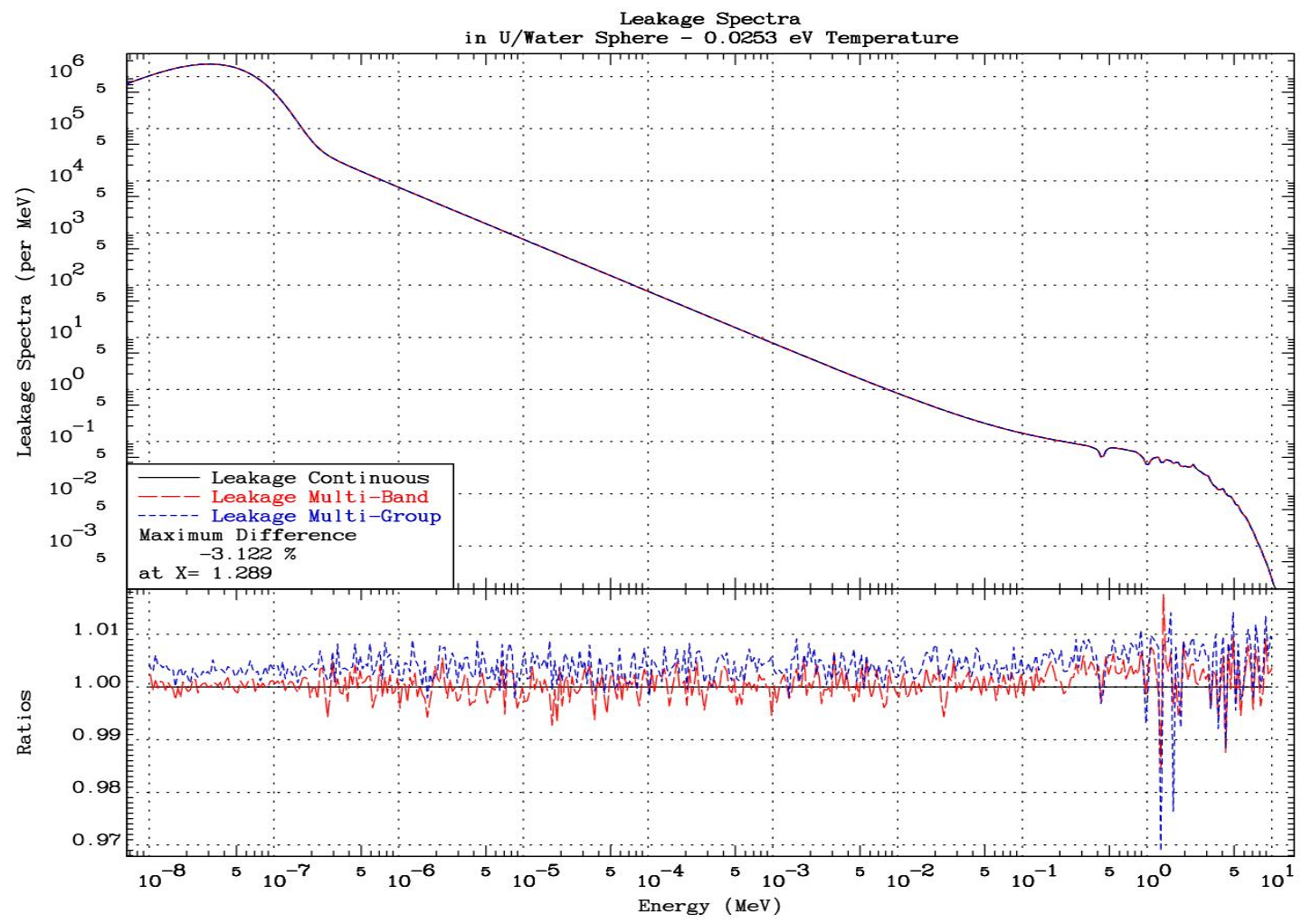

\section{Leakage Spectra}




\section{Comparison of Methods : $1 \mathrm{eV}$ Temperature}

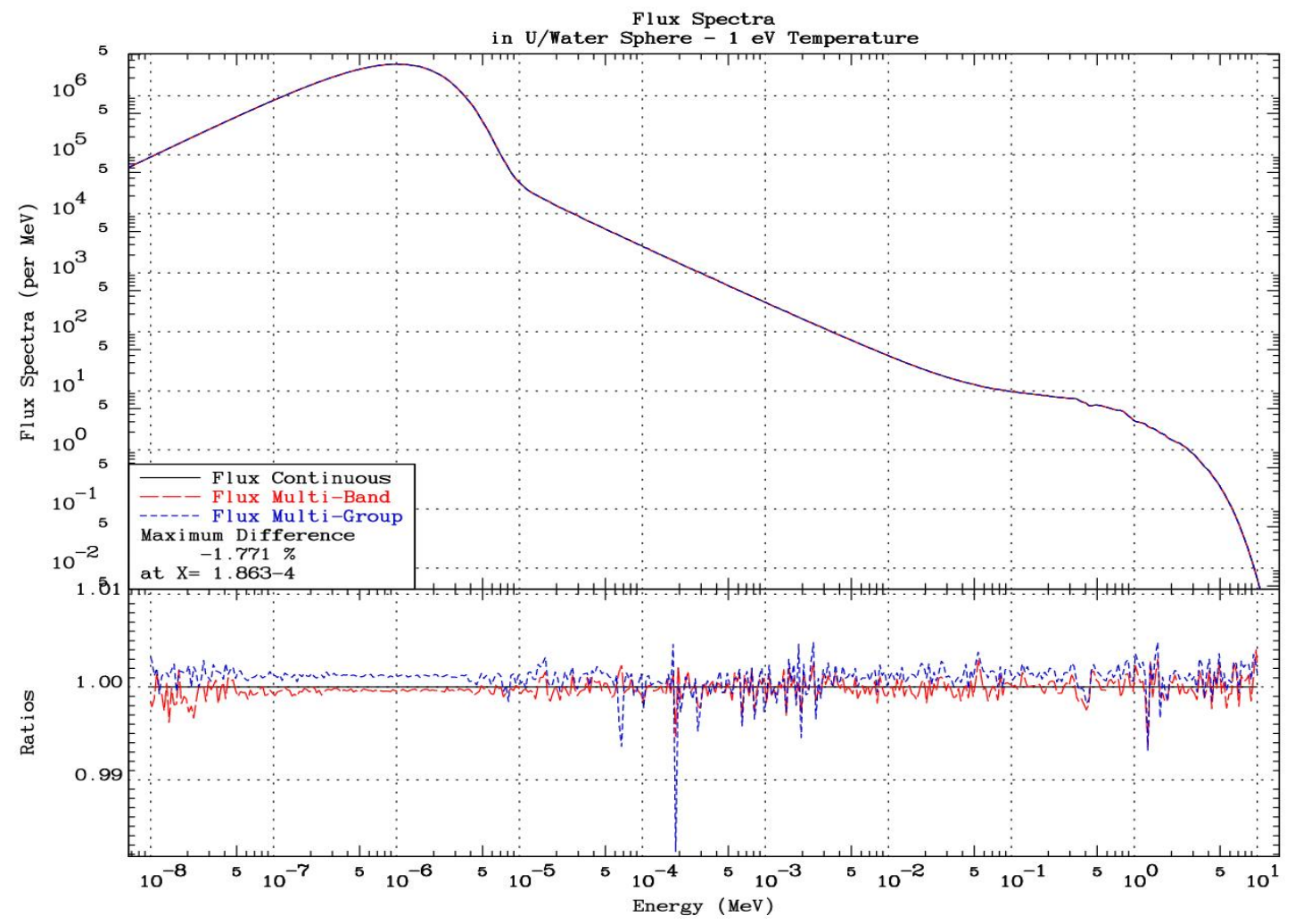

Flux Spectra

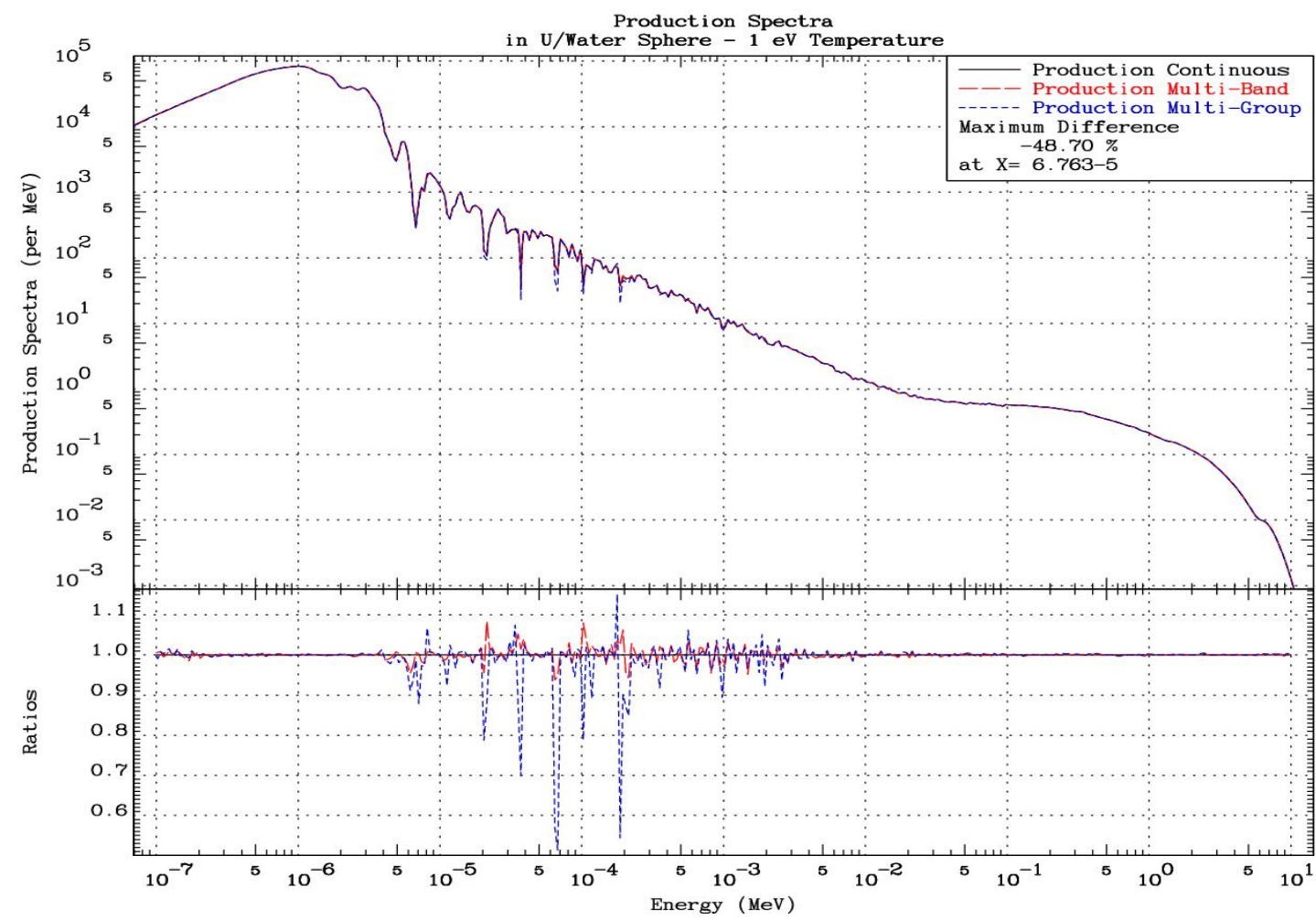

Production Spectra 


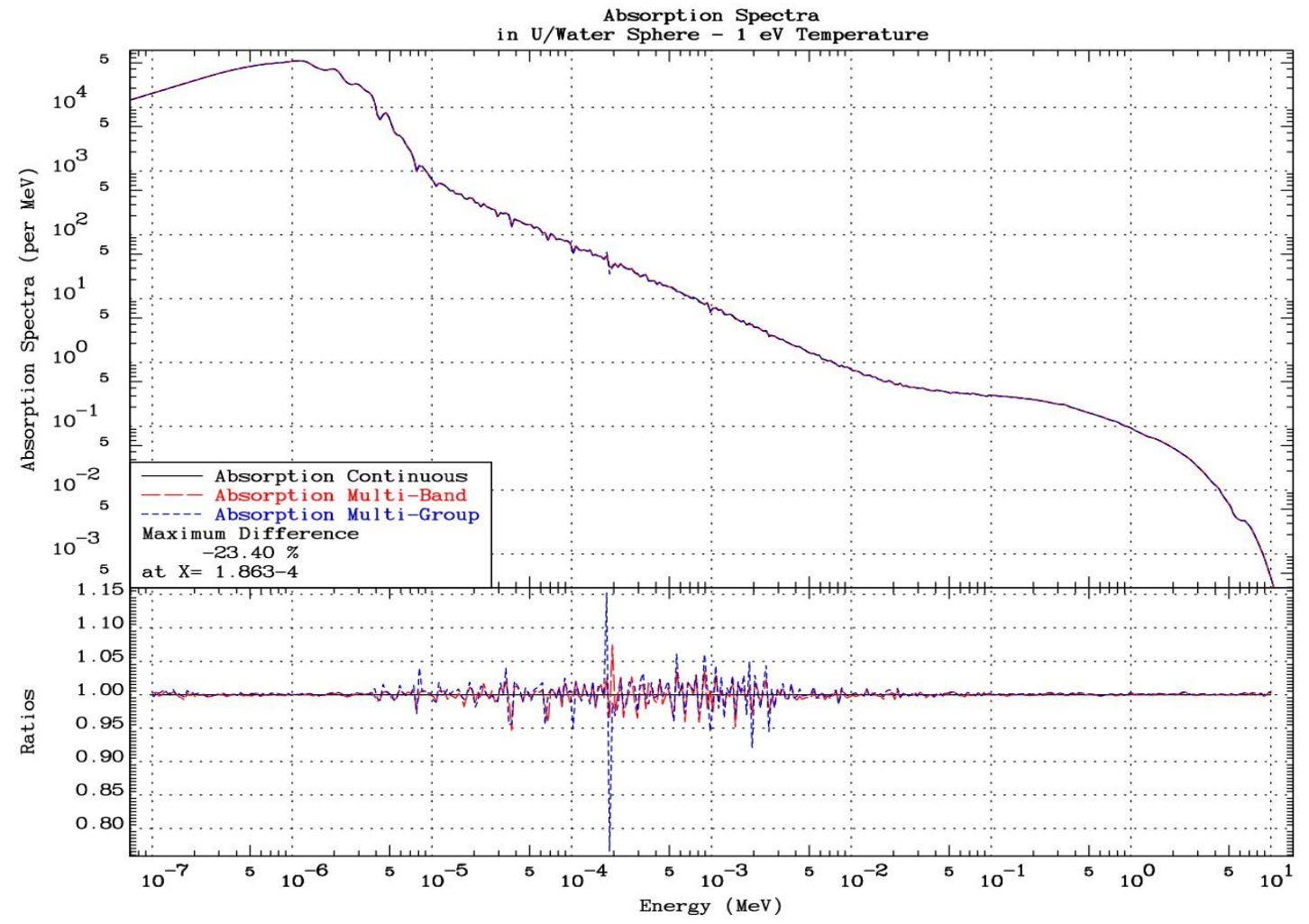

Absorption Spectra

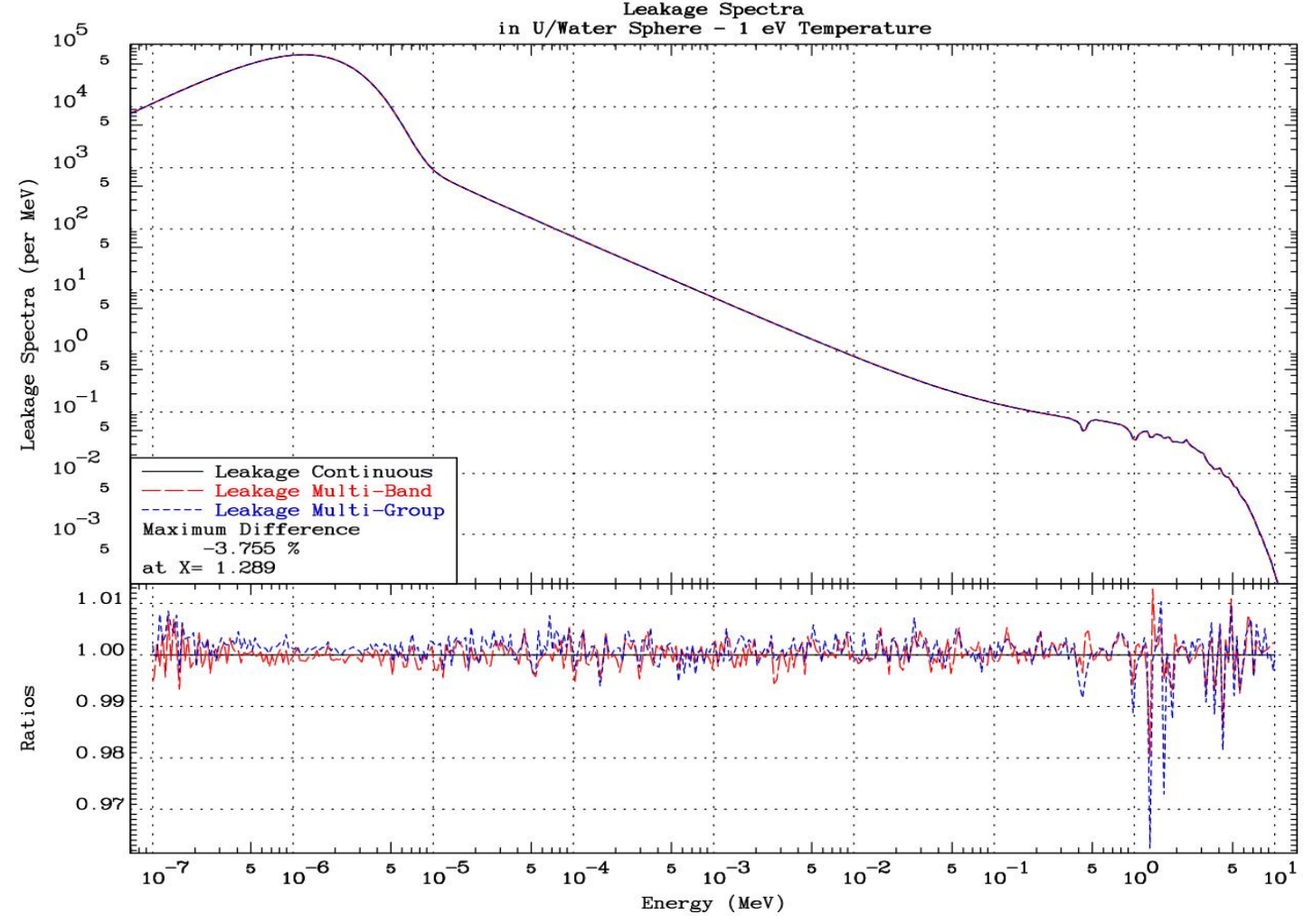

Leakage Spectra 


\section{Comparison of Methods : $10 \mathrm{eV}$ Temperature}

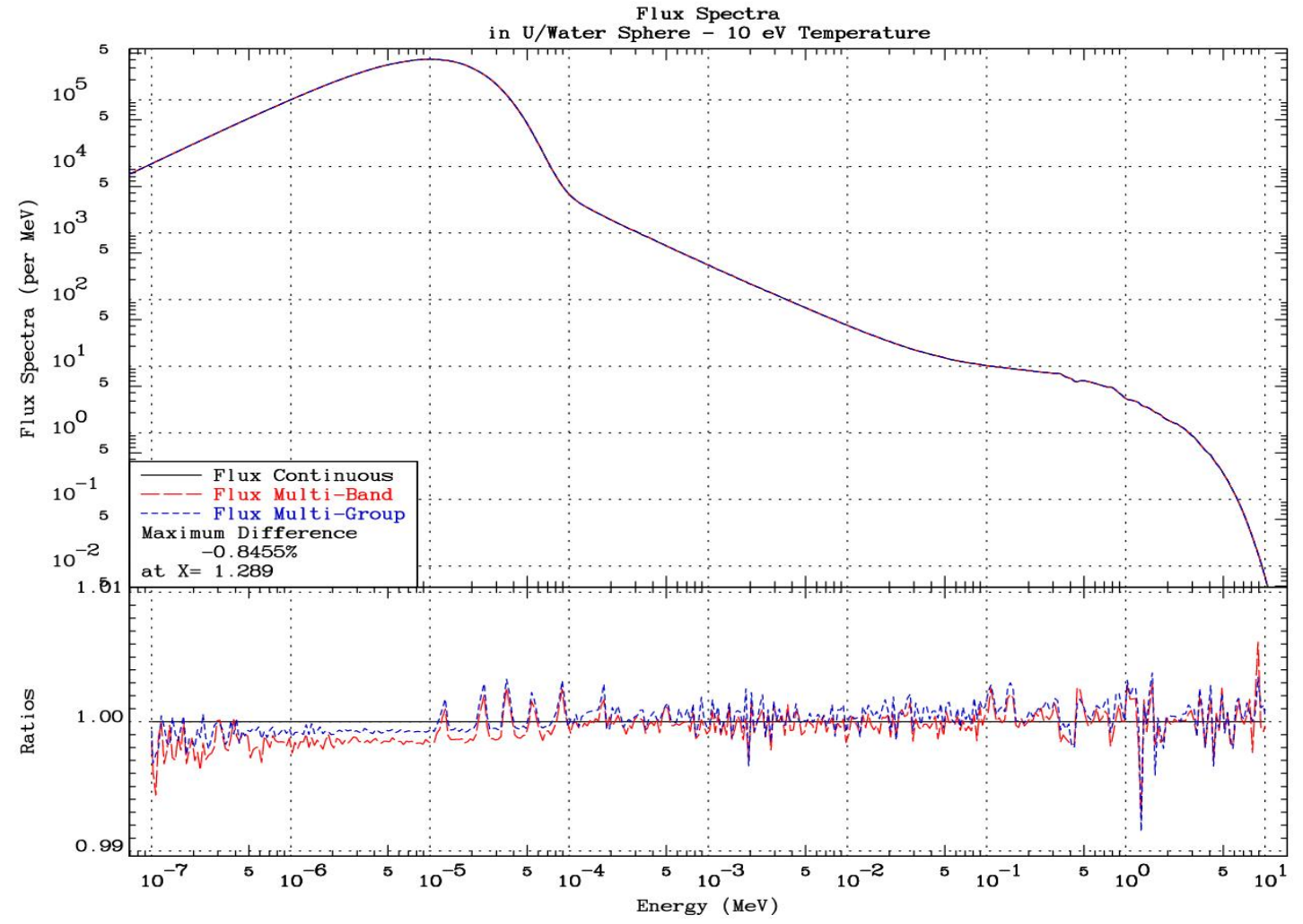

Flux Spectra

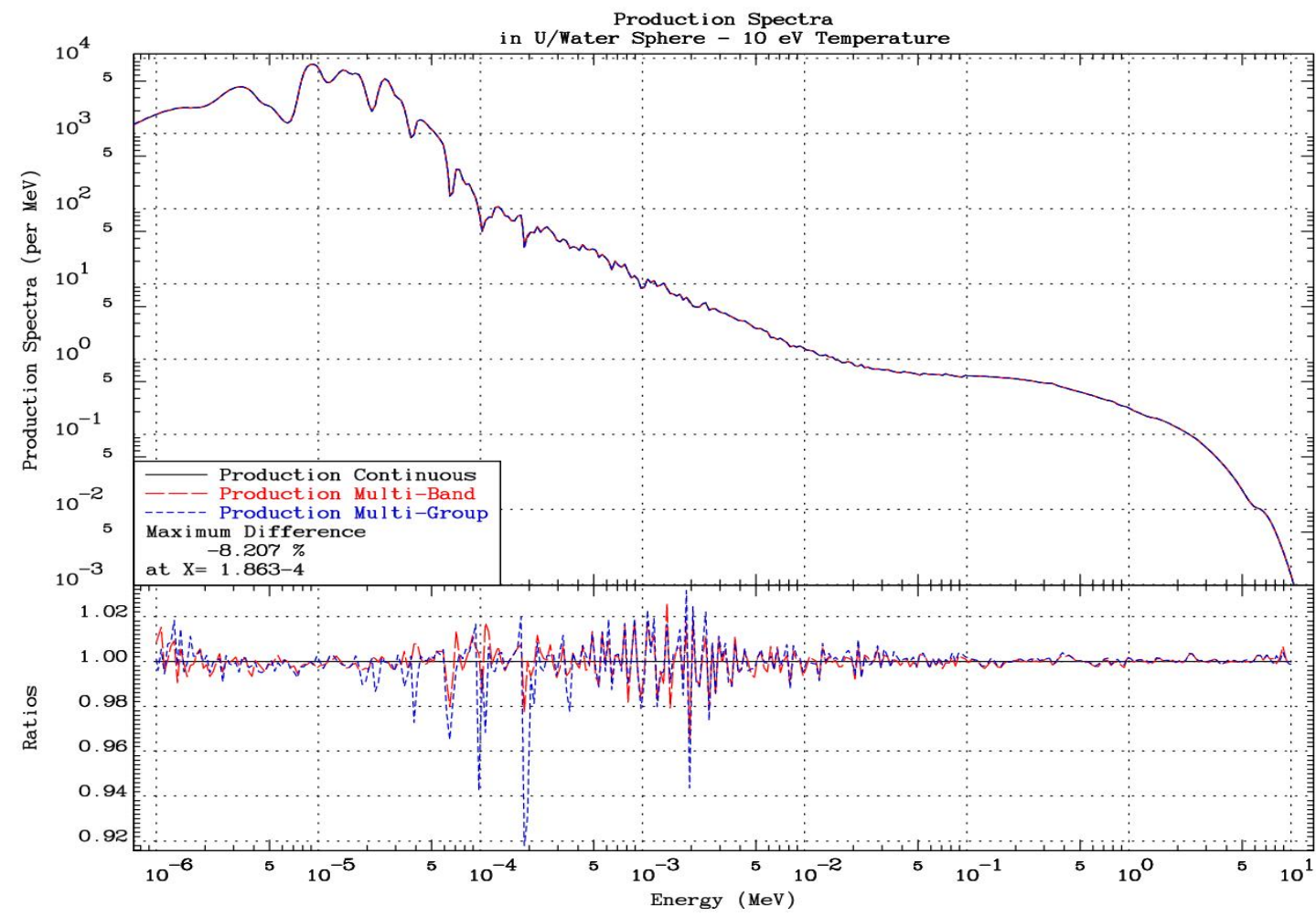

Production Spectra 


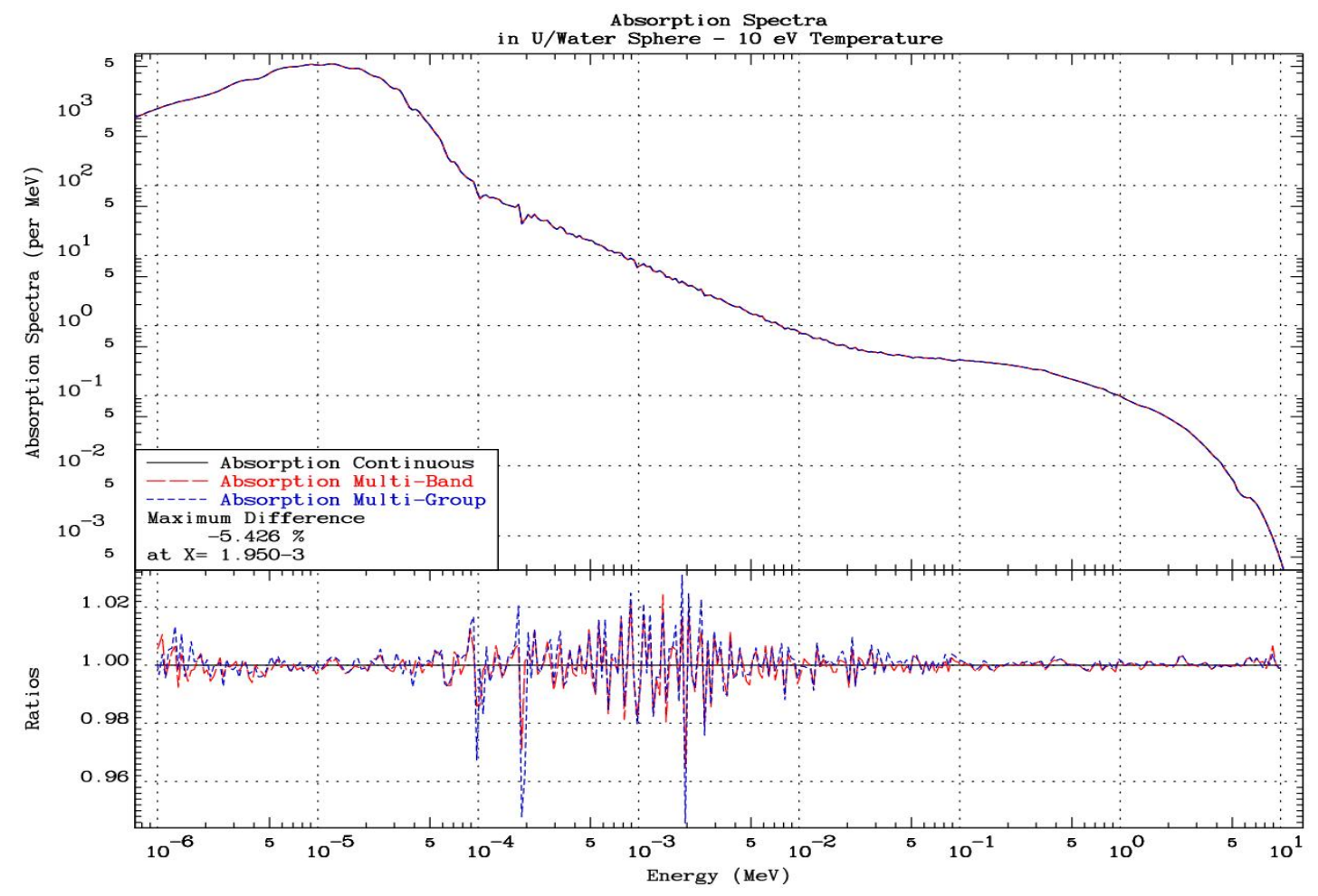

Absorption Spectra

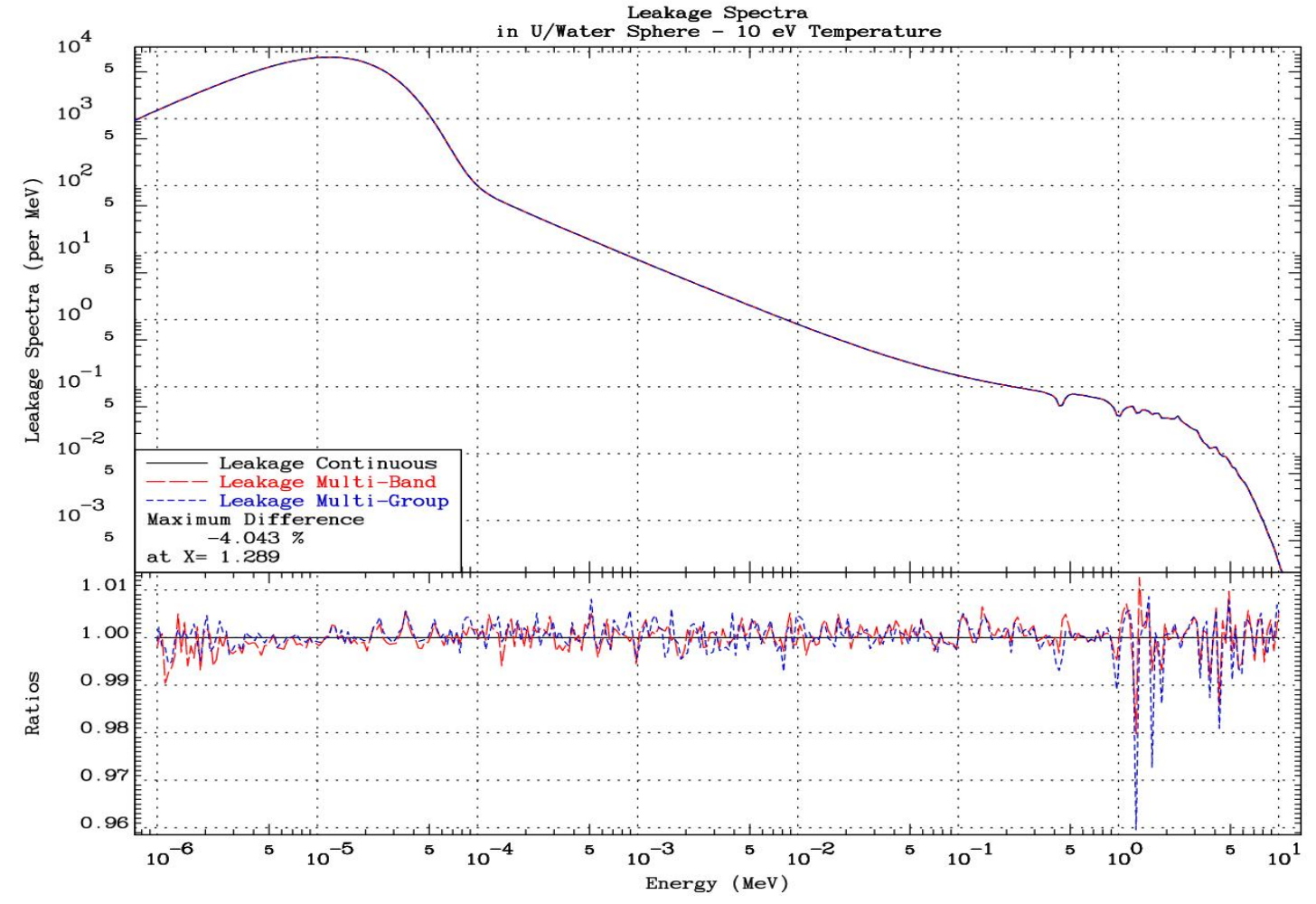

Leakage Spectra 


\section{Comparison of Methods : $100 \mathrm{eV}$ Temperature}

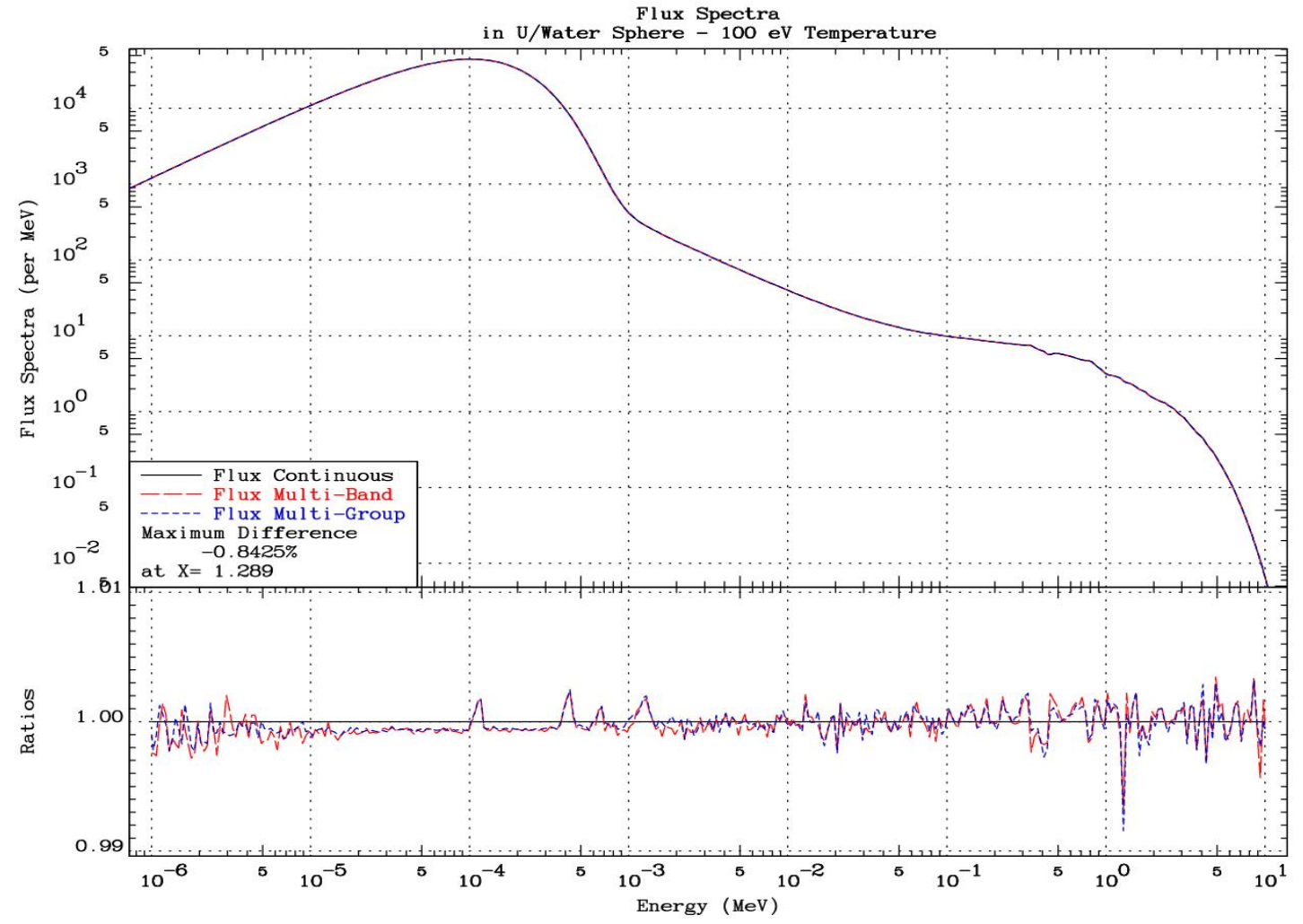

Flux Spectra

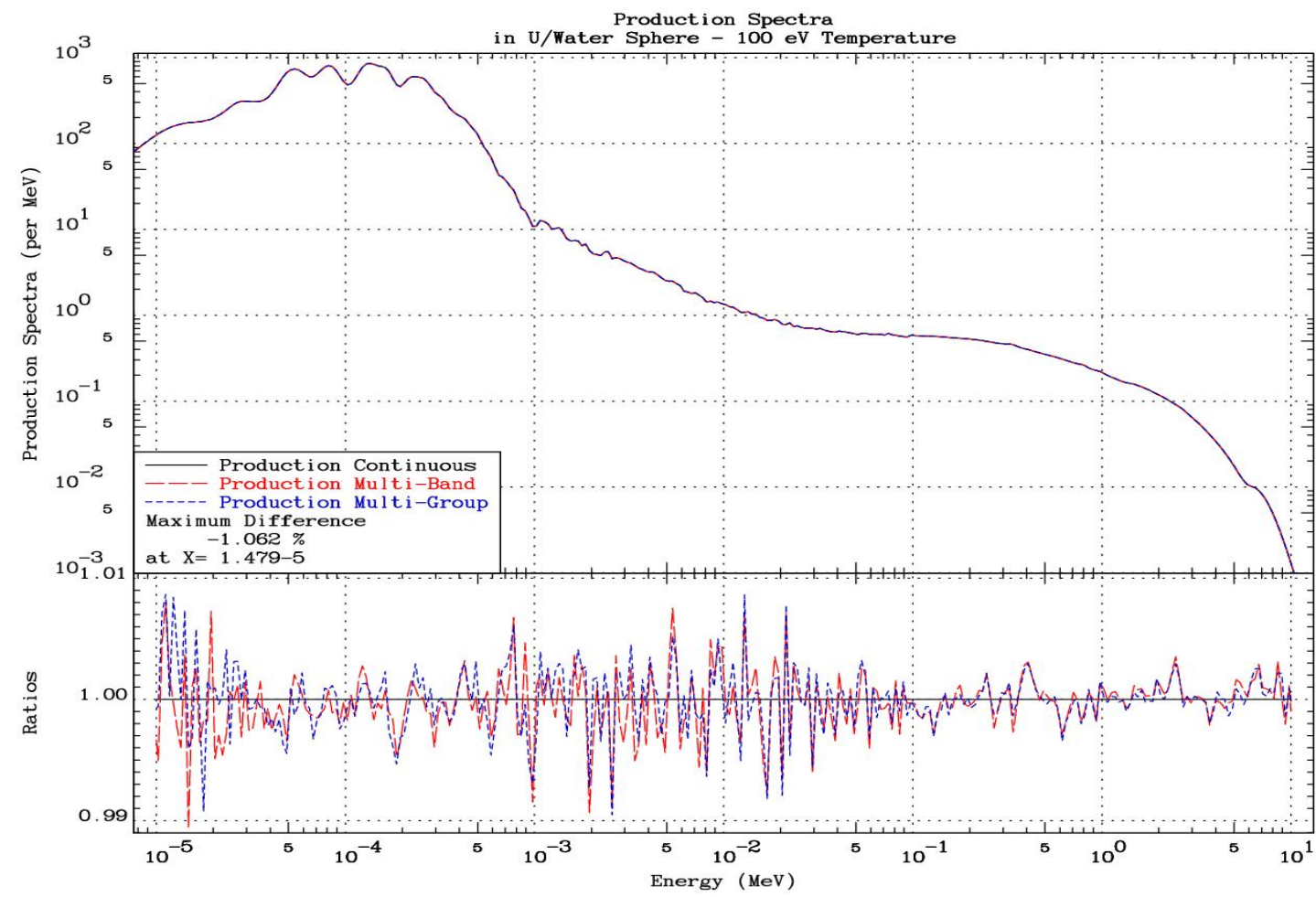

Production Spectra 


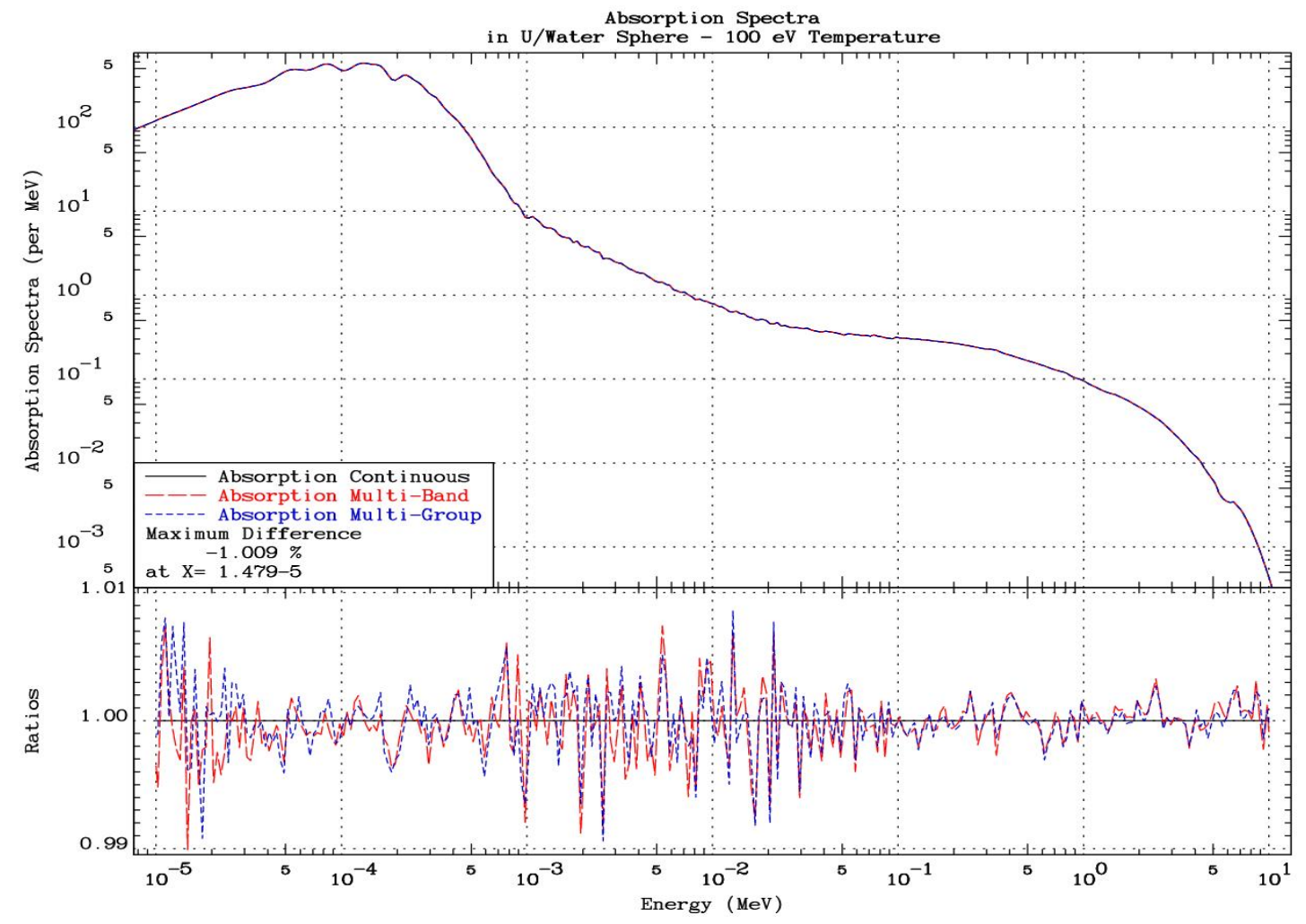

Absorption Spectra

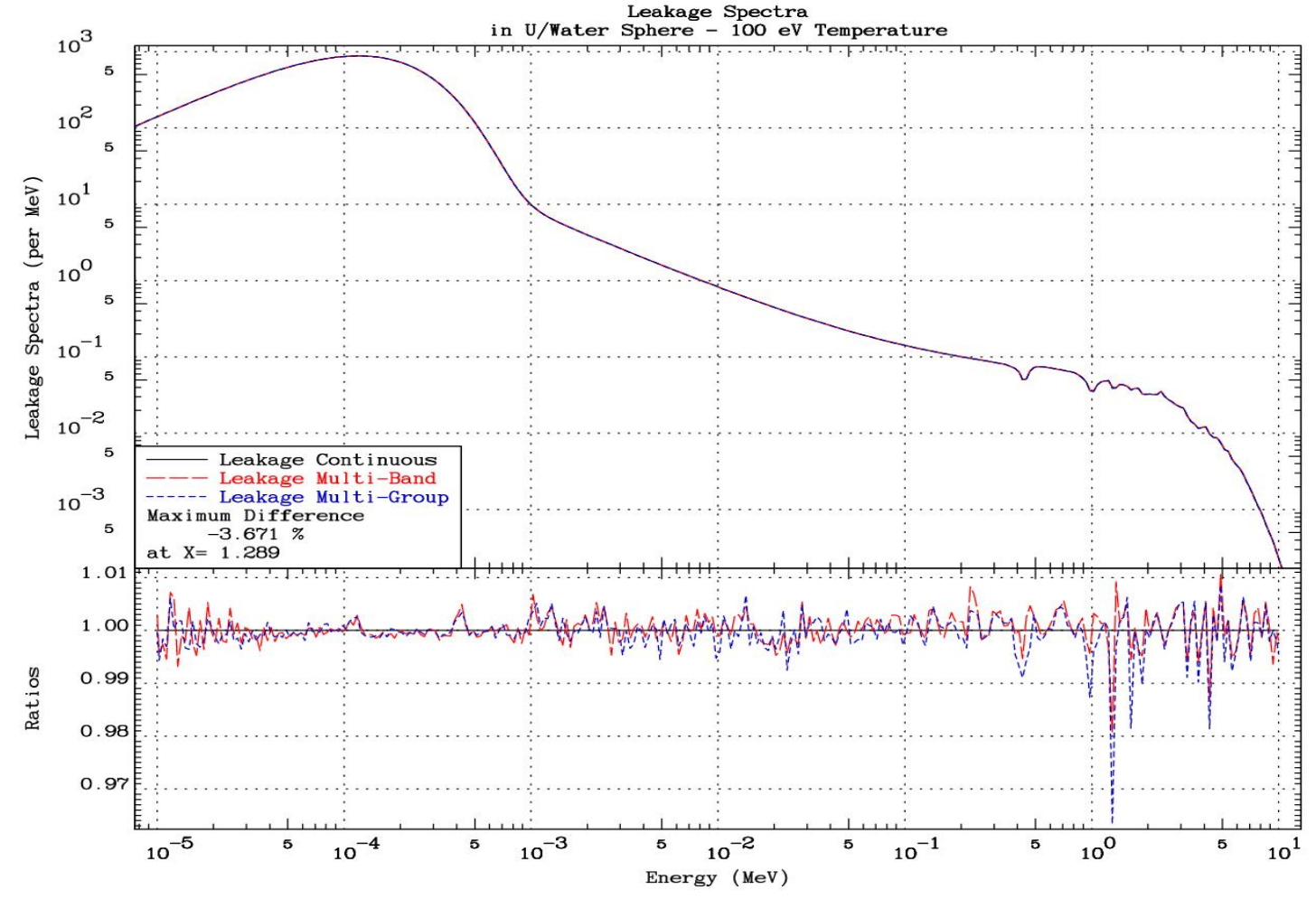

Leakage Spectra 


\section{Comparison of Methods : $1000 \mathrm{eV}$ Temperature}

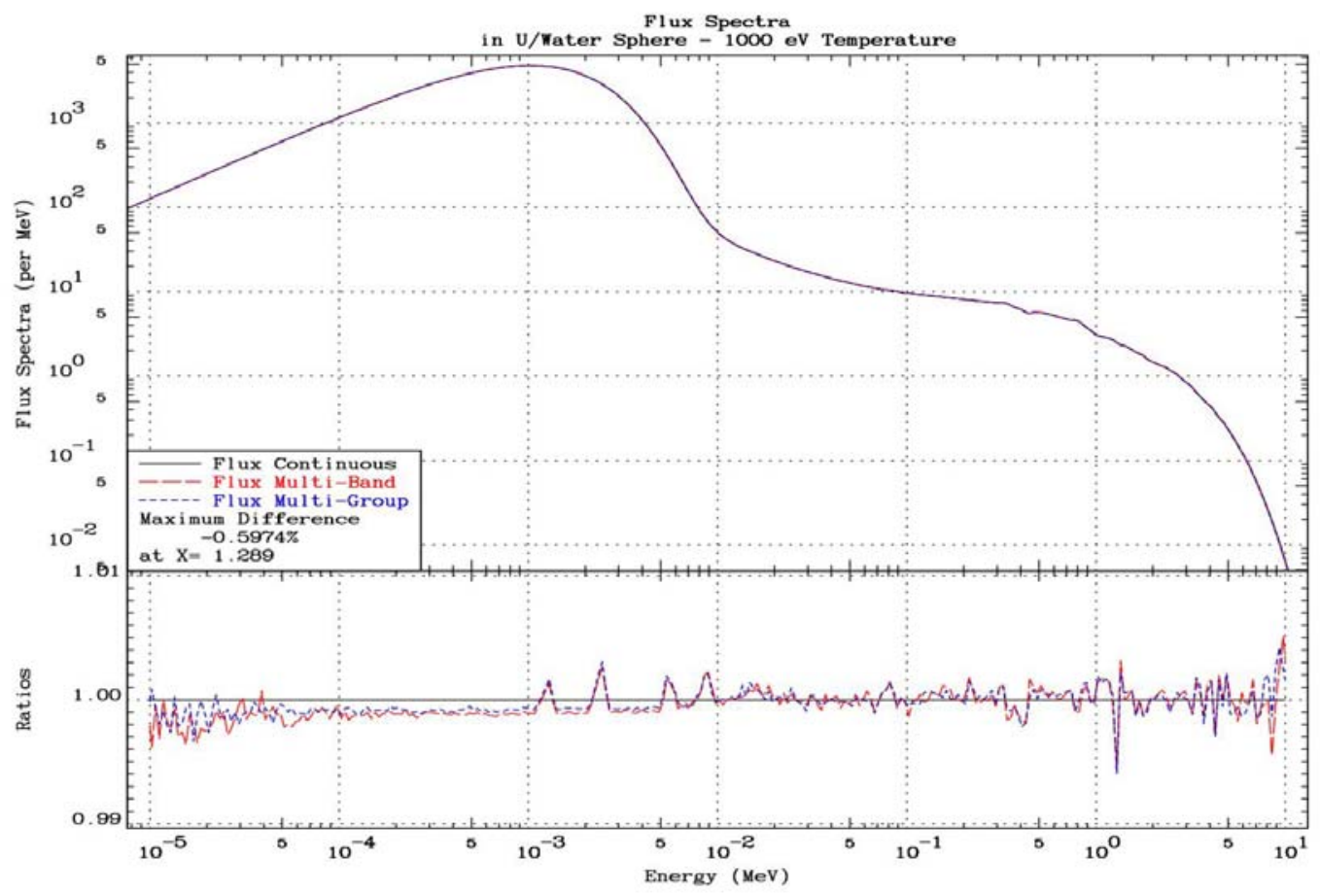

Flux Spectra

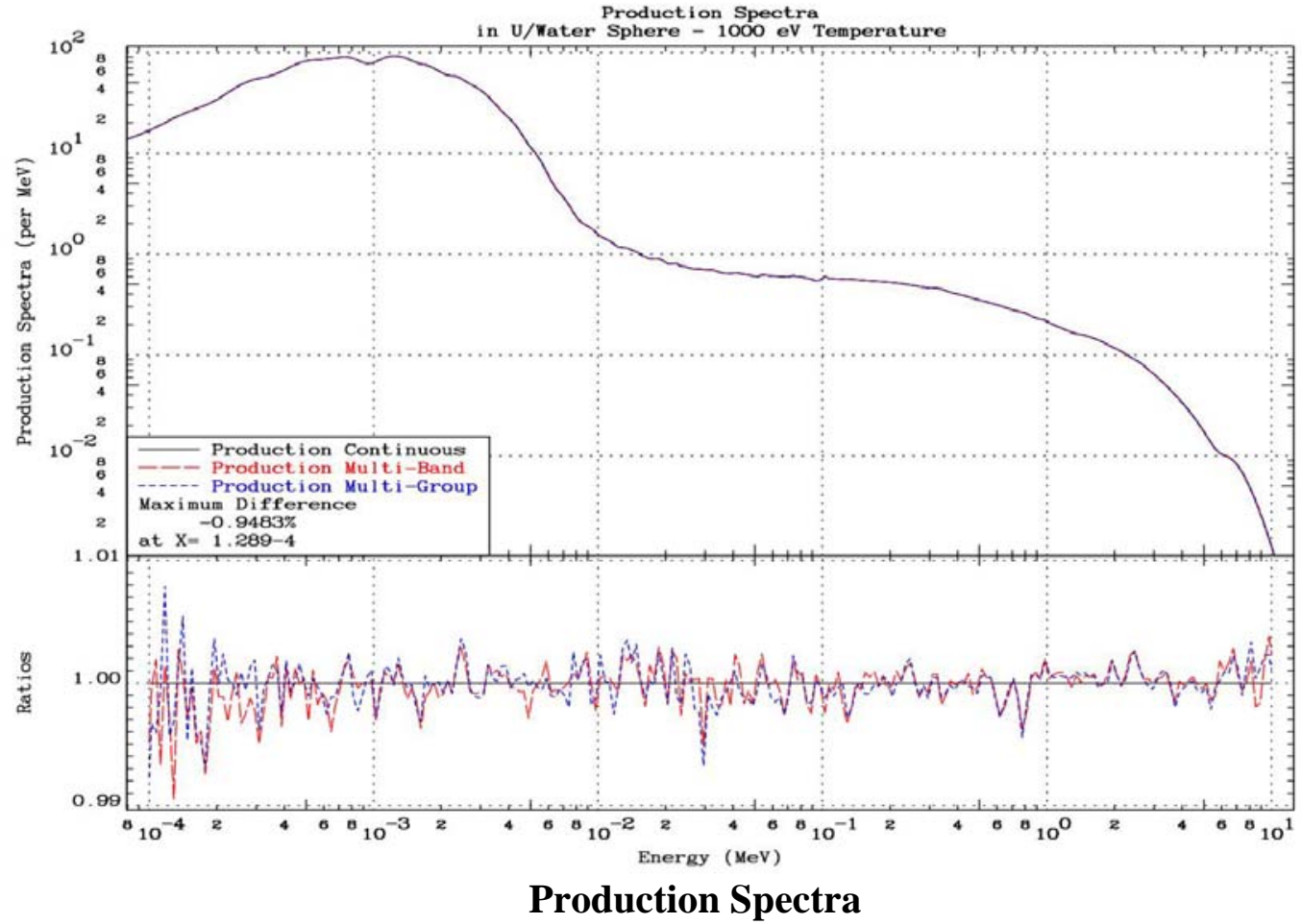




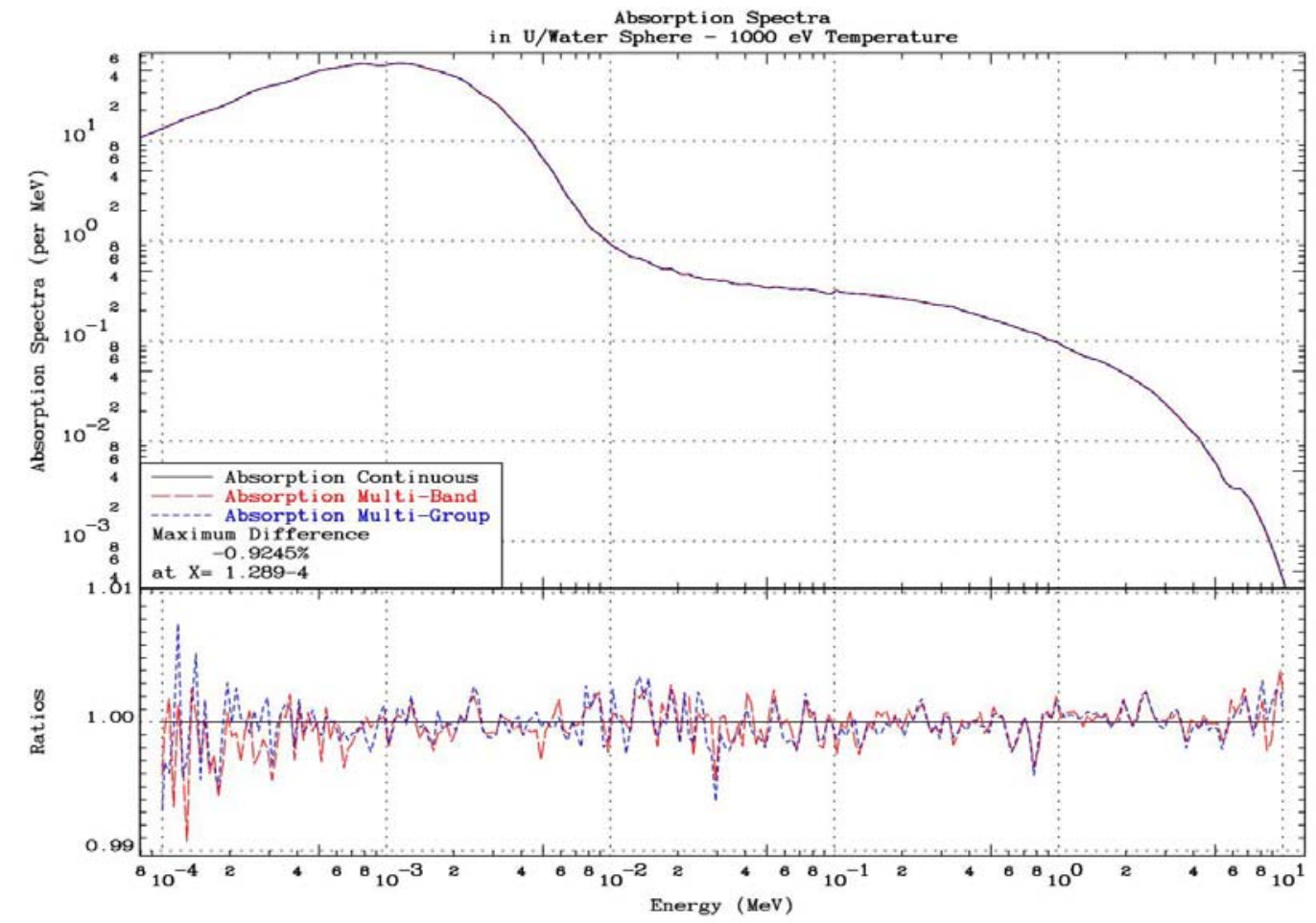

Absorption Spectra

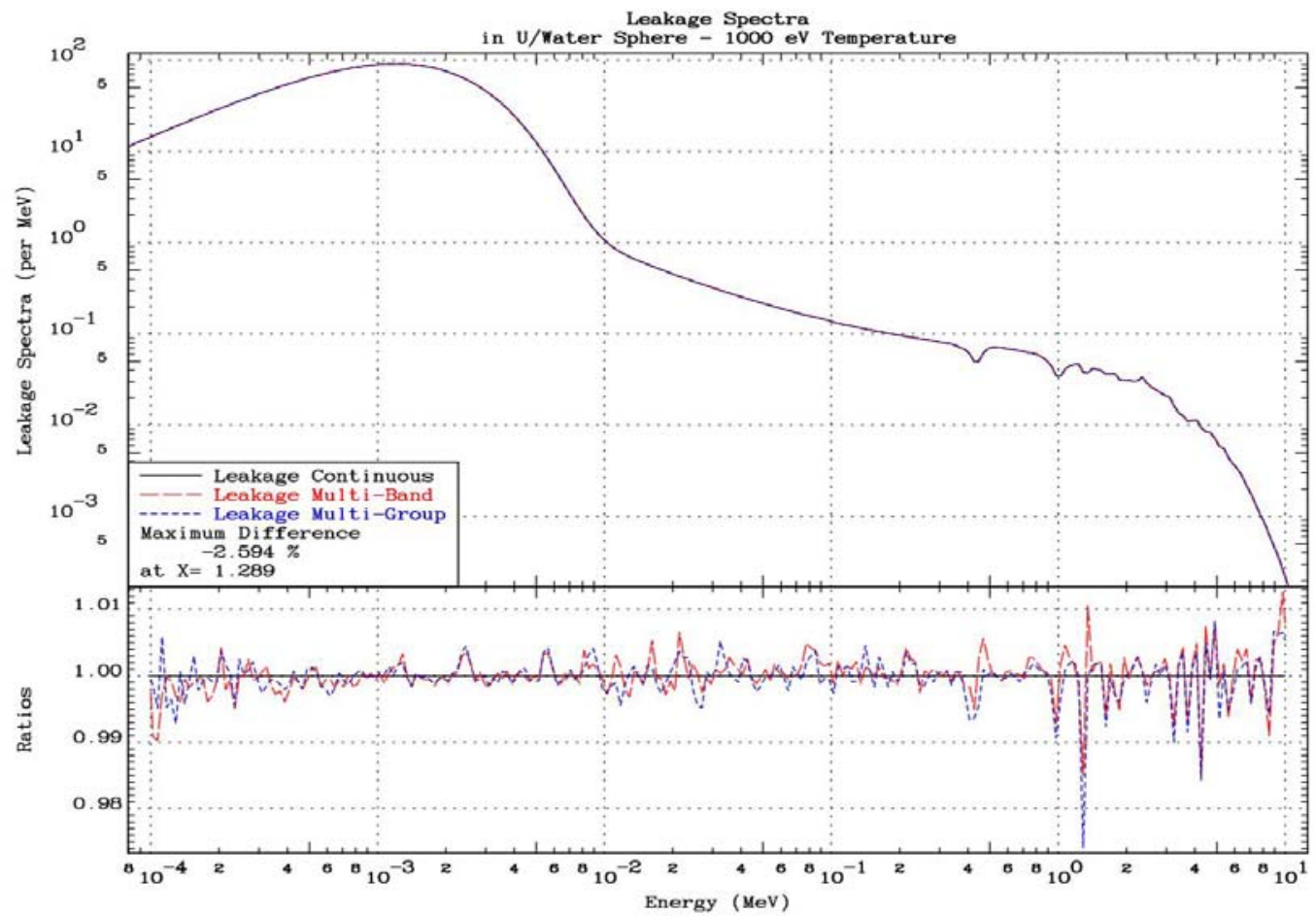

Leakage Spectra 


\section{Conclusions}

Our conclusions are,

1) MERCURY and TART, both using continuous energy cross sections and physical models, are in excellent agreement. In particular the shift of the thermal Maxwellian produced by MERCURY is in excellent agreement with that produced by TART.

2) We still expect large differences for codes using approximate methods, such as multi-group. In particular for a deterministic code, e.g., Sn, even one using 616 groups, but not accounting for self-shielding, can expect large differences from the MERCURY and TART continuous energy results shown here - CAVEAT EMPTOR!!!

\section{References}

[1] MERCURY: User Guide (Version b.8), Lawrence Livermore National Laboratory, Report UCRL-TM-204296, Revision 1, by R. J. Procassini and J. M. Taylor, (2005), more information concerning MERCURY, available online at, http://www.llnl.gov/mercury

[2] TART05: A Coupled Neutron-Photon 3-D, Combinatorial Geometry Time Dependent Monte Carlo Transport Code, UCRL-SM-218009, Lawrence Livermore National Laboratory, by Dermott E. Cullen, November 2005, available online at, http://www.llnl.gov/cullen1/mc.htm

[3] AMTRAN: "Parallel Deterministic Neutron Transport with AMR" by C.J. Clouse, in Computational Methods in Transport, Frank Graziani (Editor), Springer-Verlag, 2006, pp. 499-512.

[4] "How Accurately can we Calculate Thermal Systems”, UCRL-TR-203892, Lawrence Livermore National Laboratory, by Dermott E. Cullen, et al., April 2004, available online at, http://www.llnl.gov/cullen1/pin_cell.htm

[5] "Introduction to Modern Astrophysics", by Bradley W. Carroll and Dale A. Ostlie, Addison Wesley; second edition (2006).

[6] “Mass and Density, Criticality Relationships, Generalized”, UCRL-TR-204988, Lawrence Livermore National Laboratory, by D. E. Cullen., June 2004, available online at, http://www.llnl.gov/cullen1/scaling.htm

[7] “How Accurately Can We Calculate Neutrons Slowing Down In Water?” Lawrence Livermore National Laboratory, UCRL-TR-220605, by D.E. Cullen, et al., April 1, 2006, available online at, http://www.llnl.gov/cullen1/water.htm 
[8] "Application of the Probability Table Method to Multi-Group Calculations," Nucl. Sci. and Eng. 56, p. 387-400, (1974), by D.E. Cullen

[9] "Chapter I: Nuclear Cross Section Processing", by D.E. Cullen, Handbook of Nuclear Reactor Calculation, vol. I, Yigal Ronen (Editor), CRC Press, inc., Boca Raton, Florida (1986).

[10] the TART 2005 nuclear data used in this study is from ENDF/B-VI, Release 8, ENDF-102: Data Formats and Procedures for the Evaluated Nuclear Data File ENDF-6, ENDF-102; BNL-NCS-44945-01/04-Rev., Informal Report, Brookhaven National Laboratory, written by the members of the Cross sections Evaluation Working Group (CSEWG); edited by V. McLane, Revised April 2002.

[11] "Exact Doppler Broadening of Tabulated Cross Sections," Nuclear Science and Engineering 60, p. 199 (1975), by D.E. Cullen and C.R. Weisbin.

[12] SIGMA1 code, part of PREPRO 2004: the 2004 ENDF/B Pre-processing codes (ENDF/B-VII Ready), IAEA-NDS-39, Rev. 12, Nov. 22, 2004, by D.E. Cullen, is available on-line at, http://www-nds.iaea.or.at/ndspub/endf/prepro/

[13] POINT 2004: A Temperature Dependent ENDF/B-VI, Release 8 Cross Section Library”, Lawrence Livermore National Laboratory, UCRL-TR-202284, April 2004, by D.E. Cullen, is available on-line at, http://www.nndc.bnl.gov/point2004/

[14] GROUPIE code, part of PREPRO 2004: the 2004 ENDF/B Pre-processing codes (ENDF/B-VII Ready), IAEA-NDS-39, Rev. 12, Nov. 22, 2004, by D.E. Cullen, is available on-line at, http://www-nds.iaea.or.at/ndspub/endf/prepro/

[15] “Group Constants for Nuclear Reactor Calculations”, Consultant's Bureau, New York (1964) by Bondarenko, I.I., et al.

[16] NJOY: "The NJOY Nuclear Data Processing System, Version 91," Los Alamos National Laboratory report LA-12740-M, by R. E. MacFarlane and D. W. Muir, (October 1994) is still the latest official manual. 


\section{Appendix A: Physics Models still be to implemented in MERCURY}

For the comparison between MERCURY and TART both used exactly the same physics. In order to do this some physics currently available in TART, but not in MERCURY, had to be turned off in TART, this includes,

1) Unresolved resonance self-shielding

2) Delayed neutron spectra

This is work that still has to be done on MERCURY. In order to illustrate the importance of these features below we present TART results for,

1) BEST - with all physics turned on

2) SAME - with the same physics currently available in MERCURY.

The effect on the integral parameters is small, e.g., K-eff changes by roughly $0.1 \%$, even though the effect on differential results is larger. The unresolved resonance regions are in the energy ranges,

1) U235: $2.35 \mathrm{keV}$ to $25 \mathrm{keV}$

2) U238: $10 \mathrm{keV}$ to $149 \mathrm{keV}$

The below figures show an abrupt "jump" in production and absorption in the SAME results relative to the BEST results at the start of the U235 unresolved resonance region at $2.25 \mathrm{keV}$. This is because the BEST results include unresolved region self-shielding which reduces both production and absorption compared to the SAME results. These results demonstrate that,

1) These are important features that must be added to MERCURY

2) Clearly shows that K-eff is not enough to verify true code-to-code agreement; here the BEST and SAME K-eff agree to within roughly $0.1 \%$, and yet there are still important differences in the differential results.
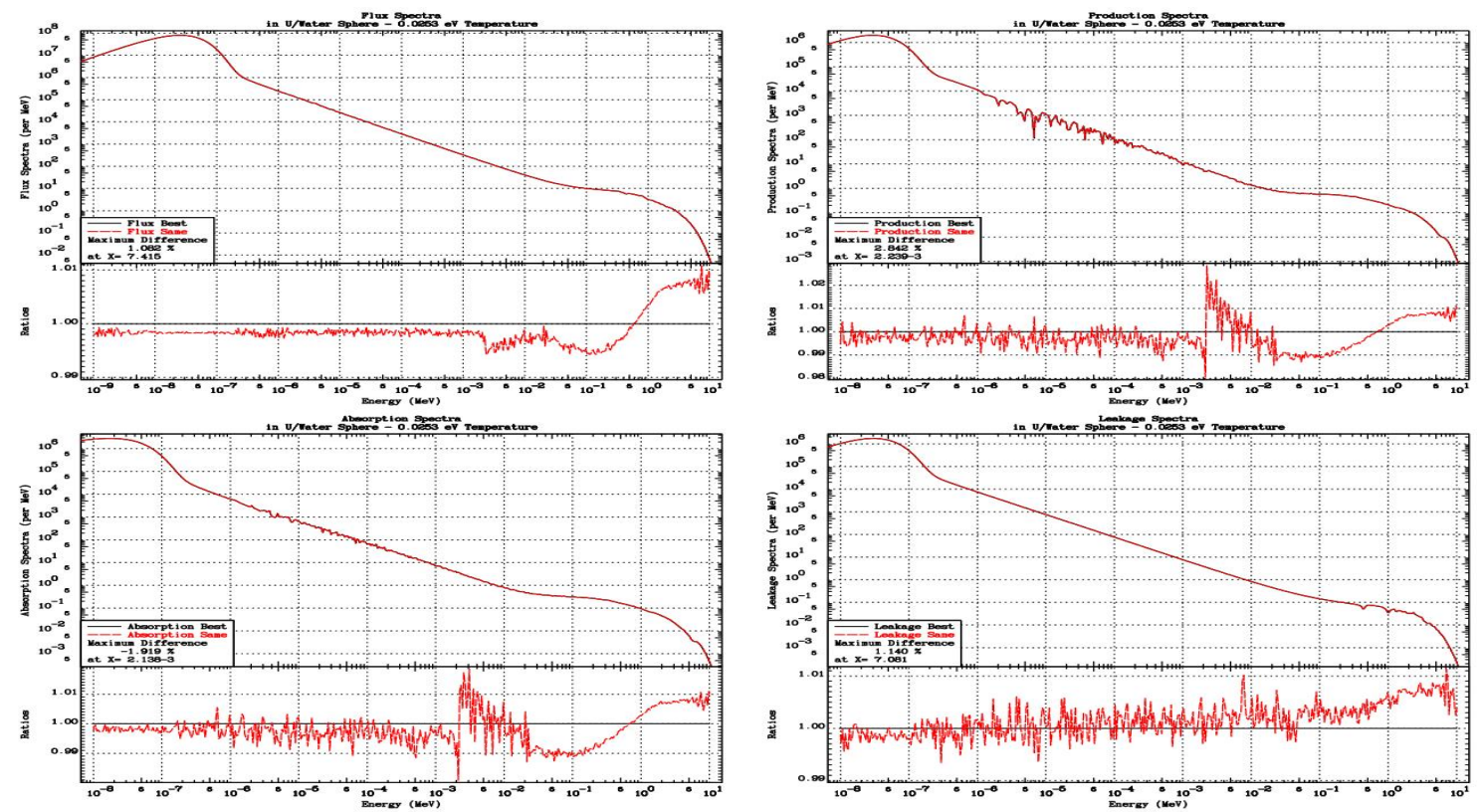


\section{Appendix B: A Discussion of Self-Shielding}

Since the neutron flux at any energy $\mathrm{E}$ is merely the total distance traveled per unit time by all neutrons of energy E, it is not surprising that increasing the cross section, which decreases the average distance that neutrons travel between collisions, leads to a decrease in the neutron flux. This phenomenon is referred to as self-shielding, since in terms of resonances it is the increase in cross section itself which depresses, or shields the resonance, from the flux.

An illustration of self-shielding for a single isolated resonance is shown in the figure below. In the absence of resonances the slowing down flux tends to be $1 / \mathrm{E}$ in shape. When a resonance is present it suppresses the flux (i.e., self-shields it) through the resonance. Below the resonance in energy, for a scattering resonance the flux will return to its $1 / E$ shape and magnitude, whereas for an absorbing resonance the flux will return to its $1 / E$ shape, but with a lower magnitude due to absorption through the resonance.

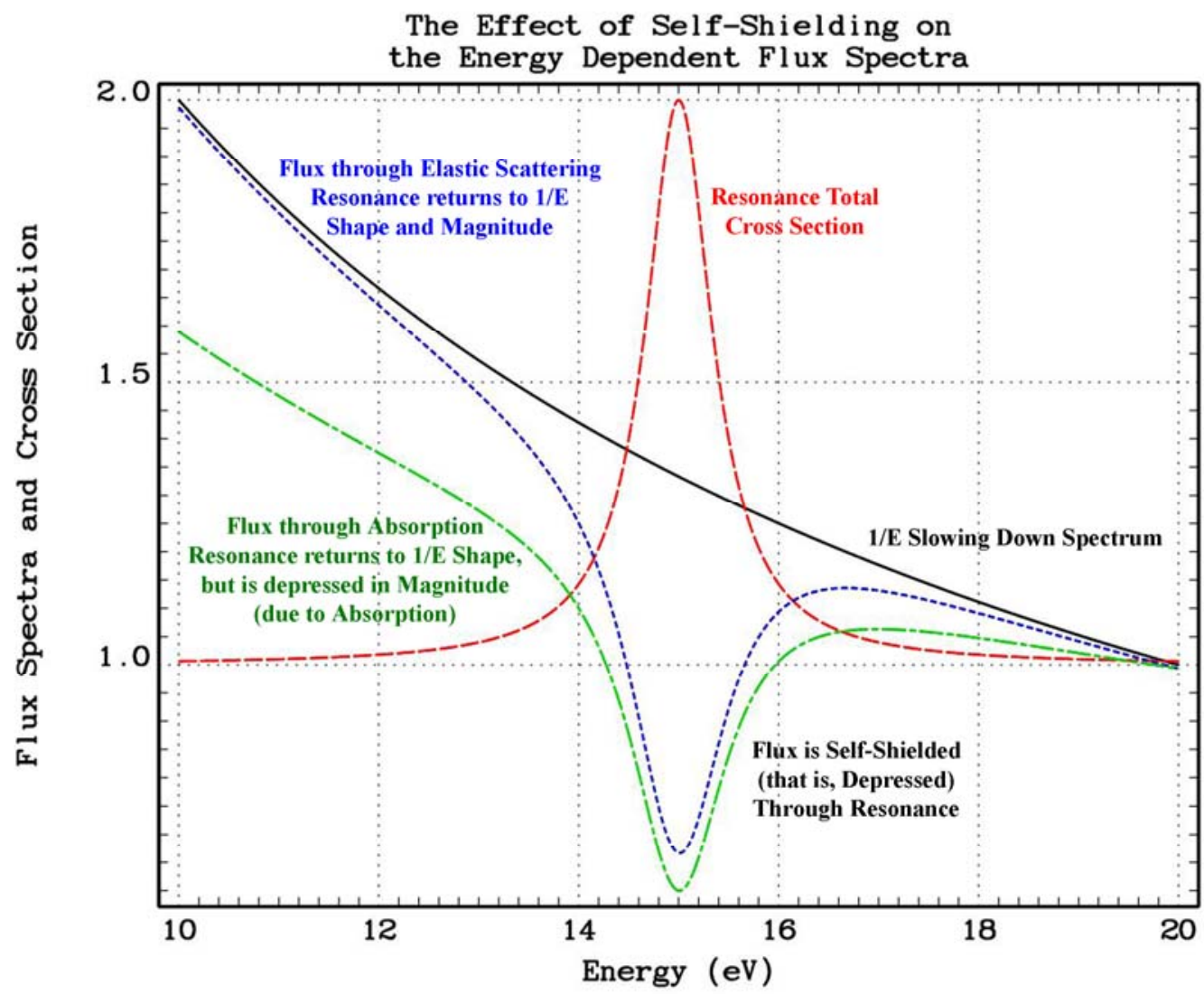


By considering certain limiting cases, it is easy to see the effect that self-shielding has on the neutron flux. First consider a source in a totally absorbing, infinite medium. In this case the Boltzmann equation reduces to,

$\Sigma t(E) * \Phi(E)=S(E)$

Obviously we can immediately solve for the energy dependent flux and we find that it varies inversely to the total cross section,

$\Phi(E)=\frac{S(E)}{\Sigma t(E)}$

Next consider a monoenergetic source in a totally scattering, infinite medium. In this case the Boltzmann equation at energies below source energy reduces to,

$\Sigma t(E) * \Phi(E)=\frac{1}{1-\alpha} \int_{E}^{E / \alpha} \Sigma t\left(E^{\prime}\right) * \Phi\left(E^{\prime}\right) \frac{d E^{\prime}}{E^{\prime}}$

We can immediately solve this equation to find,

$\Phi(E)=\frac{C}{\Sigma t(E) * E}$; where $C$ is a constant that depends on the source strength.

So that in this case we also find that the flux varies inversely as the total cross section. Since the two extremes of total absorption and total scatter both lead to the same simple relationship between flux and total cross section, it is tempting to consider the possibility that this relationship always exists between flux and cross section. Unfortunately, such is not the case and the effects of self-shielding are much more complicated than this.

The simple results shown above are because these two extremes both were for infinite media. In any real problem in a finite media we find that the self-shielding can be dependent not only on energy, but also position, and even direction. This makes modeling self-shielding very difficult. 
In order to see the position dependent effect below let's look at the plot of the flux in the uranium and water. Here we can see an enormous difference between the flux in the two spatial regions of uranium and water, obviously in magnitude, but also look at the energy dependence of the flux in the uranium due to the resonances in uranium. The flux differences in the two spatial regions clearly demonstrate the spatial effects of selfshielding.

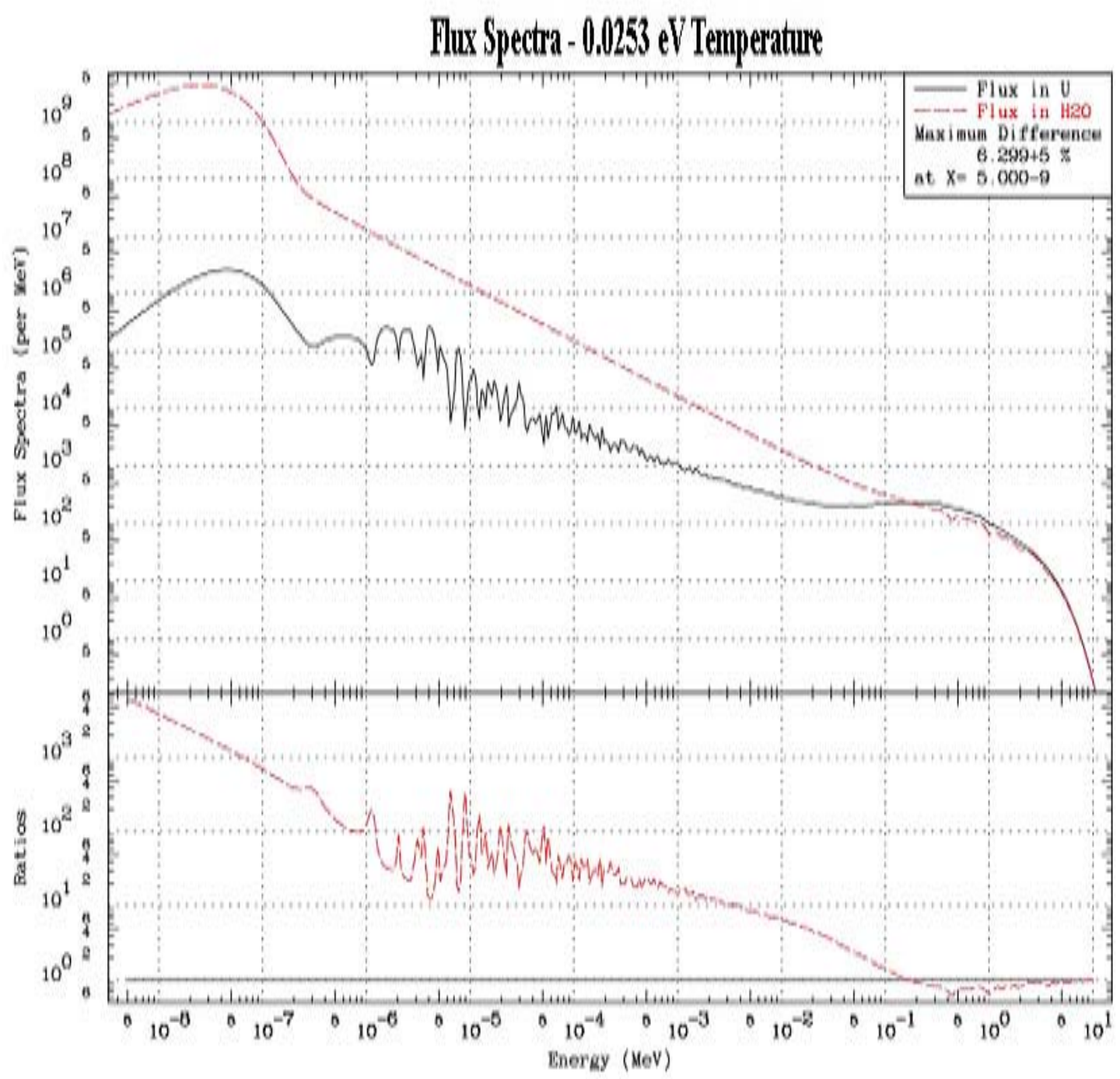


We can also see the effect of direction by below looking at the plot of the current. Note that the Current $\mathrm{U}$ to $\mathrm{H} 2 \mathrm{O}$ and Current $\mathrm{H} 2 \mathrm{O}$ to $\mathrm{U}$ are both defined at exactly the same spatial location; namely the interface between the inner and outer spheres. The only difference is that each is integrated over a different range of directions. At exactly the same spatial point the current directed from the water into the uranium show no selfshielding, whereas the current directed from the uranium to the water shows strong selfshielding, e.g., note the dips in the current near uranium resonances. The current differences in the two direction ranges clearly demonstrate the directional effects of self-shielding.

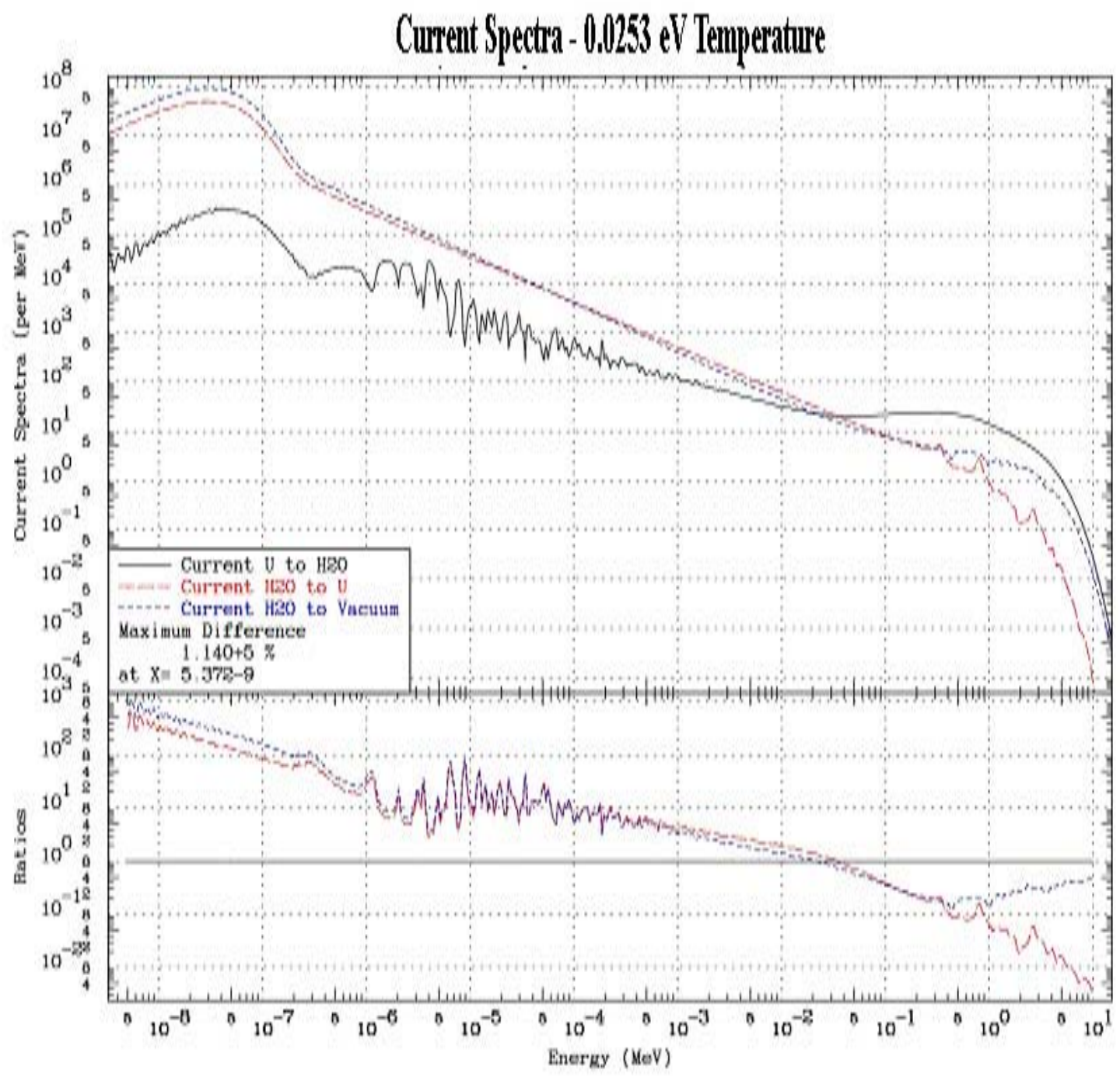


Why do we have to model self-shielding? For our applications using continuous energy cross sections we do not have to model it, but for multi-group applications we do. For multi-group applications before we perform our calculations we must first define our multi-group cross section constants. The multi-group cross section is defined as,

$$
<\Sigma r>=\frac{\int \Sigma r(E) * \Phi(E) d E}{\int \Phi(E) d E}
$$

Where $\operatorname{\Sigma r}(E)$ is the known cross section for some reaction r, and $\Phi(E)$ is our "guess" as to the appropriate flux, or weight, to use. I say "guess" because before running our multigroup calculations we do not know what the flux is; if we knew what it really is we would have our answer and we wouldn't be running neutron transport calculations.

This is how multi-group cross sections are defined, but the situation is much more complicated than indicated by this equation. In general the cross section and flux will be a function of energy (as shown by the above equation), but also by space (both can vary from one location to another), and in the case of the flux, also by direction.

The most widely used "guess" is the Bondarenko approximation [15], where the flux is assumed to be a product of two factors: 1) an energy spectrum, and 2) a total cross section dependent term,

$\Phi(E)=\frac{S(E)}{\Sigma t(E)}$

Here the energy dependent spectrum is fairly smooth: fission spectrum at high energy, 1/E slowing down spectrum, and Maxwellian-like thermal spectrum. It is further assumed that the total cross section for any material can be defined for each constituent of the material by dividing it into the energy dependent total cross section for each constituent plus a constant (energy independent) term to represent all of the other constituents of the material,

$\Phi(E)=\frac{S(E)}{\Sigma t(E)+\Sigma 0}$

Historically this was a very important assumption, because it allowed application independent cross section libraries to be created $[14,16]$. For example, we could define multi-group cross sections for U238 without worrying about what it might actually be mixed with in any application; what it is mixed with presumably could be accounted for by properly defining the constant $\Sigma 0$ to approximate all other constituents.

Primarily what this assumes is that the resonance structure in each isotope is independent of the resonance structure in all other isotopes; this actually works quite well. For example, for our uranium, $75 \%$ U235 and 25\% U238, one would define the U235 cross 
sections assuming some value of $\Sigma 0$ to represent the effect of U238, and we would define the U238 cross sections assuming some other value of $\Sigma 0$ to represent the effect of U235.

Today about the "best" that multi-group codes can do is define Bondarenko self-shielded cross sections, integrated over space, direction, and energy using,

$<\Sigma r>=\frac{\int \Sigma r(E) * \Phi(E) d E}{\int \Phi(E) d E}=\frac{\int \frac{\Sigma r(E) * S(E)}{\Sigma t(E)+\Sigma 0} d E}{\int \frac{S(E)}{\Sigma t(E)+\Sigma 0} d E}$; using various $\Sigma 0$ from small to large

If we consider the difference between unshielded [no $1 / \Sigma t(E)$ weighting] and shielded cross sections [1/ $t(E)$ weighting], it is obvious that the $1 / \Sigma t(E)$ weighting places less weight where the cross section is high, so that generally the shielded cross sections tend to be smaller than the unshielded cross sections.

How important is self-shielding? It is easy to prove that the total cross section monotonically increases as $\Sigma 0$ varies from 0 (totally self-shielded) to infinity (infinitely dilute, or unshielded). What we can easily compare is the totally self-shielded (pure material, $\Sigma 0=0$ ) values to the unshielded values (infinitely dilute, $\Sigma 0=\infty$ ). Below we show the U238 616 group cross sections, unshielded and shielded. Here we need merely look at the ratio to see that in the resonance region below $10 \mathrm{keV}$ there are ENORMOUS differences. We are not talking about differences of a few per-cent; here we see ratios of a factor of 100. This means that when the unshielded cross section is $\mathbf{1 0 0}$ barns, the totally shielded cross section could be less than 1 barn. So to answer the question: yes, self-shielding can be VERY important.

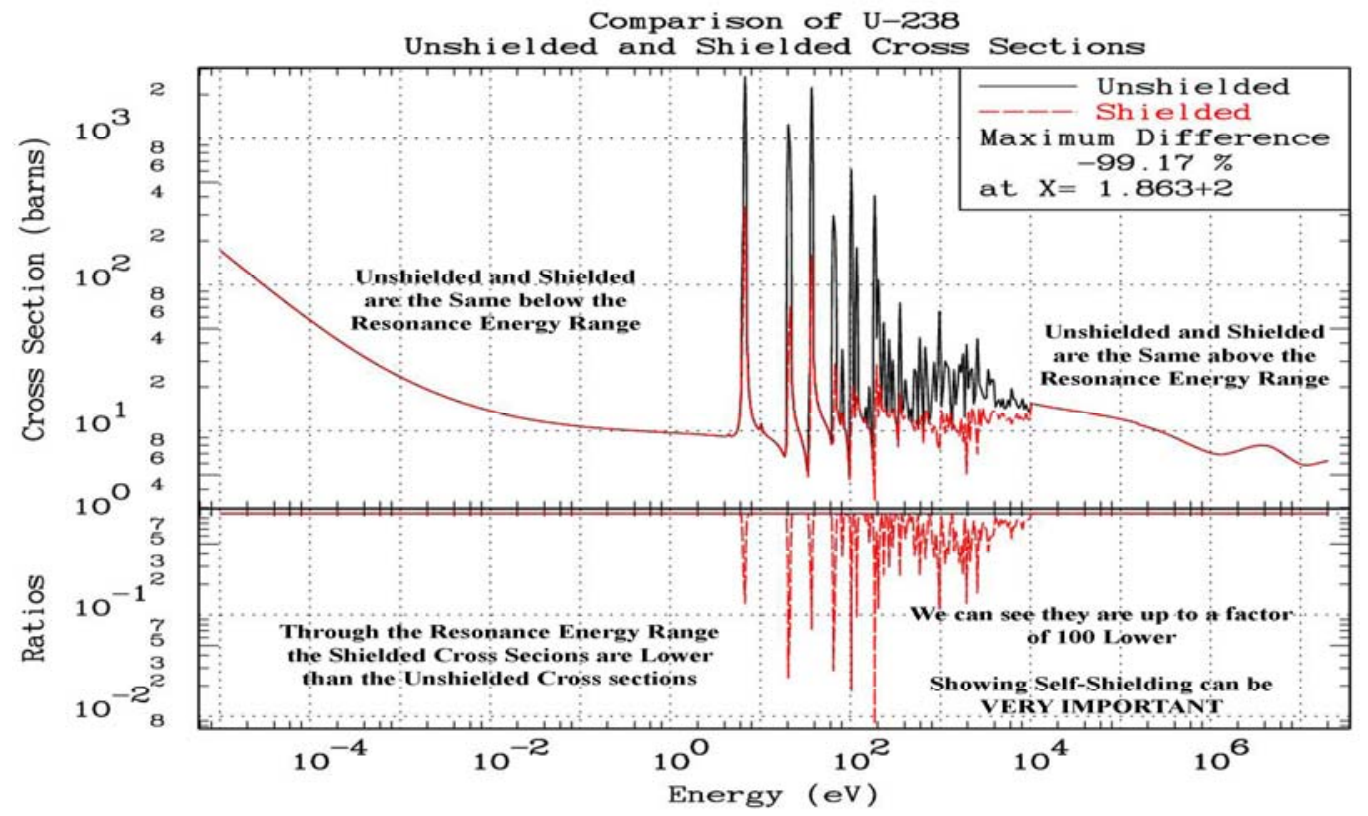


You might think that based on the differences shown above it would be impossible to accurately define multi-group cross sections. Fortunately we find that "Nature is User Friendly", in the sense that if we focus on what's important, which is reactions, not flux, we can see that what Bondarenko's self-shielding model predicts is that the total reaction rate stays about constant regardless of variations in the cross section,

$\Sigma t(E) \Phi(E)=S(E)$

This says that any error we introduce in defining multi-group cross sections will be compensated by a change in the flux to produce about the same total reaction rate. In other words self-shielding may introduce large errors in the flux, but as far as the physically observable reaction rates that we are really interested in we will see much smaller differences. For example, when we calculate K-eff we are only interested in production, absorption and leakage, not simply the flux, so that multi-group estimates of simple integral parameters, such as K-eff, may be much better then one might expect based on the approximations used in this model.

Can we avoid problems of self-shielding by using many groups? This is not really practical. Above we gave an example of U238 cross sections which uses over 67,000 tabulated energy points to represent the energy dependent cross section, and we showed how poor the agreement is between 616 group averages and the continuous energy cross sections. It is unlikely that we will run 67,000 group deterministic (Sn) calculations, and using many fewer groups does not eliminate the problem of self-shielding. Remember that these 67,000 energy points are only for the U238; U235 needs a different set of 28,000 energy points, and so on for additional isotopes in any given problem.

The problem of self-shielding is due to the enormous changes in cross sections over very small energy intervals, e.g., see the above figures showing uranium cross sections. The problem cannot be eliminated by further sub-dividing the energy range into more groups, until the wide of the groups becomes small compared to the width of the resonances, which is not practical.

As we can see the obvious problem with multi-group calculations is that we MUST assume some flux, or weighting, spectrum in order to define our multi-group cross sections before we start our multi-group calculations. Less obvious is what weighting spectrum to use; should it be based on the scalar flux, or scalar current, or something else. With the multi-group method we can only define one cross section in each group, so we are forced to decide between these various possibilities.

The multi-band method $[8,9]$ recognizes the problem that the multi-group method has and instead of further sub-dividing the energy range in the Riemann sense, it further subdivides the total cross section range in the Lebesque sense, thereby directly reducing the range of the total cross section, which is the source of the self-shielding. Unlike the multi-group method which defines averages integrated over space and direction, the multi-band method can approximate the effect of self-shielding as a continuous function of space and direction. With the multi-band method in each group we have more than one 
cross section, and we can define these to simultaneous conserve scalar flux and current. That's why the agreement seen in the comparisons of continuous energy and multi-band is so good. For details concerning the multi-band method, see [9].

Finally let's look in detail at the spatially dependent flux in our uranium/water system. For this purpose we further sub-divided the two spheres into smaller spheres, each of the same volume. The inner uranium sphere is divided into five (5) equal volumes, and these equal volumes are extended out into the water to define five (5) more zones of equal zone of water, and a final zone extending to the outer radius of the outer sphere in used. With equal volumes the uranium zones are not very thick, in terms of radius; see the below list. For example, near the uranium/water interface the first zone inside the uranium (zone \#5) is only $0.55 \mathrm{~cm}$ thick.

$\begin{array}{lrr}\text { Material } & \text { Zone } & \text { Outer Radius }(\mathrm{cm}) \\ \text { uranium } & 1 & 4.456 \\ \text { uranium } & 2 & 5.614 \\ \text { uranium } & 3 & 6.426 \\ \text { uranium } & 4 & 7.073 \\ \text { uranium } & 5 & 7.620 \\ \text { water } & 6 & 8.097 \\ \text { water } & 7 & 8.524 \\ \text { water } & 8 & 8.912 \\ \text { water } & 9 & 9.269 \\ \text { water } & 10 & 9.600 \\ \text { water } & 11 & 15.240\end{array}$

Below the spatially dependent flux is shown for the inner ten (10) zones. The top five curves show the flux in the water, and these are all very similar, i.e., the flux has little spatial dependence. However, as soon as we cross the boundary into the uranium we see the flux being almost exponentially attenuated into the highly absorbing uranium. Near thermal energies the flux in the outer most uranium zone \#5 is almost 2 orders of magnitude (a factor of 100) lower then the flux in the inner most water zone \#6; remember that this is a zone only $0.55 \mathrm{~cm}$ thick and it already differs by this much from the adjacent water zone.. Beyond zone \#5, deeper into the uranium there is essentially no flux. What this means is that in the thermal range all of the uranium burn-up occurs very close to the surface of the uranium. 


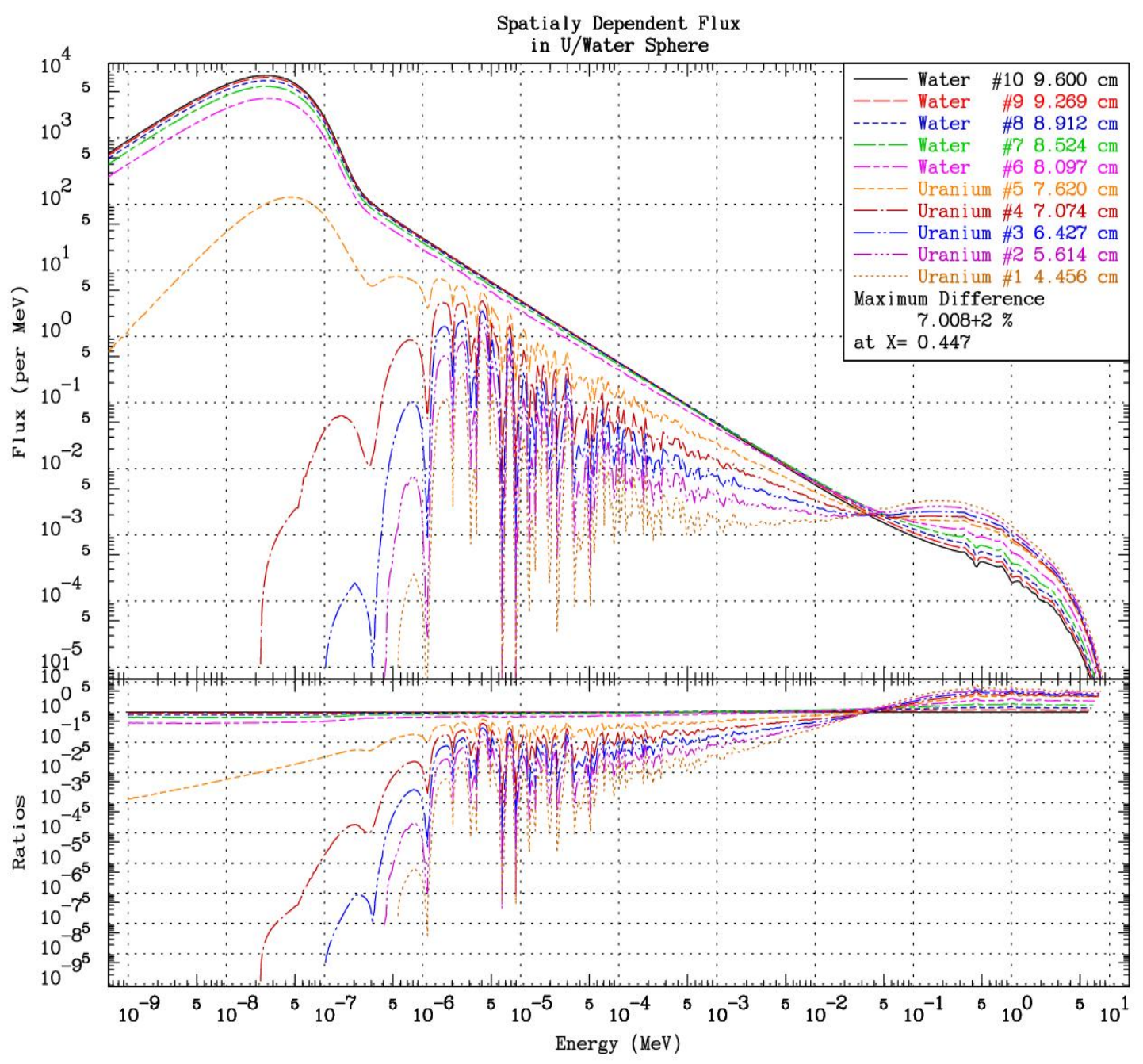


If we look in detail at the flux in the resonance region (the below figure), again we see almost exponential attenuation into the highly absorbing uranium. In this case we can clearly see the effect of the stronger resonances, e.g., note the depression of the flux by a factor of 1 million or more near 6 and $9 \mathrm{eV}$. However, in this case unlike the thermal region where virtually no flux penetrates deep into the uranium, here we see that between resonances the flux is only depressed by factors of 10 to 100 . I say only 10 to 100 , but strong spatially dependent effects over narrow energy ranges such as these are difficult to model with deterministic codes.

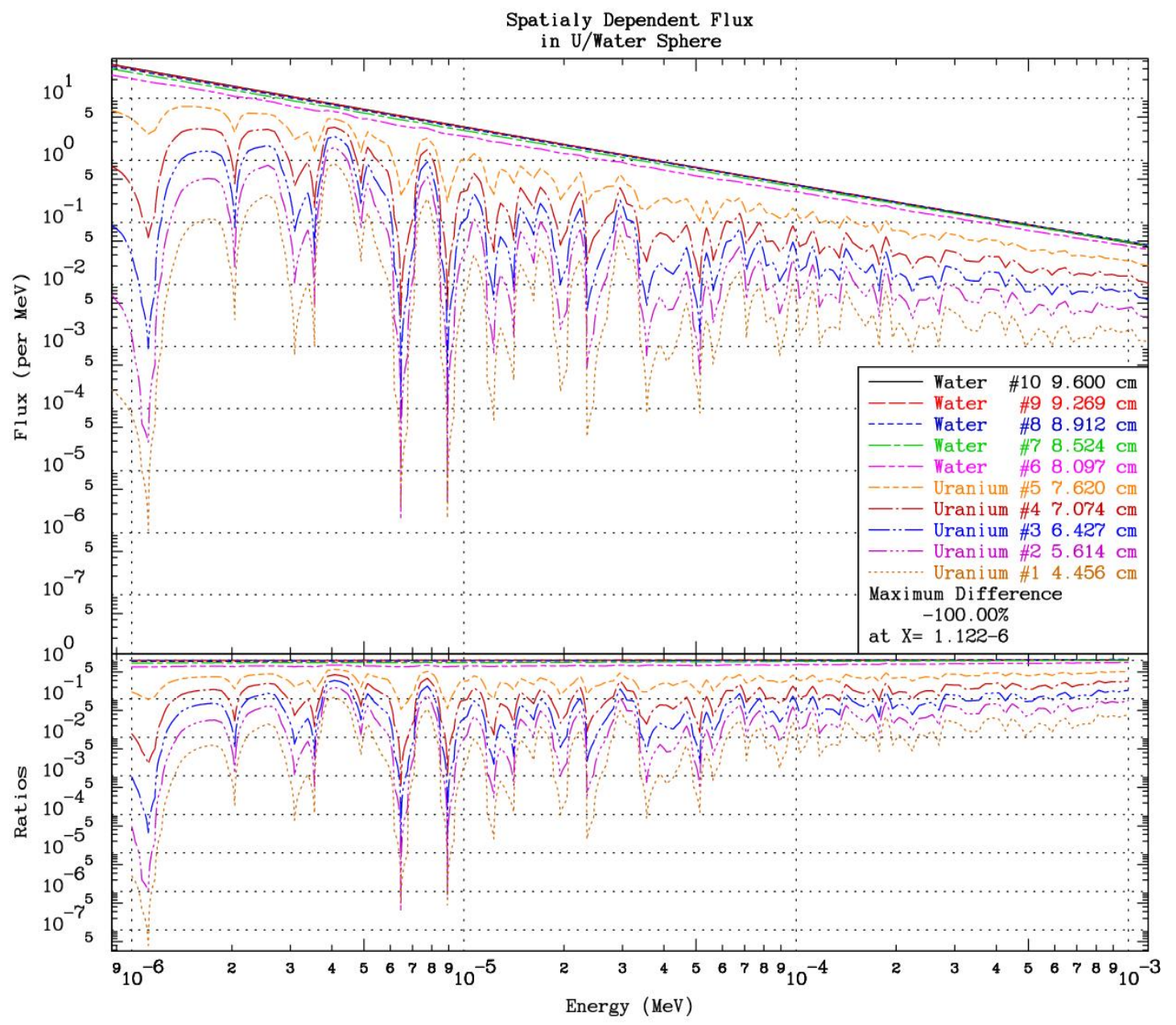


Just a reminder, that multi-group cross sections are defined by,

$<\Sigma r>=\frac{\int \Sigma r(E)^{*} \Phi(E) d E}{\int \Phi(E) d E}$

To expect accurate results we must model $\Phi(E)$ as accurately as possible, however in reality $\Phi(E)$ is also a function of space and direction. In principle to model our uranium/water system we can see from the above plot of the spatially dependent flux, $\Phi(E)$, we should use different fluxes in many thin layers of uranium. From the above figures I hope the reader can appreciate how difficult it would be to accurately model this system using multi-group calculations. Within the uniform uranium inner sphere the flux is very strongly spatially dependent self-shielded. Near the uranium/water interface there is little self-shielding, but deeper into the uranium the flux is quickly heavily selfshielded. Within the scope of Bondarenko self-shielding [15] it is assumed that within any given material, such as our uranium sphere, one value of $\Sigma 0$ can be used to define the "average" cross sections to use throughout the material. Hopefully the above results demonstrate how difficult this would be. In contrast the multi-band method can reproduce this spatially and directionally dependent self-shielding of the flux. 


\section{Appendix C: the Definition of K-eff}

For time independent codes there is a very simple textbook definition that can be used to define K-eff. It is the ratio of the number of neutrons produced by fission in one generation to the number produced in the preceding generation; these codes do not consider anything else. For time dependent codes or codes that define K-eff in terms of a balance between neutrons produced and removed this is more complicated, because fission is not the only process that can produce neutrons; there is also $(n, 2 n),(n, 3 n)$, etc., and how codes handle these lead to different definitions of K-eff. Below I'll explain the differences.

Starting from the time dependent, linear Boltzmann equation in general geometry,

$$
\frac{1}{v} \frac{\partial N}{\partial t}+\Omega * \nabla N+\Sigma t^{*} N=\iint(<v>\Sigma f+\Sigma s c a t t e r+2 \Sigma n, 2 n+3 \Sigma n, 3 n+\ldots \ldots .) N d \Omega^{\prime} d E^{\prime}
$$

Where $N(r, \Omega, E, t)$ is the neutron flux, $v^{*} n(r, \Omega, E, t), v$ is the neutron speed, $\Sigma t$ is the macroscopic total cross section, $\langle v\rangle$ is the average number of neutrons emitted per fission, $\Sigma f$, $\Sigma$ scatter , $\Sigma n, 2 n, \Sigma n, 3 n$, etc., are the macroscopic cross sections for each type of event. For simplicity I will use neutron density $n(r, \Omega, E, t)$ in the following,

Integrate over all space, energy, and direction

$$
\frac{\partial n}{\partial t}+\left[L^{*} v^{*} n\right]+\left[\Sigma t^{*} v^{*} n\right]=\left[(<v>\Sigma f+\Sigma s c a t t e r+2 \Sigma n, 2 n+3 \Sigma n, 3 n+\ldots . .) v^{*} n\right]
$$

Collecting terms together we find a simple equation defining the time dependent behavior of the system,

$$
\begin{aligned}
& \frac{\partial n}{\partial t}=\alpha^{*} n \\
& \alpha=[(<v>\Sigma f+\Sigma s c a t t e r+2 \Sigma n, 2 n+3 \Sigma n, 3 n+\ldots . .) v]-\left[L^{*} v\right]-\left[\Sigma t^{*} v\right] \\
& =[\text { Production rate }]-[\text { Removal Rate }]: \quad \text { Removal }=\text { Leakage }+ \text { Absorption }
\end{aligned}
$$

The time constant $(\alpha)$ is a physical observable and as such has a unique value that we can determine. The non-uniqueless of K-eff and related terms is because exactly the same terms appear in this definition of $\alpha$ as positive and negative terms that we can completely cancel (scatter), or as simply related terms that we can partially cancel (n,2n).

I will divide the total cross section by events according to how many neutrons result from each type of event: none - capture, (n,p), (n,a), etc., one - scatter, (n,np), (n,na), etc., more than one - fission, $(n, 2 n),(n, 3 n)$, etc.. All of those events that result in one neutron 
do not directly effect the neutron balance of the system (they effect it indirectly through the leakage), and appear in exactly the same form in this definition as positive and negative terms, so that we can cancel them. Upon cancelling scatter, (n,np), (n,na), etc.,

$\alpha=[(<v>\Sigma f+2 \Sigma n, 2 n+3 \Sigma n, 3 n+\ldots .) v]-.\left[L^{*} v\right]-[(\Sigma n, 0+\Sigma f+\Sigma n, 2 n+\Sigma n, 3 n+\ldots) * v]$

Up to this point all or least most of the codes use the same definitions, and this is the definition that TART uses, i.e., any event that introduces additional neutrons into the system is considered production, and any event that produces neutrons also removes neutrons, etc., $(\mathrm{n}, 2 \mathrm{n})$ removes one neutron and produces two neutrons,

Production rate $=[(<v>\Sigma f+2 \Sigma n, 2 n+3 \Sigma n, 3 n+\ldots .) v$.

Removal Rate $=$ Leakage + Absorption $=\left[L^{*} v\right]+[(\Sigma n, 0+\Sigma f+\Sigma n, 2 n+\Sigma n, 3 n+\ldots) * v]$

Other codes change this to agree with the 1940's - '50 textbook definition of K-eff where production is only due to fission. This requires them to subtract $2 \Sigma n, 2 n+3 \Sigma n, 3 n+$.. from the production and removal resulting in the definitions,

Production rate $=[(<v>\Sigma f) v]$

Removal Rate $=\left[L^{*} v\right]+[(\Sigma n, 0+\Sigma f-\Sigma n, 2 n-2 \Sigma n, 3 n-3 \Sigma n, 4 n-\ldots) * v]$

Note, that we still have exactly the same definition of the physically observable time constant $(\alpha)$, and for an exactly critical system K-eff remains unity using either of these definitions. Regardless of how they define production and removal, the codes define,

$\alpha=[$ Production Rate $]-[$ Removal Rate $]=\left[\frac{\text { Pr oduction }}{\text { Re moval }}-1\right] *[$ Removal Rate $]$

$$
=[\text { K-eff }-1] / \operatorname{Tr} \quad \operatorname{Tr}=\text { Removal Time }=1 /[\text { Removal Rate }]
$$

K-eff $=$ Production $/$ Removal $=$ Production $/[$ Absorption + Leakage $]$

Here we can see that even though the time constant $(\alpha)$ has a unque definition, K-eff and the removal time, do not, since all codes do not define production and removal the same way. With the TART definition any event that produces more than one neutron ends a generation, and adds to the removal, $\Sigma f+\Sigma n, 2 n+\Sigma n, 3 n+\ldots$ and also adds to the production $<v>\Sigma f+2 \Sigma n, 2 n+3 \Sigma n, 3 n+\ldots \ldots$. Codes that do not consider that $(n, 2 n),(n, 3 n)$, etc., end a generation, add nothing to production for these events and subtract from the removal, $\Sigma n, 2 n+2 \Sigma n, 3 n+3 \Sigma n, 4 n+\ldots$. Let me repeat this: these events are treated as NEGATIVE removal, which physically makes no sense at all. This is just an old outdated convention, a mathematical trick that has somehow survived in a surprising number of today's neutron transport codes.

In order to illustrate how silly this older definition is in practice, below I present a portion of a TART output file, which defines K-eff, removal and production using TART more 
generally definition and the old definition where only fission is assumed to produce neutrons. Compare the upper portion of this table, using TART's definitions, to the lower portion. The lower portion treats $(n, 2 n)$ and $(n, 3 n)$ as NEGATIVE removal; this reduces removal from 1.0 to 0.9955 , almost $0.5 \%$. It also reduces the production from 0.96877 to 0.96430 , again almost $0.5 \%$, showing that in this case $(n, 2 n)$ produces almost $0.5 \%$ of the neutrons in this system. These differences are not at all insignificant, and yet many codes continue to define production based on the 1940's and '50 textbook definition that neutron production can only occur due to fission.

Again, for systems that are exactly critical K-eff is exactly the same using either definition. However, for systems that are not exactly critical we do see differences in Keff. For example, in the below table the two different definitions result in 0.9687 versus 0.9686 , a difference of 0.0001 or $0.01 \%$; in this case quite small.

But consider an accelerator driven system where say beryllium is used to multiple the accelerator source of fast neutrons. What is the multiplication or production of neutrons for this system? According to the old definition where only fission can produce neutrons, there is no production or multiplication - there is only NEGATIVE absorption, which is complete nonsense.

The bottom line is that if we are interested in the actual population of neutrons within any system, there is no question that reactions other than fission, such as (n,2n), produce significant number of neutrons that define the actual neutron population, and as such TART includes them as neutron production. The alternative is to treat $(n, 2 n)$, etc., as NEGATIVE absorption, which is a cute mathematical trick to obtain a neutron balance, but which makes no physical sense.

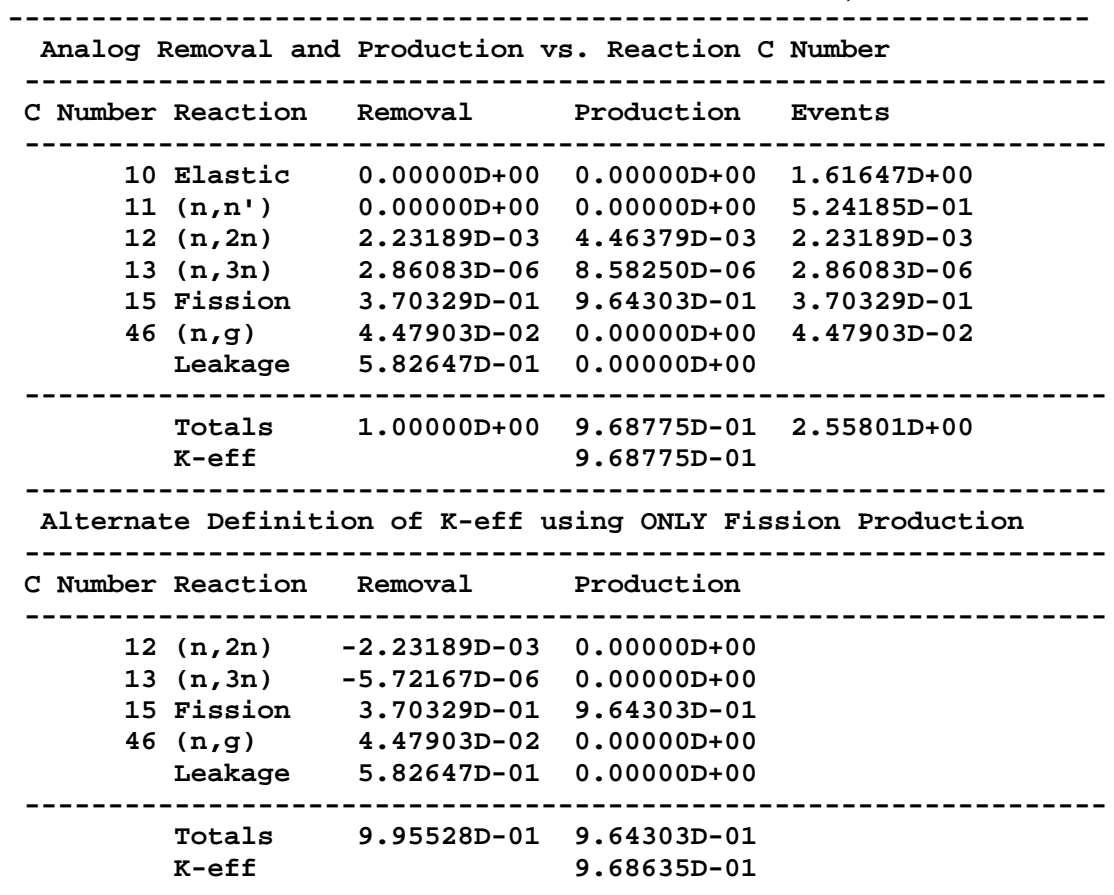

